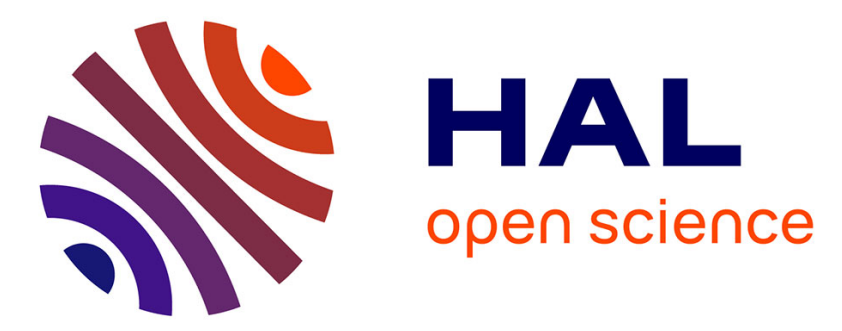

\title{
The Scf/Kit pathway implements self-organized epithelial patterning
}

Alexandre Chuyen, Charlotte Rulquin, Fabrice Daian, Virginie Thomé, Raphaël Clément, Laurent Kodjabachian, Andrea Pasini

\section{To cite this version:}

Alexandre Chuyen, Charlotte Rulquin, Fabrice Daian, Virginie Thomé, Raphaël Clément, et al.. The Scf/Kit pathway implements self-organized epithelial patterning. Developmental Cell, 2021, 56 (6), pp.795-810.e7. 10.1016/j.devcel.2021.02.026 . hal-03216498

\section{HAL Id: hal-03216498 https://hal-amu.archives-ouvertes.fr/hal-03216498}

Submitted on 4 May 2021

HAL is a multi-disciplinary open access archive for the deposit and dissemination of scientific research documents, whether they are published or not. The documents may come from teaching and research institutions in France or abroad, or from public or private research centers.
L'archive ouverte pluridisciplinaire HAL, est destinée au dépôt et à la diffusion de documents scientifiques de niveau recherche, publiés ou non, émanant des établissements d'enseignement et de recherche français ou étrangers, des laboratoires publics ou privés. 
1 The Scf/Kit pathway implements self-organised epithelial patterning.

2

3 Alexandre Chuyen ${ }^{1}$, Charlotte Rulquin ${ }^{1}$, Fabrice Daian $^{1}$, Virginie Thomé ${ }^{1}$, Raphaël Clément ${ }^{1}$,

$4 \quad$ Laurent Kodjabachian ${ }^{1,2,3^{*}}$ and Andrea Pasini ${ }^{1,3 *}$

5

6 1. Aix-Marseille Univ, CNRS, IBDM, Marseille, France

7

8 * Correspondence : andrea.pasini@univ-amu.fr; laurent.kodjabachian@univ-amu.fr

9

$10 \quad 2$. Lead contact

11 3. Co-senior authors

\section{Highlights}

14 - Immature multiciliated cells transit from a disordered to an ordered pattern before intercalating

15 into the epidermis outer layer.

16 - The transition is a self-organising process based on mutual repulsion and affinity for the outer-

17 layer cell membranes.

18 - Arp2/3-dependent actin remodelling is required for pattern emergence

19 - The Scf/Kit pathway promotes both repulsion- and affinity-mediated movements

\section{eTOC blurb}

22 In developing Xenopus epidermis, immature multiciliated cells (MCCs), initially randomly

23 distributed within an inner layer, emerge in an orderly pattern among cells of the outer layer. This process involves MCC mutual repulsion and affinity towards outer-layer intercellular junctions. 
2 How individual cell behaviours lead to the emergence of global patterns is poorly 3 understood. In the Xenopus embryonic epidermis, multiciliated cells (MCCs) are born in a 4 random pattern within an inner mesenchymal layer, and subsequently intercalate at regular 5 intervals into an outer epithelial layer. Using both experiments and mathematical modelling, 6 we show that this transition from random to ordered distribution relies on mutual repulsion 7 among motile immature MCCs, and affinity towards outer-layer intercellular junctions. 8 Consistently, Arp2/3-mediated actin remodelling is required for MCC pattern emergence. 9 Using multiple functional approaches, we show that the Kit tyrosine kinase receptor, 10 expressed in MCCs, and its ligand Scf, expressed in outer-layer cells, are both required for regular MCC distribution. Membrane-associated Scf behaves as a potent adhesive cue for

12 MCCs, while its soluble form promotes their mutual repulsion. On the other hand, Kit 13 expression is sufficient to confer order to a disordered heterologous cell population. Our 14 work reveals how a single signalling system can implement self-organised large-scale 15 patterning.

17 Keywords:

$18 \mathrm{Scf} /$ Kit, pattern formation, self-organisation, Xenopus, ciliated epithelium, cell motility, cell-cell 19 repulsion, adhesion, actin cytoskeleton, contact inhibition of locomotion. 
Orderly cellular patterns have always captured the attention of scientists, and understanding the

3

4 mechanisms and forces that control their establishment is one of the long-standing aims of developmental biology. In the past decades, classical and molecular genetics approaches have led to the identification of robust Gene Regulatory Networks (GRNs) that generate patterns by concomitantly specifying cellular identity and position within developing embryos (Briscoe and Small, 2015; Davidson, 2010). However, patterns of regular cell distribution can also emerge when the stochastic movements of motile cell populations are partially restricted, for example by mutual repulsion. Such phenomena have been described in rat retina (Galli-Resta et al., 2002), mouse cerebral cortex (Villar-Cervino et al., 2013), zebrafish epidermal pigmented cells (Walderich et al., 2016) and Drosophila haemocytes (Davis et al., 2012) but the underlying molecular mechanisms are only starting to be unravelled.

In vertebrates, one striking example of ordered cellular pattern is provided by the embryonic epidermis of the amphibian Xenopus, where hundreds of cells carrying multiple motile cilia (Multiciliated Cells or MCCs) are found distributed among mucus-producing goblet cells, according to a strict spacing pattern (Deblandre et al., 1999). The ontogeny of this mucociliary epithelium occurs through a multistep process (Cibois, 2014). At cleavage stages, asymmetric cell divisions partition the embryonic non-neural ectoderm into an outer epithelial and an inner mesenchymal layer. At gastrula stages, the outer layer gives rise to a sealed epithelium containing only goblet cells, while MCCs are born within the inner layer, together with osmoregulatory ionocytes, serotonin-secreting Small Secretory Cells (SSCs) and basal cells. The Notch, Bmp and Wnt pathways have been shown to control the number of MCCs, ionocytes and SSCs born within the inner layer (Deblandre et al., 1999) (Stubbs et al., 2006) (Hayes et al., 2007) (Quigley et al., 2011) (Cibois et al., 2015; Haas et al., 2019; Huang and Niehrs, 2014). Starting at neurula stages, the MCCs, ionocytes and SSCs migrate apically in separate waves to radially intercalate into the outer layer, where they will complete their differentiation (Cibois, 2014). Strikingly, however, 
only MCCs display a regular pattern of distribution in the mature epithelium. Although the precise physiological function of Xenopus MCCs remains to be addressed, a regular pattern of distribution may be optimal to homogenize fluid flows powered by beating cilia. MCCs always insert into the outer layer individually and exclusively in correspondence of junctions among at least three outerlayer goblet cells (vertices) (Chung et al., 2014; Deblandre et al., 1999; Stubbs et al., 2006). Radial intercalation involves a first phase in which immature MCCs in the inner layer emit apically-polarized protrusions to probe outer-layer vertices, followed by a phase of apical expansion, when their apical aspect actually emerges at the surface of the outer layer and progressively expands in its plane to allow for cilia assembly (Chung et al., 2014; Sedzinski et al., 2016; Stubbs et al., 2006). While multiple studies have addressed the cellular and molecular mechanisms of radial MCC intercalation (Chung et al., 2014; Kim et al., 2012; Sedzinski et al., 2016, 2017; Sirour et al., 2011; Werner et al., 2014), how the regularly dispersed pattern of mature MCCs is generated remains to be resolved. It has been suggested that MCC pattern formation may result from an interplay between the mechanisms that define the numbers of committed immature MCCs and the number and distribution of outer-layer vertices available for intercalation (Deblandre et al., 1999; Stubbs et al., 2006; Werner and Mitchell, 2012). While these two parameters are indisputably involved, it is unclear whether they are sufficient to explain pattern emergence. Here, we hypothesize that MCC movement may be another key ingredient, which has never been evaluated. Using transient transgenesis, we could record the movements of immature MCCs in the inner layer, and concluded that MCC spacing pattern was largely established prior to intercalation. Using mathematical modelling, we found that mutual repulsion among MCCs and affinity for outer-layer intercellular junctions are sufficient to account for the final dispersed pattern. These two behaviours were readily apparent from our live recordings. Confirming the importance of MCC movements, inhibition of Arp2/3-mediated actin remodelling impaired lamellipodia-like protrusions in MCCs, homotypic MCC repulsion and MCC spacing pattern, without affecting intercalation. We also uncover a hitherto unknown role of the signalling 
pathway dependent on the interaction between the transmembrane tyrosine kinase receptor Kit, expressed in MCCs, and its ligand Scf, expressed in outer-layer cells, for MCC pattern emergence. The Scf/Kit signalling pathway has a well-established role in controlling actin cytoskeleton and cell motility in a variety of vertebrate systems (Lennartsson and Ronnstrand, 2012; Meininger et al., 1992; Runyan et al., 2006; Samayawardhena et al., 2007; Wehrle-Haller et al., 2001; Zuo et al., 2016) and Kit stimulation with Scf was found to result in the phosphorylation and activation of ARP2/3 (Mani et al., 2009). Using a variety of functional assays, we established the capacity of the Scf/Kit system to promote both the repulsive and adhesive interactions predicted in silico, and thus identified for the first time a major regulator of the Xenopus MCC patterning process. Remarkably, we found that a soluble form of the Scf ligand promotes MCC dispersion, while a membrane-bound form mediates adhesion. This work illustrates how a large cell population can self-organise to adopt a regular spacing pattern, through semi-random movements constrained by a balance between repulsive and attractive cues, both transduced by the same ligand-receptor system.

\section{RESULTS}

\section{Emergence of MCC ordered pattern precedes radial intercalation.}

Fluorescent In Situ Hybridization (FISH) of Xenopus embryos with the MCC marker $\alpha$-tubulin ( $\alpha$ tub) revealed that at stage 14 (early neural plate stage), immature MCCs were irregularly distributed within the non-neural ectoderm inner layer (Figure 1A) and very often in direct contact with each other (Figure 1B). Co-staining with an antibody against the tight junction protein ZO-1, to mark the outer-layer apical tight junctions, also showed that at this stage $\alpha$-tub-positive MCCs were not positioned in correspondence of outer-layer vertices (Figure 1A, 1C). In the course of development, however, $\alpha$-tub-positive MCCs were increasingly often found as separate cells (Figure 1A, 1B), and progressively localized each in correspondence of an individual vertex (Figure 1A, 1C), into which they finally inserted, thus progressively taking up an apparently more 
orderly pattern (Figure 1A). To quantify the progression from a randomly dispersed to a regular distribution, we developed an order index based on Delaunay triangulation of the centroids of $\alpha$ tub-positive cells. Such an index is inversely proportional to the variation of distances among centroids and would reach its highest value when MCC centroids are perfectly ordered in staggered rows. As shown in Figure 1D, the order index of the MCC population progressively increased over the 12 hours elapsed between stage 14 and stage 25, when MCCs are intercalated into the outer layer.

The apparent increase in order may result non-exclusively from selective death or cell fate reversal of misplaced MCCs, or from MCC movements. Fluorescent detection of the apoptotic executioner Caspase 3/7 activity as a proxy of apoptosis failed to reveal dying cells in the ectoderm of control embryos from stage 15 to stage 18, as opposed to cycloheximide-treated embryos (Trindade et al., 2003) (Figure S1A and movie S1). To evaluate the possible contribution of cell-fate reversal, we performed $\alpha$-tub FISH in embryos from a transgenic line expressing RFP under the control of the MCC-specific $\alpha$-tub promoter (Stubbs et al., 2006). Due to the long RFP half-life (26h), any immature MCC switching its identity to that of a different inner-layer cell type should retain RFP fluorescence while losing endogenous $\alpha$-tub transcripts. Such cases were never observed, ruling out MCC fate reversal during normal development (Figure S1B, S1C). In contrast, we noticed that robust $\alpha$-tub signal was occasionally detected in RFP-negative cells, compatible with rare events of asynchronous MCC specification (Figure S1B, S1C).

The lack of MCC death or fate reversal led us to favour the hypothesis that cell motility might play a major role in the progressive emergence of a regular pattern of MCC distribution.

\section{Time-lapse video microscopy reveals MCC motility}

To evaluate whether MCCs are motile, we injected embryos with constructs carrying the fluorescent markers GFP or LifeActGFP (Belin et al., 2014) under the control of the $\alpha$-tub promoter, and followed the behaviour of labelled MCCs in living embryos. Despite the high 
degree of mosaicism, which restricted transgene expression to a small percentage of MCCs, fluorescence levels were sufficient to allow live recording starting from stage 14 and for a duration of up to 8 hours. Spinning-disk confocal video microscopy showed that GFP-expressing MCCs indeed moved actively within the plane of the inner layer until they eventually stopped in correspondence of vertices where they began to intercalate (Figure 2A and movie 1). As the Factin-binding reporter LifeActGFP allowed for much better visualisation of the whole cell shape and of cellular protrusions, we exploited it for most of our subsequent experiments. We were thus able to record the mean instant speed of 55 LifeActGFP-expressing MCCs over a maximum of 8 hours. As shown in Figure 2B, MCCs increased their mean instant speed to about $0.5 \mu \mathrm{m} /$ minute during the first 2.5 hours, then progressively slowed down and stopped in the course of the successive 6 hours. We also found that LifeActGFP-expressing MCCs extended and retracted actin-rich lamellipodia-like processes within the ectoderm inner layer and progressively changed their shape from flat mesenchymal to more compact and pyramidal (Figure $2 \mathrm{C}$ and movie 2). These observations suggested that cell motility may indeed be an important factor in the establishment of a regular MCC pattern.

\section{A mathematical model for motile MCC pattern establishment.}

We next built an energy-based mathematical model to identify the minimal ingredients necessary to recapitulate the observed self-organisation of motile MCCs. In this simplified model, MCCs can move in a bi-dimensional plane against a network representing outer-layer junctions as defined by ZO-1 immunofluorescence (see STAR methods). As homotypic repulsion is often required for homogeneous dispersion of motile cellular populations (Davis et al., 2012; VillarCervino et al., 2013), we introduced a first term related to MCC mutual repulsion, such that proximity to another MCC increased energy (see STAR methods). In addition, we reasoned that homing of MCCs to outer-layer vertices must imply a marked affinity for these epithelial junctions. Thus, we introduced a second term related to affinity, such that the overlap of MCCs 
with junctions decreased energy (see STAR methods). Since in our two-dimensional model vertices represent the regions of highest possible overlap with junctions, no additional vertexspecific information was needed. The model also included a steric constraint in the form of a hard-core potential, to prevent complete overlap between MCCs. Starting from a random spatial distribution of MCCs, at a density comparable to typical situations observed at stage 14, a standard Monte-Carlo scheme was used to allow system evolution. At each step, any given MCC was assigned a random, diffusion-like prospective displacement. A displacement was systematically accepted if it lowered the system total energy. If on the contrary it increased system energy, its acceptance probability decayed exponentially with energy increase (see STAR methods). This eventually led the system towards equilibrium configurations. In an affinitydominated regime (i.e. when the contribution of mutual repulsion is negligible), MCCs rapidly relocated at nearby vertices, where they could maximise their interaction with outer-layer junctions and minimize their energy. However, this occurred regardless of the position of other MCCs, so that no overall regularity of distribution was observed (Figure 2D and movie 3 ). In contrast, in a repulsion-dominated regime, MCCs repelled each other and distributed according to a regular pattern that maximized their mutual distance, regardless of the position of outer-layer vertices (Figure 2E and movie 4). Finally, in an intermediate regime in which the repulsion and affinity terms were commensurate, a trade-off situation was observed: MCCs were located at outer-layer vertices and individually scattered, although not in a perfectly regular pattern (Figure $2 \mathrm{~F}$ and movie 5; see also STAR methods). This last condition closely resembled the distribution normally found in embryos. Thus, the self-organised MCC distribution can be captured in a simple mathematical model where cell movements are required, but constrained by an interplay of homotypic repulsion and affinity for outer-layer junctions.

Mutual repulsion and affinity for outer-layer junctions underlie MCC pattern establishment. 
We next explored whether MCC mutual repulsion and affinity for outer-layer junctions predicted to be important in our in silico model were indeed detectable in vivo. Firstly, in cases where low mosaicism allowed for visualisation of several neighbouring LifeActGFP-expressing MCCs we found that, whenever in the course of their movements two such cells came into contact, they withdrew from each other (Figure 3A, A' and S2A and movie 6). Measurement of the instant speed of pairs of colliding MCCs showed that, independently of the developmental stage at which the collision took place, it was quickly followed by a decrease in the speed of both cells (Figure 3B). Colliding MCCs came to a halt, remained in contact for 12.11+/-18.96 minutes (average +/SD) (Figure 3G), then regained speed in opposite directions in over $80 \%$ of observed cases (Figure 3A-B). Secondly, imaging of embryos simultaneously injected with $\alpha$-tub::LifeActGFP and with a synthetic mRNA coding for a membrane-localized form of RFP to label outer-layer cells revealed that, while in the inner layer, most MCCs preferentially moved along the junctions among outerlayer cells $(92.55 \%$ of 94 analysed MCCs closely followed outer-layer junctions over $>2 \mathrm{hrs}$ ) (Figure 3C, $\mathrm{H}$ and movie 7). In contrast, only 25\% of these MCCs still appeared to migrate along junctions when the junction-labelling RFP signal was rotated $90^{\circ}$ anticlockwise in silico to provide a control (Figure $3 \mathrm{H}$ ). These observations confirmed that homotypic repulsion and affinity for outer-layer junctions are likely important for the emergence of a regular MCC pattern.

The actin cytoskeleton appeared to be involved in both MCC mutual repulsion and in their affinity for outer-layer junctions. MCCs often established transient contacts with each other via LifeActGFP-enriched lamellipodia-like structures that are subsequently retracted (Figure 3A, A', S2A and movie S2) and LifeActGFP-rich cellular protrusions were apically extended by MCCs as they crawled along junctions among outer-layer cells (Figure S2B and movie S3). As a first step towards dissecting the role of the actin cytoskeleton in MCC patterning, we took advantage of well-characterised pharmacological inhibitors of the two main pathways of actin polymerisation: CK-666, an inhibitor of the actin-branching nucleator Arp2/3 (Hetrick et al., 2013) and SMIFH2, inhibitor of the actin-bundling factor Formin (Rizvi et al., 2009). FISH for $\alpha$-tub coupled with 
ZO-1 IF on embryos treated with $100 \mu \mathrm{M}$ CK-666 from stage 12 and fixed at stage 25 showed that most MCCs had properly emerged at the outer-layer apical surface (Figure S2C), but were irregularly distributed and often in direct contact with one another (Figure 3E, 3I, 3J). Consistent with this observation, spinning-disk confocal video microscopy of $\alpha$-tub::LifeActGFP- and mRFP-injected embryos treated with CK-666 revealed that MCCs displayed a decrease of their mean instant speed (Figure S2D), but a four-fold increase of their mutual contact time (average time of contact +/- SD: 48.54+/-49.10 min) (Figure 3G), thus forming small clusters (Figure S2E and movie S4). Furthermore, the correlation between collision and variation in speed was lost (Figure S2F). However, in CK-666-treated embryos, MCCs still moved preferentially along outerlayer junctions (92.31\% of 26 analysed MCCs) (Figure 3H). Consistent with decreased MCC instant speed, the total distance covered by tracked MCCs and their total displacement were reduced compared to control embryos (Figure S2G, H). An apparent increase in the occurrence of spiky filopodia-like structures over broader, lamellipodia-like ones (Figure S2I, S2I' and movie S5), reminiscent of what has been described in $\mathrm{ArpC}^{-/-}$murine fibroblast (Suraneni et al., 2015) was also observed.

In contrast to what we observed following CK-666 treatment, in embryos treated from stage 12 with the Formin inhibitor SMIFH2 at $50 \mu \mathrm{M}$, MCCs were properly dispersed and positioned at vertices, but their apical emergence at stage 25 was delayed (Figure 3F, I, J and Figure S2C), in agreement with published data (Sedzinski et al., 2016). Our results thus reveal the importance of actin cytoskeleton dynamics and cell movements in MCC patterning and uncover a possible functional dichotomy between actin branching, required for MCC dispersion, and actin bundling, involved in the apical emergence of MCCs.

\section{The Receptor Tyrosine Kinase Kit is expressed in MCCs, its ligand Scf in outer-layer cells.}

Next, we searched for a molecular mechanism that could sustain self-organised MCC pattern emergence. We reasoned that both homotypic repulsion and affinity towards outer-layer junctions 
must involve cell surface molecules. Our attention was drawn to the Receptor Tyrosine Kinase (RTK) Kit, which has been identified as a transcriptional target of the MCC-inducing factor Multicilin/Mcidas in X. laevis epidermis (Kim et al., 2018; Ma et al., 2014; Stubbs et al., 2012). In addition, its transcripts have been detected by single-cell transcriptomics in X. tropicalis MCCs (Briggs

et

al.,

(https://kleintools.hms.harvard.edu/tools/currentDatasetsList_xenopus_v2.html). As the signalling pathway dependent on Kit and its ligand Stem Cell Factor (Scf, also known as Kitlg, Mast Cell Growth Factor or Steel Factor) is known to control actin cytoskeleton and cell motility, we hypothesized that it might also be involved in controlling the cellular behaviours that underlie the regular pattern of Xenopus MCCs. Whole-mount in situ hybridisation (ISH) with an RNA probe recognizing transcripts of both kit-L and kit-S homeologs detected expression by scattered cells in the non-neural ectoderm starting from early gastrula (stage 10) and persisting through later developmental stages (stage 26) (Figure S3A). FISH on sectioned embryos revealed that in the developing epidermis kit transcripts were exclusively co-expressed with the MCC markers foxj1 and $\alpha$-tubulin (Figure 4A). Conversely, expression throughout the developing non-neural ectoderm has been reported for $s c f-L$ and, at much lower levels, for $s c f-S$ (Goldman et al., 2006; Martin and Harland, 2004) (Figure S3B). RT-PCR on total mRNA from animal caps confirmed the expression of $s c f-L$ from stage 12 to 22 , but failed to detect $s c f-S$ (Figure S3C). FISH revealed that $s c f-L$ transcripts were prevalently localised in the outer layer, with much lower levels of expression in the inner layer (Figure S3B'). In particular, $s c f-L$ expression was rarely detected and only at very low levels in kit-positive MCCs (Figure 4B). Accordingly, single-cell transcriptomics revealed that in X. tropicalis non-neural ectoderm, expression of $s c f$ is highest in goblet cells and excluded from MCCs (Briggs et al., 2018) (https://kleintools.hms.harvard.edu/tools/viewTree.html?tree data/final_180213). In many tetrapods, $\mathrm{Scf}$ is synthesised as a transmembrane molecule but alternative splicing of the exon 6 during mRNA maturation can remove a major serine-protease cleavage site. As a result, 
the exon 6-containing isoform is cleaved to release a soluble peptide, while the exon 6-spliced isoform can only be cleaved at much slower rates at other sites, and thus has a longer half-life as a cell membrane-associated molecule (Brannan et al., 1991; Flanagan et al., 1991; Lennartsson and Ronnstrand, 2012). Both soluble and membrane-anchored isoforms of Scf are able to activate the Kit receptor, albeit with different kinetics and biological outcomes (Gommerman et al., 2000; Lennartsson and Ronnstrand, 2012; Miyazawa et al., 1995; Tabone-Eglinger et al., 2014; TaboneEglinger et al., 2012). RT-PCR with primers encompassing exon 6 showed that only the exon 6containing isoform of $s c f-L$ is detected in developing Xenopus ectoderm (Figure S3C). However, the residues coded by exon 6 in Scf-L and Scf-S have very low similarity to mammalian exon 6 and do not display obvious consensus sites for proteolytic cleavage (Hultman et al., 2007). To address the subcellular localisation of Xenopus Scf, we generated a construct coding for a Cterminal EGFP-tagged version of Scf-L (Figure 4C) and expressed it in Xenopus embryonic ectoderm alone or together with an RFP-tagged version of the tight junction protein ZO-1 (Higashi et al., 2016). Scf-GFP chimeric protein was primarily detected in the lateral plasma membrane of outer ectodermal cells, just basal to ZO1-RFP (Figure 4D, 4D'). However, Western blotting of lysates of Scf-GFP-injected animal caps with an anti-GFP antibody revealed the presence of a peptide compatible with cleavage within exon 6 , together with the uncleaved full-length protein (Figure 4E). We thus assume that both membrane-anchored and soluble forms of Scf-L may be present in the developing Xenopus ectoderm.

\section{Perturbations of the Scf/Kit signal disrupt MCC patterning}

As a first step towards addressing the possible role of Kit signalling in MCC pattern establishment, we took advantage of the Kit tyrosine-kinase pharmacological inhibitor, axitinib (Hu-Lowe et al., 2008). Spinning-disk confocal video microscopy of $\alpha$-tub::LifeActGFP- and mRFP-injected embryos treated with $50 \mu \mathrm{M}$ axitinib from stage 12 showed that MCCs retained their motility (Figure S4A), but displayed a strongly decreased mutual repulsion (Figure 5A and 
movie 8) with a time of contact of $59.20+/-58.15 \mathrm{~min}$ (average+//SD) (Figure 5C). The robust correlation between mutual contact and variation in speed and direction was lost (Figure S4B), and some colliding MCCs continued moving as pairs or groups (Figure S4C-H movie S6 and movie S7). However, neither the cumulative distance covered by MCCs nor their effective displacement were affected (Figure S4I, J), in contrast to what was observed upon CK-666 treatment (Figure S2G, H). In axitinib-treated embryos, MCCs kept crawling along outer-layer junctions (91.07\% of 56 analysed MCCs) (Figure 5C'). An $\alpha$-tub FISH/ZO-1 IF time course on axitinib-treated embryos confirmed that MCCs were largely able to intercalate but did not disperse properly (Figure 5E, 5G-I). As an alternative method to impair Kit activity, we injected a dominant-negative form lacking the TK domain of the receptor (Goldman et al., 2006). Consistently, dn-Kit also caused severe disruption of MCC spacing, although apical emergence and ciliogenesis were largely preserved (Figure S5 and movie S8). Only few Kit-deficient MCCs, often still in direct contact to already inserted ones, were found trapped within the internal layer at stage 25 (Figure S5D').

To further confirm the involvement of Kit in MCC pattern establishment, we designed morpholino-modified antisense oligonucleotides (MOs) to block the translation of kit-L and kit-S mRNAs (MO-ATG-kit-L and MO-ATG-kit-S), or their splicing at the exon2-intron2 junction (MO-splice-kit-L and MO-splice-kit-S) (Figure S6A, S6B). After validation (Figure S6C, S6D), MOs were injected in the two animal ventral blastomeres of 8-cell embryos as cocktails of either MO-ATG-kit-L $+S$ (10ng/blastomere) $\quad$ or $\quad$ MO-splice-kit- $L+S$ (15ng/blastomere). Similar to axitinib-treated and $d n$-kit-injected embryos, $\alpha$-tub FISH and ZO-1 IF at stage 25 showed that kit MO-injected MCCs were properly intercalated but irregularly distributed and present in small clusters (Figure 5F-I). Co-injection of kit MOs with an mRNA coding for full-length Kit-S and carrying seven silent mutations rescued MCC dispersion, thus confirming that this phenotype can be specifically attributed to Kit inactivation (Figure S6E, S6E'). As splicing- and translationblocking kit MOs yielded the same results (Figure S6F, S6F'), all subsequent experiments were 
performed with the latter ones. Thus, spinning-disk confocal video microscopy of embryos injected with $\alpha$-tub::LifeActGFP and 10ng/blastomere kit MOs revealed that MCCs remained motile (Figure S6G), but failed to separate from each other (average time of contact +/- SD: $58.48+/-53.31 \mathrm{~min}$ ) (Figure 5B,C). In addition, they were compromised in their ability to follow outer-layer intercellular junctions (67.14\% of 70 analysed MCCs followed junctions) (Figure 5B,C' and movie 9). Remarkably, kit MO-injected MCCs appeared to emit more filopodia-like than lamellipodia-like structures, similar to CK-666 treatment (Figure 5B' and movie 9).

As Kit function is likely to reflect interaction with its cognate ligand Scf, we then proceeded to address the effect of Scf knockdown on MCC pattern. MOs were designed to block the translation of both $s c f-L$ and $s c f-S$ mRNAs (MO-ATG-scf-L/S) (Goldman et al., 2006), or the exon2-intron2 splicing of the $s c f-L$ mRNA (MO-splice-scf-L) (Figure S7A, S7B). After validation (Figure S7C, S7D), 10ng/blastomere of MO-ATG-scf-L/S was injected in epidermal precursor blastomeres at 8cell stage. $\alpha$-tub FISH and ZO-1 IF at stage 25 showed defects in MCC dispersion (Figure 6A-D), while insertion into the outer layer appeared largely normal (Figure S7E). Injection of 10ng/blastomere of MO-splice-scf- $L$ led to the same phenotype as MO-ATG-scf-L/S (Figure S7F, S7F') and this latter MO was used for all subsequent experiments. Spinning-disk confocal video microscopy of embryos injected with $\alpha$-tub::LifeActGFP and scf MO showed that MCCs remained motile (Figure S7G), but displayed a strongly reduced repulsion (average time of contact +/- SD: 40.31+/-20.04 min) (Figure 6E, 6F and movie 10).

Remarkably, when $s c f \mathrm{MO}$ was injected with mRFP at 16-cell stage to increase mosaicism, MCCs at stage 25 preferentially accumulated at the border of Scf-depleted clones $(78 \%, \mathrm{n}=953)$ (Figure $6 \mathrm{G})$. In contrast $48 \%$ MCCs $(n=1113)$ were found at the borders of control mRFP clones (Figure $\mathrm{S} 7 \mathrm{H})$. Analysis at stage 16 showed that MCCs were initially distributed homogeneously across $s c f$ MO-injected and uninjected areas, suggesting that the observed terminal distribution was due to migration of MCCs outside regions devoid of Scf during the 9 hours separating stages 16 and 25 . Spinning-disk video microscopy of areas straddling clonal boundaries showed that indeed, MCCs 
often left Scf-depleted regions and stopped migrating as soon as they encountered an uninjected outer-layer cell. Interestingly, a majority of MCCs appeared to move across scf MO-injected regions without following outer-layer junctions (only $43.55 \%$ of 62 analysed MCCs followed junctions) (Figure 6H, 6I and movie 11). Strikingly, in silico simulations revealed that MCC accumulation at clonal boundaries could only be recapitulated when both MCC mutual repulsion and affinity towards outer-layer junctions were absent from clones surrounded by normal tissue (Figure 6J and movies 12-14). This suggests that Scf might be able to convey both a repulsive and an adhesive signal.

\section{The Scf/Kit signalling system promotes both adhesive and repulsive functions.}

Our data suggest that the Scf/Kit pathway supports both mutual MCC repulsion and MCC affinity for outer-layer junctions. One tempting mechanistic explanation for this duality is that, as described in other model systems, the soluble and the membrane-anchored forms of Scf each control a distinct function (Gommerman et al., 2000; Jordan and Jackson, 2000; Lennartsson and Ronnstrand, 2012; Miyazawa et al., 1995; Tabone-Eglinger et al., 2014; Tabone-Eglinger et al., 2012). As a first step to test this hypothesis, we ectopically expressed the Scf-GFP chimera in MCCs by means of the $\alpha$-tub promoter. Similar to outer-layer cells (Figure 4D), MCCs displayed a fraction of Scf-GFP signal at the plasma membrane (Figure 7A). We also reasoned that MCCs do not normally express Scf and may lack the enzymes required for its proteolytic processing. $\alpha$ tub FISH at stage 25 showed that large clusters of non-injected MCCs formed around Scf-GFPexpressing MCCs (Figure 7A, B). This suggests that high-level Scf expression in MCCs creates a situation where adhesion dominates repulsion. Spinning-disk video microscopy further confirmed that Scf-GFP-expressing MCCs formed closely packed groups that did not dissociate over periods of more than 3h (Figure 7C and movie 15). Thus, Scf can act as a potent adhesive cue towards Kit-expressing MCCs. 
To address the function of soluble Scf, we generated a construct lacking the MO-binding sequence and carrying a FLAG tag followed by a stop codon immediately 5 ' to the stretch corresponding to exon 6, thus coding a FLAG-tagged version of the extracellular portion of the protein (MOresScfECD-FLAG) (Figure 7D). Western blotting with an anti-FLAG antibody confirmed the presence of the protein in the concentrated supernatant of animal caps injected with MOresScf-ECD-FLAG mRNA, alone or together with MO-ATG-scf-L/S (Figure 7E). While this construct injected alone in 8-cell stage embryos slightly impaired MCC dispersion, it significantly decreased MCC clustering in Scf morphants (Figure 7F-J), suggesting that soluble Scf can by itself promote MCC dispersion.

Finally, we tested whether the presence of the Kit receptor was sufficient to help dispersion of non-MCC intercalating cells. To this aim, embryos were injected with an mRNA coding for fulllength Kit-S and subjected to FISH with a probe against the ionocyte marker, $v 1 a$ (Quigley et al., 2011). In control embryos, ionocytes displayed an irregular pattern of distribution and were often found in adjacent positions (Figure 7K, N). In contrast, kit RNA injection endowed ionocytes with the capacity to disperse and adopt a more regular pattern of distribution (Figure 7L-O). This last set of experiments suggests that the Scf/Kit signalling system is sufficient to promote adhesive and repulsive functions, both necessary to establish a regular pattern of MCC distribution, and that these functions are likely to depend on separable activities of the membrane-anchored and soluble form of Scf, respectively.

\section{DISCUSSION}

\section{Xenopus MCC patterning relies on cell movements}

Although Xenopus MCCs have been the object of extensive investigation in the past years, most studies have focused on their specification within the inner epidermal layer and on the mechanics of their radial intercalation among outer-layer goblet cells. In contrast, much less is known on the mechanisms that control MCC distribution pattern. A pioneering study proposed that the MCC 
scattered pattern results from Notch-mediated lateral inhibition in the inner layer and the constraint of intercalation at outer-layer vertices (Deblandre et al., 1999). However, it was later suggested that the outer layer can accommodate up to twice as many MCCs upon Notch inhibition, with no violation of the non-contiguity rule (Deblandre et al., 1999; Stubbs et al., 2006). This indicates that in normal condition, vertices are in large excess of incoming MCCs and that MCC spacing is not directly linked to the pattern of distribution of vertices. Our data are the first to unambiguously show that MCCs actively move within the inner layer and that motility is a necessary condition of their transition from a random distribution, characterized by extensive inter-MCC contacts, to an orderly pattern, where MCCs are present as isolated cells, each localised in correspondence of an outer-layer vertex. In situations of severe actin or Scf/Kit pathway perturbations, we found that regular spacing between MCCs was lost despite proper intercalation. Thus, we can safely conclude that MCC pattern establishment occurs independently of radial intercalation. In silico simulation defined two conditions required to evenly distribute a population of internal motile cells below epithelial vertices: 1) motile cells must repel each other, thus minimizing their mutual contacts, 2) they must maximize their contacts with outer-layer junctions, so as to stabilize at vertices, which offer the highest local density of junctions. Filming live MCCs in developing embryos allowed us to visualize these two behaviours and to reveal that actin cytoskeleton is involved in both. Immature MCCs in the inner layer extend unpolarized basolateral actin-rich protrusions with which they move and explore the surrounding environment. Whenever two such MCCs come into direct contact, the actin-rich protrusions involved in the contact are retracted, while others are deployed in different regions of the cell body, thus resulting in the two cells moving away from each other. These features are typical of homotypic repulsion mediated by contact inhibition of locomotion, which has previously been shown to promote cellular tiling (Stramer and Mayor, 2016). Concomitantly, immature MCCs also emit apicallypolarized actin-rich protrusions towards junctions between outer-layer cells. Despite the fact that both basolaterally- and apically-directed actin protrusions largely coexist within each MCC, a 
clear temporal shift between the two is observed: over time, the basolateral protrusive activity decreases, while the apically-directed one gains in intensity and stability (see example in Figure $2 \mathrm{C}$ and movie 2). This shift correlates with a progressive decrease of MCC motility within the inner layer (Figure 2C). As a result, by early tailbud stage, all MCCs end up finding a free vertex at regular intervals, to which they anchor and through which they finally intercalate. For a tissuewide regular pattern to emerge from an initial irregular distribution, MCCs are expected to display asynchronous movements and walk for variable distances. These two predictions are supported by our data. First, instant speed measurements indeed reveal that MCCs do move out of phase when their motility is highest (Figure 2B). Second, the displacement of individual MCCs can vary within a 20 -fold range (Figure S4E,I,J).

\section{Self-organised generation of a regular cellular pattern by mutual repulsion and homing.}

Our data show that within the inner layer of the developing Xenopus epidermis, immature MCCs move in a partially random fashion, their free displacements being constrained by mutual repulsion and affinity for outer-layer intercellular junctions, and that these movements are required for the establishment of a regularly-dispersed MCC pattern. Although mathematical simulation allowed us to uncouple mutual repulsion from affinity to junctions, we have so far been unable to do so experimentally. Thus, the contribution of affinity to junctions to the patterning process may appear more difficult to envisage than mutual repulsion. Our interpretation is that affinity to outer-layer junctions is required to balance mutual repulsion, so as to prepare MCCs for intercalation into a partially regular matrix of vertices, constantly subjected to deformations caused by embryo extension, outer cell growth, division or death, events of radial intercalation, and possibly neighbor exchange.

Our work prompts analogies with other instances of developmental patterns emerging from the semi-stochastic movements of cell populations. Phenomena of contact-dependent homotypic repulsion in which mutually contacting cells withdraw projections and/or change direction of 
movement underlie the regular distribution of Cajal-Retzius (CR) neurons (Villar-Cervino et al., 2013) and Retinal Horizontal (RH) cells (Galli-Resta et al., 2002) in mammals, pigment cells in zebrafish (Walderich et al., 2016) and haemocytes in Drosophila (Davis et al., 2012). The case of Xenopus MCC patterning shows nevertheless some specificities. Most notably, MCCs are already present throughout most of the developing epidermis at the start of the patterning process, such that their scattering relies almost exclusively on short-range "wobbling" random movements, without need for a concomitant long-range directional migration as is the case for CR neurons. Consistently, MCCs are unpolarised and lack a well-defined leading process. Moreover, unlike Danio pigment cells, scattering MCCs are post-mitotic and the patterning system does not have to adjust to variations in absolute cell numbers. Finally, in sharp contrast with Drosophila haemocytes, which continue moving and repelling each other at every encounter, even after regular distribution is reached, dispersed MCCs become fixed by anchoring and intercalating into outer-layer vertices. Interestingly, to our knowledge, the only other described case of (semi-) orderly epithelial intercalation of a population of individually dispersed motile cells occurs during the development of the peripheral nervous system in the cephalochordate Amphioxus (BenitoGutierrez et al., 2005; Kaltenbach et al., 2009), but the underlying mechanisms are unknown.

Overall, our work reveals a new instance of self-organised tissue-wide developmental patterning emerging from the interplay of stochastic short-range repulsive and stabilizing intercellular interactions rather than relying on tightly controlled GRNs and classical guidance mechanisms. This is probably the most information-parsimonious strategy to optimise the distribution of a cell population whose function requires that its area of dispersion be covered completely but nonredundantly. Our identification of a molecular pathway responsible for controlling MCC dispersion, independently of cell numbers and differentiation, will make it possible to functionally explore the physiological meaning of such a pattern and the possible outcome of its perturbation.

\section{Implementation of Xenopus MCC patterning by the Scf/Kit pathway.}


The molecular mechanisms controlling homotypic repulsion-mediated patterning have been clearly identified in few cases only. The Eph RTKs and their ephrin ligands control the dispersion of CR neurons in the mammalian cortex (Villar-Cervino et al., 2013). Protocadherins and the immunoglobulin-superfamily proteins Dscams have been involved in the homotypic repulsion phenomena underlying mammalian RH cells tiling (Fuerst et al., 2008; Ing-Esteves et al., 2018), while the scattering of zebrafish pigment cells appears to rely on a variety of cell surface molecules, among which connexins and a potassium channel (Irion et al., 2014; Iwashita et al., 2006).

Our data show that the signal triggered by the interaction between the RTK Kit and its ligand Scf is required to establish the regularly dispersed pattern of Xenopus MCCs. Although this notoriously pleiotropic signalling pathway has been involved in many instances of cell migration, cell movement, cell adhesion, axonal guidance and cytoskeletal rearrangement, this is the first time that it has been shown to play a role in controlling the tissue-wide regular dispersion of a large cell population. Remarkably, kit appears to be a direct transcriptional target of the multiciliogenesis master controller Multicilin/Mcidas (Kim et al., 2018; Ma et al., 2014; Stubbs et al., 2012). Such integration, through a common regulator, of fate determination and motility behaviour may explain how thousands of individual cells can self-organise to achieve a global collective pattern.

Our results are also remarkable in showing that the activity of a single receptor/ligand couple can result in the two opposite but temporally coexisting biological outcomes of MCC mutual repulsion and affinity for outer-layer junctions. Although these two phenomena might seem difficult to reconcile, the Scf/Kit signalling system has already been shown to promote apparently contrasting cell behaviours, such as migration and homing/adhesion, for example in melanoblasts (Jordan and Jackson, 2000; Wehrle-Haller and Weston, 1995), Hematopoietic Stem Cells (HSCs) (Heissig et al., 2002; Tajima et al., 1998) and Primordial Germ Cells (PGCs)(Gu et al., 2009; Gu et al., 2011; Runyan et al., 2006). Currently, the simplest and most widely accepted explanation, supported by 
in vivo, ex vivo and in vitro data, is that the membrane-bound and the soluble forms of the Scf ligand can both bind to and activate the Kit receptor, but with different kinetics, therefore triggering distinct, probably antagonistic signalling pathways and eliciting distinct cellular responses. In relevance to our work, soluble Scf has been shown to lead to transient activation of the Kit intrinsic TK activity, and linked to promotion of cell motility (Jordan and Jackson, 2000; Tabone-Eglinger et al., 2014; Tabone-Eglinger et al., 2012). Conversely, membrane-bound or immobilised Scf leads to formation of long-lived adhesive Scf/Kit complexes at the cell surface, independently of Kit TK activity (Tabone-Eglinger et al., 2014; Tabone-Eglinger et al., 2012). Our data show that the transmembrane Scf can be cleaved in Xenopus epidermis, thus suggesting that the dichotomy between membrane-associated and soluble Scf-dependent signals also controls the balance between adhesion and repulsion required for correct MCC patterning. It is easy to picture that the basolateral localisation of transmembrane Scf in outer-layer cells might favour/stabilise the interaction of Kit-expressing MCCs with intercellular junctions, and even more so with vertices, where the density of the Scf signal is expected to be highest. This view is supported by our observations that MO-mediated depletion of Scf or Kit altered MCC migration along outerlayer junctions. In contrast, axitinib treatment did not cause the same defect, suggesting that TK activity is dispensable for MCC/outer cell interaction, consistent with published evidence (Tabone-Eglinger et al., 2014). Our ectopic expression assay also clearly demonstrated that fulllength Scf can act as a potent adhesive cue towards Kit-expressing MCCs. Incidentally, it also revealed that the patterning system cannot afford Scf to be expressed in MCCs, consistent with our double FISH data, and with transcriptomic analysis suggesting that Mcidas negatively regulates $\operatorname{scf}$ (Ma et al., 2014).

By contrast, it is less simple to envision how soluble Scf can promote MCC mutual repulsion (Figure $7 \mathrm{~J}$ ). One possible explanation is that soluble Scf permeating the inner layer provides a motogenic signal, as described for example for mouse melanoblasts (Jordan and Jackson, 2000), thus keeping MCCs constantly moving around and dispersing until they are anchored to a free 
vertex by the strongly adhesive signal of membrane-bound SCF. However, analysis of the mean instant speed of MCCs in Scf-depleted, Kit-depleted, and axitinib-treated embryos failed to uncover significant differences with control embryos. The total distance covered by MCCs and their overall displacement were not significantly reduced either in axitinib-treated embryos. Altogether, our data suggest that the Scf/Kit pathway controls the actual mutual repulsion phenomenon, rather than the capacity of MCCs to move.

One explanation could be that the interaction between soluble Scf and Kit might initiate a crosstalk with other molecules expressed at the MCC surface and directly responsible for the mutual repulsion response, such as Ephrins and Ephs. However, the remarkable finding that the overexpression of wild-type Kit is sufficient to induce the dispersion of ionocytes suggests that the repulsive activity relies largely on the presence of Kit itself, or that necessary cofactors are not restricted to MCCs. It is thus likely that the signalling cascade connecting Kit to the cytoskeletal effectors of cell repulsion and attraction is a rather direct and short one.

\section{Scf/Kit signalling and MCC actin cytoskeletal dynamics.}

Our data from pharmacological inhibition experiments suggest that different modes of actin polymerization, required for the formation of distinct types of cellular protrusions, control separate aspects of MCC behaviour. Following treatment with CK-666, an inhibitor of the actin-branching factor Arp2/3, MCCs present more filopodia-like extensions, show decreased motility and mutual repulsion and major patterning defects, but no intercalation abnormalities. Interestingly, Arp2/3-/fibroblasts, in which motility only relies on filopodia, also show defects in mutual repulsiondependent cell movements (Suraneni et al., 2012).

On the other hand, treatment with the actin bundling inhibitor SMIFH2 strongly delays intercalation without affecting patterning. The Scf/Kit pathway has been shown to control Arp2/3 activation (Mani et al., 2009) and in our model system its inhibition indeed results in defects partially similar to those due to CK-666 treatment: Kit-deficient MCCs make filopodia-like 
protrusions and present patterning defects but are able to insert into the outer layer. Both CK-666 treatment and Scf/Kit pathway inhibition also increase the duration of MCC mutual contacts. However, unlike CK-666 treatment, Scf/Kit pathway perturbations do not lead to a decrease of MCC motility, again suggesting a direct effect on the mutual repulsion phenomenon. We can thus propose a model in which the main role of the Scf/Kit pathway in Xenopus immature MCCs is the promotion of actin-based lamellipodia formation: Kit activation by soluble Scf would facilitate the formation of transient basolateral lamellipodia, required for redirecting MCC movements away from each other after mutual contact; membrane bound Scf would stabilize the more persistent apical protrusions required for anchoring MCCs to the vertices. Such a model warrants future validation by cell biological and biochemical approaches.

\section{Conclusions}

We show here that, during development, seemingly complex and highly ordered cell patterns can be obtained by the combination of two relatively simple cell behaviours, without need for a highly regulated patterning system. Moreover, deployment of the same signalling system, presumably activated with different kinetics by soluble and membrane-anchored forms of a single ligand, can control both behaviours. This work further stresses the importance of self-organising systems in developmental biology.

\section{ACKNOWLEDGMENTS}

AC was supported by a doctoral fellowship from the French Ministry for Higher Education, Research and Innovation (MESRI). CR was supported by a postdoctoral fellowship from the Turing Centre for Living Systems (CENTURI). The project leading to this publication has received funding from Excellence Initiative of Aix-Marseille University - A*MIDEX, a French “Investments for the Future" program (Projet 'Pépinière d'Excellence 2017 to AP). The work was also supported by funding from Cancéropôle PACA (Programme Emergence 2017 to AP), the 
1 Fondation pour la Recherche Médicale (DEQ20141231765, EQU201903007834 to LK), and the

2 Association pour la Recherche contre le Cancer (PJA 20141201815 to LK). Imaging was

3 performed on PiCSL-FBI core facility supported by the French National Research Agency through

4 the "Investments for the Future" program (France-BioImaging, ANR-10-INBS-04). We are

5 indebted to E. Bazellières, J. Christian, R. Harland, C. Kintner, A. Miller, O. Rosnet and J-P.

6 Tassan for the gift of plasmids. We thank F. Roguet for Xenopus husbandry. We warmly thank

7 Anna Adamiok, Marie Cibois and Marie Zilliox for preliminary experiments, Marc Billaud,

8 Claudio Collinet and Laurence Röder for insights on the project, past and present members of the

9 Kodjabachian laboratory, in particular Camille Boutin and Olivier Rosnet, for discussions, help and technical support, Elsa Bazellières and Vincent Bertrand for insightful comments on the 11 manuscript.

\section{DECLARATION OF INTERESTS}

The authors declare no competing interests.

\section{FIGURE LEGENDS}

Figure 1. The orderly pattern of mature MCCs results from progressive changes in cell distribution. 
A: Fluorescent In Situ Hybridization (FISH) was used to reveal $\alpha$-tubulin-positive immature MCCs (green), combined to immunofluorescence against the tight junction protein ZO-1 to reveal outer-layer intercellular junctions (white). In the course of development, MCCs were increasingly found as isolated, regularly-shaped cells, each localised in correspondence of a single outer-layer vertex. Scale bar is $25 \mu \mathrm{m}$. B: Graph showing the percentage of $\alpha$-tub-positive cells directly in contact with varying numbers of other $\alpha$-tub-positive cells (gray-shaded from 0 to to $\geq 4$ ). $\mathbf{C}$ : Graph showing the number of ZO-1-positive outer-layer vertices superimposable onto the planar projection of each $\alpha$-tub-positive MCC. Whiskers shows the 2.5-97.5 percentiles. D: Graph showing the order index calculated from Delaunay tessellation of the centroids of $\alpha$-tub-positive cells. The order index increased during development, reflecting the progressive transition from a chaotic to an orderly MCC distribution.

Figure 2. MCCs are motile and in silico modelling reveals that mutual repulsion and affinity for outer-layer junctions are required for proper pattern establishment.

A: Spinning-disk confocal video microscopy was used to track $\alpha$-tub::GFP transgenic MCCs within the inner epidermal layer. Still frames from movie 1 are shown. Asterisks point two MCCs that moved under mRFP-injected outer-layer cells. Blue and orange dots serve as landmarks to appreciate MCC mobility. B: Graph showing the instant speed of 55 LifeActGFP-expressing MCCs recorded over a maximum of 8 hours. Dotted light-grey lines: instant speeds of individual MCCs. Black line: mean instant speed of the 55 MCCs; the dark grey area represents the standard error. C: Still frames from movie 2. $\alpha$-tub::LifeActGFP revealed an intense actin-based protrusive activity that progressively shifted from the basolateral to the apical aspect of the cell. For each frame, orthogonal view maximum intensity projection was made on the $\mathrm{x}$ axis (bottom panel) and $\mathrm{y}$ axis (right panel). D-F: Frames from simulation movies 3, 4 and 5, respectively. The black meshwork represents outer-layer intercellular junctions, and red circles the underlying MCCs. The upper row shows the initial random distribution of MCCs, the bottom row shows the final patterns 
obtained under the different regimes. D: In a model where MCC affinity for outer-layer junctions dominated, MCCs localised at vertices, but failed to disperse properly. E: In a model where MCC mutual repulsion dominated, MCCs were regularly distributed, but did not localise at vertices. F: In a model where mutual repulsion and affinity for outer-layer junctions were balanced, MCCs were dispersed and positioned at vertices.

\section{Figure 3. MCCs display both behaviours of mutual repulsion and affinity for outer-layer} junctions.

A: Initial and final frames from movie 6. $\alpha$-tub::LifeActGFP labelling reveals that whenever MCCs come in contact, they moved away from each other. Six MCCs were filmed, of which two $\left(n^{\circ} 1\right.$, red dot and $n^{\circ} 2$, light blue dot) were tracked over the entire duration of the movie. $\mathbf{A}^{\prime}$ : Higher magnification of frames from movie 6 to better show that the collision event between MCCs $n^{\circ} 1$ (red arrowhead) and $\mathrm{n}^{\circ} 2$ (light blue arrowhead) was followed by a change in their direction of movement and by their separation. B: Graph showing the instant speed of MCCs n ${ }^{\circ} 1$ (red line) and $\mathrm{n}^{\circ} 2$ (light blue line) from movie 6. 'C' and ' $\mathrm{S}$ ' indicate contact and separation events, respectively; mathematical exponents label the MCCs from movie 6 to which contact and separation events refer. C: Frames from movie 7 showing an $\alpha$-tub::LifeActGFP-labelled MCC (magenta asterisk) moving along the intercellular junctions among overlying outer-layer cells labelled by mRFP. The blue and the red arrowheads highlight the initial and final position of the labelled MCC. The last panel recapitulates the positions of the observed MCC over $3 \mathrm{~h}$. The black dotted line shows the displacement of the MCC centroid. D-F: Embryos were treated from stage

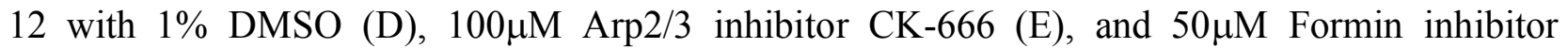
SMIFH2 (F) and analysed at stage 25 by $\alpha$-tub FISH and ZO-1 IF to examine MCC patterning. E: Following CK-666 treatment, MCCs intercalated into the outer layer but were irregularly distributed. F: Treatment with SMIFH2 led to a normal MCC patterning but impaired apical insertion and expansion. In all cases, scale bar is $20 \mu \mathrm{m}$. G: Graph showing the duration of MCC 
mutual contacts in control and CK-666 treated embryos. ${ }^{* * * *}$ p-value $<0.0001$ in a student t-test. H: Graph showing the proportion of MCCs that followed outer-layer junctions over a period of $2 \mathrm{~h}$ in controls, in controls where the outer-layer signal was rotated $90^{\circ}$ counter-clockwise in silico, and in CK-666 treated embryos. I: Graph showing MCC order index in CK-666-treated embryos vs control embryos. J: Graph showing the distribution of the number of contacts between MCCs at stage 25 in control, CK-666- and SMIFH2-treated embryos.

\section{Figure 4. Expression of transcripts encoding Kit and its ligand Scf in the developing Xenopus} epidermis.

A: Double FISH on sectioned embryos with probes against kit (green) and the MCC markers foxJ1 or $\alpha$-tubulin (red) revealed that kit was only expressed in MCCs. B: Double FISH on sectioned embryos showed that $s c f-L$ (red) expression was prominent in outer-layer cells and virtually undetectable in kit-positive MCCs (green). In A and B, DAPI (blue) highlights the nuclei. C: Schematic drawing of the Scf-GFP chimaera, showing the extracellular (EC), transmembrane (TM) and intracellular (IC) domains of Scf-L (light blue), the C-terminal EGFP tag (green), the possible protease cleavage site in exon 6 (red arrowhead), as well as the sizes of the full-length protein and the membrane-retained cleavage product. D: Top view of live outer-layer cells from an embryo injected with mRNAs coding for the Scf-GFP (green) and the RFP-ZO-1 (red) fusion proteins. GFP fluorescence was localised at the membrane. Scale bar is $20 \mu \mathrm{m}$. D': Orthogonal view of the intercellular junction within the dashed white box in D shows that the Scf-GFP chimaera was mainly localised to the basolateral membrane, immediately basal to ZO-1. Scale bar is $5 \mu \mathrm{m}$. E: Lysates from animal caps injected with mRNA coding for either GFP or Scf-GFP were subjected to Western blotting with an anti-GFP antibody. Single and double asterisks point respectively to the $55 \mathrm{kDa}$ full-length $\mathrm{Scf}-\mathrm{GFP}$ and to the $37 \mathrm{kDa}$ band corresponding to the membrane-retained product of proteolytic cleavage at exon 6 . 
Figure 5. Inhibition of Kit activity disrupts MCC patterning.

A: Frames from movie 8. In 50 $\mu \mathrm{M}$ axitinib-treated embryos, LifeActGFP-positive MCCs (green, magenta asterisk) failed to properly separate from each other. B: Frames from movie 9. In MOATG-kit- $L+S$-injected embryos, LifeActGFP-positive MCCs (green, magenta asterisk) failed to properly separate from each other. In A and B, mRFP (red) labelled outer-layer cell membranes. B': A close-up of the boxed area in B, showing highly-branched filopodia-like structures. C: Quantification of MCC mutual contact times in control, axitinib-treated and MO-ATG-kit- $L+S$ injected embryos. $* * * *$ p-value $<0.0001$ in a student t-test. C': Graph showing the proportion of MCCs that followed outer-layer junctions over a period of $2 \mathrm{~h}$ in control, axitinib-treated and kit MO-injected embryos. D-F: $\alpha$-tub FISH (green) and ZO-1 IF (white) showed that compared to control embryos (D) MCCs were irregularly distributed in axitinib-treated (E) and kit MOinjected (F) embryos at stage 25. In F, mRFP mRNA (red) was used as kit MO tracer. In all cases, scale bar is $20 \mu \mathrm{m}$. G: Graph showing the percentage of MCCs intercalated into the outer layer at stages 20, 22 and 25. Intercalation was scored positive when MCCs displayed ZO-1 junctions. Kit inhibition delayed but did not prevent intercalation. H: Graph showing the distribution of the number of contacts between MCCs at stage 25 in control, axitinib-treated and kit MO-injected embryos. The progressive dispersion of $\alpha$-tub-positive MCCs is disrupted in Kit-deficient conditions. I: Graph showing MCC order index at stage 25 in axitinib-treated and kit MO-injected embryos vs control embryos.

\section{Figure 6. Down-regulation of the Scf signal disrupts MCC patterning.}

A, B: $\alpha$-tub FISH (green) and RFP IF (red) revealed that compared to control embryos (A) MCCs were irregularly distributed in scf MO-injected embryo (B) at stage 25. mRFP mRNA was used as scf MO tracer and to outline outer-layer cells. C: Quantification of MCC mutual contacts in control and scf MO-injected embryos. D: Graph showing MCC order index at stage 25 in $s c f$ MOinjected embryos vs control embryos. E: Frames from movie 10. In scf MO-injected embryos, 
LifeActGFP-positive MCCs (green) failed to properly separate from each other. mRFP (red) labelled outer-layer cell membranes. Asterisks label individual MCCs. Dots label two outer-layer cells for reference. F: Graph showing the duration of MCC mutual contact in control and scf MOinjected embryos. ${ }^{* * * *}$ p-value $<0.0001$ in a student t-test. G: $\alpha$-tub FISH (green) and RFP IF (red) of embryos injected with $s c f \mathrm{MO}$ at 16-cell stage and fixed at different developmental times. RFP was used as a MO tracer. MCCs were evenly distributed between MO-positive (red) and MO-negative areas at stage 16 (left column) and accumulated at the boundary between the two areas at later stages (middle and right columns). H: Frames from movie 11, showing a LifeActGFP-positive MCC (yellow asterisk), which left its initial position within the MO-positive area (white arrowhead) and stopped at the boundary with the MO-negative region. In contrast, MCCs outside the MO-positive area did not significantly move. The yellow dotted line shows that the tracked MCC did not follow outer-layer junctions. In all cases, scale bar is $20 \mu \mathrm{m}$. I: Graph showing that a majority of MCCs did not follow outer-layer junctions in scf MO-injected embryos compared to control. J: Frames from simulation movie 12. Mathematical modelling predicted that motile MCCs tend to accumulate at boundaries between regions devoid of both repulsive and attractive signals and in normal regions.

\section{Figure 7. Adhesive and repulsive activities of Scf/Kit signal.}

A: $\alpha$-tub FISH and GFP IF on stage 25 embryos injected with $\alpha$-tub::Scf-GFP shows that noninjected $\alpha$-tub-positive MCCs formed tightly-packed aggregates around Scf-GFP-positive MCCs. Scale bar is $20 \mu \mathrm{m}$. B: Graph showing the distribution of the number of MCC mutual contacts in control and $\alpha$-tub::Scf-GFP injected embryos. C: Frames from movie 15 showing the stability of Scf-GFP-positive MCCs clusters over time. Scale bar is $20 \mu \mathrm{m}$. D: Schematic drawing of the fulllength Scf molecule (top) and of the MOresScf-ECDw/oEx6-FLAG truncated version. E: Western blotting with an anti-FLAG antibody revealed the presence of the FLAG-tagged truncated protein (black arrowhead) in the concentrated supernatant from animal caps injected with the MOresScf- 
ECDw/oEx6-FLAG mRNA alone or together with MO-ATG-scf-L/S. Concentrated supernatant from matched GFP-expressing animal caps was used as a negative control. The non-specific signal at $40 \mathrm{kDa}$ serves as a loading control. F-J: MOresScf-ECDw/oEx6-FLAG restores the dispersion of MCCs in MO-ATG-scf-L/S- injected embryos. F: Control embryos. G: Embryos injected with 30ng MO-ATG-scf-L/S. H: Embryos injected with 10pg MOresScf-ECDw/oEx6-FLAG mRNA. I: Embryos co-injected with 10pg MOresScf-ECDw/oEx6-FLAG mRNA and 30ng MO-ATG-scf$L / S$. In all cases, scale bar is $50 \mu \mathrm{m}$; green: $\alpha$-tub FISH, red: mRFP IF as a tracer. J: Graph showing the number of MCC mutual contacts. ${ }^{* * *}$ p-value $<0.0001$ in a Chi-squared test. K-M: Control embryos (K) and embryos injected with $0.1(\mathbf{L})$ or 1 ng $(\mathbf{M})$ mRNA coding for full length Kit-S, fixed at stage 25 and processed for FISH with the ionocyte marker vla. IF against endogenous ZO-1 $(\mathbf{K})$ or against co-injected mRFP $(\mathbf{M}, \mathbf{N})$ highlighted outer-layer junctions. Kit overexpression led to more even dispersion of ionocytes compared to control. Scale bar is $50 \mu \mathrm{m}$. $\mathbf{N}$ : Graph showing that ionocytes established fewer mutual contacts in Kit-S injected than in control embryos. ${ }^{* * * *}$ p-value $<0.0001$ on a Chi-squared test. O: Ionocyte order index was increased in Kit-S-injected embryos with respect to control embryos.

\section{STAR METHODS}

\section{Ethics statement}

All procedures were performed following the Directive 2010/63/EU of the European parliament and of the council of 22 September 2010 on the protection of animals used for scientific purposes. Experiments on $X$. laevis were approved by the 'Direction Départementale de la Protection des Populations, Pôle Alimentation, Santé Animale, Environnement, des Bouches du Rhône' (agreement number F 13055 21).

\section{Embryo culture and injection}


Ovulation was stimulated in $X$. laevis adult females from NASCO (https://www.enasco.com) by injection of (800 units/animal) Human Chorionic Gonadotropin $\left(\right.$ Chorulon $\left.^{\mathrm{R}}\right)$. On the following day, eggs were recovered by squeezing, fertilized in vitro with sperm from NASCO males, de-jellied in $2 \%$ cysteine hydrochloride $(\mathrm{pH} 8.0)$ and washed, first in water, then in 0.1X MBS (Modified Barth's Saline). Embryos were kept in $0.1 \mathrm{X}$ MBS at $13^{\circ} \mathrm{C}, 18^{\circ} \mathrm{C}$ or $23^{\circ} \mathrm{C}$ until they reached the stage suitable for injection (8-cell or 16-cell), then transferred in 4\% Ficoll in $1 \mathrm{X}$ MBS. Injections were performed using needles made from pulled glass capillaries and mounted on a Nanoject II injector (Drummond). Embryos were injected in animal ventral blastomeres to target the nonneural ectoderm. One blastomere (in 8-cell stage embryos) or two contralateral blastomeres (in 16cell stage embryos) were injected, according to the desired degree of mosaicism. Transgenic $\alpha$ $t u b::$ RFP adult females were purchased from the National Xenopus Resource center.

\section{Plasmids}

\begin{tabular}{|c|c|c|c|c|}
\hline Plamid name & backbone & $\begin{array}{c}\text { linearized } \\
\text { with }\end{array}$ & $\begin{array}{c}\text { amount } \\
\text { injected/blastomere }\end{array}$ & Reference \\
\hline paTub::GFP & pCS2+ & NotI & $50-60 \mathrm{pg}$ & Stubbs et al. 2006 \\
\hline paTub::RFP & pCS2+ & NotI & $50-60 \mathrm{pg}$ & Made in the lab \\
\hline paTub::LifeActGFP & pCS2+ & NotI & $50-60 \mathrm{pg}$ & \\
\hline paTub::LifeActRFP & $\mathrm{pCS} 2+$ & NotI & $50-60 \mathrm{pg}$ & \\
\hline paTub::Scf-GFP & pCS2+ & NotI & $50-60 \mathrm{pg}$ & \\
\hline pCS107-Scf-L-GFP & $\mathrm{pCS} 107$ & NotI & $/ /$ & \\
\hline paTub::MOresKit-S & pCS2+ & NotI & // & \\
\hline pCMV-MOresKit-S & pCMVSport & NotI & $/ /$ & \\
\hline $\begin{array}{l}\text { pMOresScf-ECDw/oEx6- } \\
\text { FLAG }\end{array}$ & $\begin{array}{l}\text { Patented } \\
\text { vector }\end{array}$ & XhoI & // & $\begin{array}{l}\text { Made by e-Zyvec } \\
\text { www.e- } \\
\text { zyvec.com }\end{array}$ \\
\hline
\end{tabular}


2 All $\alpha$-tubulin promoter-based constructs were generated from $\mathrm{p} \alpha$-tub::GFP (a gift from C. Kintner), 3 either with the In-Fusion ${ }^{\mathrm{R}}$ HD Cloning Kit (Takara Bio) or by standard restriction enzyme4 mediated cloning.

5 For In-Fusion cloning, the $\mathrm{p} \alpha-t u b:$ : backbone, excluding the GFP ORF, was PCR amplified using the Phusion ${ }^{\mathrm{R}}$ High-Fidelity DNA Polymerase (New England BioLabs, \#M0530) and the primers patubfor:5'-TCTAGAACTATAGTGAGTCGT-3'

and patubrev:5'CGACCGGTGGATCTGTTGTTGG-3'.

To generate $\mathrm{p} \alpha-t u b:$ RFP, the RFP ORF was PCR amplified from the $\mathrm{pCS} 2+\mathrm{mRFP}$ plasmid (a gift from O. Rosnet) with the Phusion $^{\mathrm{R}}$ High-Fidelity DNA Polymerase and the primers and InFuRFPrev:5'-CACTATAGTTCTAGATTAGGCGCCGGTGGAGTGGCG.

To generate $\mathrm{p} \alpha-t u b::$ LifeActGFP, the LifeActGFP ORF was PCR amplified from the pmEGFPLifeact-7 plasmid (a gift from E. Bazellières) with the Phusion ${ }^{\mathrm{R}}$ High-Fidelity DNA Polymerase and the primers InFuLifeactfor:5'-CAGATCCACCGGTCGTATGGGTGTCGCAGATTTGAT-3' and InFuLifeactGFPrev:5'-CACTATAGTTCTAGATTACTTGTACAGCTCGTCCA-3'.

To generate $\mathrm{p} \alpha-t u b::$ LifeActRFP, the LifeActRFP ORF was PCR amplified from the pmRubyLifeact-7 plasmid (a gift from E. Bazellières) with the Phusion ${ }^{\mathrm{R}}$ High-Fidelity DNA Polymerase and the primers InFuLifeactfor:5'-CAGATCCACCGGTCGTATGGGTGTCGCAGATTTGAT-3' and InFuRFPrev:5'-CACTATAGTTCTAGATTAGGCGCCGGTGGAGTGGCG.

In all the above cases, the vector and insert PCR products were run on agarose/TAE gel, purified with the Macherey-Nagel ${ }^{\mathrm{TM}}$ NucleoSpin $^{\mathrm{TM}}$ Gel and PCR Clean-up kit, then recombined using the In-Fusion ${ }^{\mathrm{R}} \mathrm{HD}$ Cloning Kit according to the manufacturer's instructions.

To make the pCS107-Scf-L-GFP construct, the pCS107-X1Steel1 plasmid (a gift of R. Harland), containing the Scf-L cDNA, was PCR amplified using the Phusion ${ }^{\mathrm{R}}$ High-Fidelity DNA Polymerase and the primers Steellfus-for:5'-GCCACAAATCCCCCTTGTAAAGT-3' and Scf1- 
3'endfus-rev:5'-TATAACACCGACATCAGTTGTGG-3', in order to generate a linearized backbone lacking the scf-L stop codon and 3'UTR. The GFP ORF was PCR amplified from the p $\alpha$ -

$3 t u b:$ GFP construct using the Phusion ${ }^{\mathrm{R}}$ High-Fidelity DNA Polymerase and the primers Scf14 GFPfus-for:5'-GATGTCGGTGTTATAGTGAGCAAGGGCGAGGAGC-3' and GFP3'Scf1 fus5 rev:AGGGGGATTTGTGGCTTACTTGTACAGCTCGTCACT-3'. Vector and insert PCR 6 products were run on agarose/TAE gel, purified with the Macherey-Nagel ${ }^{\mathrm{TM}}$ NucleoSpin ${ }^{\mathrm{TM}}$ Gel and

7 PCR Clean-up kit, then recombined using the In-Fusion ${ }^{\mathrm{R}}$ HD Cloning Kit according to the 8 manufacturer's instructions.

9 The pCS107-Scf-L-GFP plasmid was used both as template for making scf-GFP mRNA and to 10 generate the $\mathrm{p} \alpha-t u b:$ Scf-GFP construct. To this aim, the $s c f-L-G F P$ ORF was PCR amplified from atubSCFGFPfor:5-CAGATCCACCGGTCGTATGAAGAAGACAAAAACTTGG-3' and InFuLifeactGFPrev:5'-CACTATAGTTCTAGATTACTTGTACAGCTCGTCCA-3'. The gelpurified PCR product was recombined into the paTub:: backbone using the In-Fusion ${ }^{\mathrm{R}}$ HD Cloning Kit.

To generate the pCMV-MOresKit-S plasmid, the kit-S ORF was PCR amplified from the Dharmacon/GE clone 4030854 (Xenopus laevis kit-b), using the Phusion ${ }^{\mathrm{R}}$ High-Fidelity DNA Polymerase and the primers atubMOresKitSfor:5-

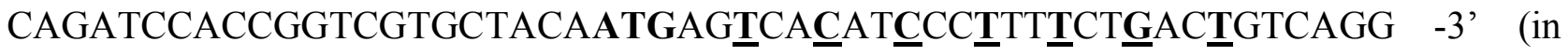
bold, the kit-S start codon, in bold underlined the seven silent mutations introduced to generate mismatches with MO-ATG-Kit-S) and atuBKitSrev:5'CACtAtAGtTCtAGATCAGCCGTCGCTGTTCATCAACAG-3'. The gel-purified PCR product was recombined into the $\mathrm{p} \alpha-t u b:$ : backbone using the In-Fusion ${ }^{\mathrm{R}}$ HD Cloning Kit, thus creating the $\mathrm{p} \alpha-t u b::$ MOresKit-S plasmid. This was subsequently cut with AgeI and NotI and the MOreskit-S ORF-containing fragment was ligated into AgeI/NotI cut pCMVSport6. 
The construct pMOresScf-ECDw/oEx6-FLAG was generated by e-Zyvec (Loos, France; www.ezyvec.com) according to their patented technology. Details on this vector are available upon

3 request.

4 All constructs were made using the StellarTM Competent Cells (Clontech) transformed and grown

5 according to the manufacturer's instructions. Small- and medium-scale plasmid preparations were

6 performed with Macherey-Nagel ${ }^{\mathrm{TM}}$ NucleoSpin $^{\mathrm{TM}}$ Plasmid QuickPure ${ }^{\mathrm{TM}}$ and NucleoBond ${ }^{\mathrm{TM}}$ Extra

$7 \quad$ Midi kits.

\section{mRNA synthesis}

10 Sense mRNAs were synthesized from linearized plasmids with the Ambion mMessage mMachine 11 kit ${ }^{\circledR}$ (Life Technologies) according to the details provided in the table below, then purified with 12 Macherey-Nagel $^{\mathrm{TM}}$ NucleoSpin ${ }^{\mathrm{TM}}$ RNA Clean-up kit. After determination of the concentration, 13 aliquots were kept at $-80^{\circ} \mathrm{C}$.

\begin{tabular}{|c|c|c|c|c|c|}
\hline Plasmid name & Vector & $\begin{array}{c}\text { Linearised } \\
\text { with }\end{array}$ & $\begin{array}{c}\text { Transcribed } \\
\text { with }\end{array}$ & $\begin{array}{c}\text { Amount } \\
\text { injected } \\
\text { /blastomere }\end{array}$ & Reference \\
\hline $\mathrm{pCS} 2+\mathrm{mRFP}$ & pCS2+ & NotI & Sp6 RNA pol & $0,5 \mathrm{ng}$ & \\
\hline pCS2+GFPgpi & pCS2+ & NotI & Sp6 RNA pol & $0,5 \mathrm{ng}$ & $\begin{array}{l}\text { Chartrain et al., } \\
2003\end{array}$ \\
\hline pCS107-Scf-L-GFP & pCS107 & NotI & Sp6 RNA pol & $1 \mathrm{ng}$ & Made in the lab \\
\hline pCS2+RFP-ZO1 & pCS2+ & NotI & Sp6 RNA pol & lng & $\begin{array}{l}\text { Higashi et al., } \\
2016\end{array}$ \\
\hline $\mathrm{pCS} 2+\mathrm{dnKit}$ & pCS2+ & NotI & Sp6 RNA pol & $0,5 \mathrm{ng}$ & $\begin{array}{l}\text { Goldman et al., } \\
2006\end{array}$ \\
\hline pCMV-dnKit-GFP & pCMVSport6 & NotI & Sp6 RNA pol & $0,5 \mathrm{~g}$ & Made in the lab \\
\hline pCMV-MOresKit-S & pCMVSport6 & NotI & Sp6 RNA pol & $0.1-1 \mathrm{ng}$ & Made in the lab \\
\hline
\end{tabular}




\begin{tabular}{|l|l|l|l|l|l|}
\hline pMOresScf- & Patented & Xhol & Sp6 RNA pol & $10 p g$ & Made by e-Zyvec \\
ECDw/oEX6-FLAG & vector & & & & www.e-zyvec.com \\
\hline
\end{tabular}

\begin{tabular}{|c|c|}
\hline MO name & Sequence \\
\hline ATG-kit-L-MO & 5'-TCGTAAAGGATGGAAAGTGGCTCAT-3’ \\
\hline ATG-kit-S-MO & 5'-CCGTAAGGAATGGAATATGGCTCAT-3' \\
\hline kit-S-e2i2spl-MO & 5'-TGTAACCTTATGAATGACTTACCCT-3' \\
\hline kit-L-e2i2spl-MO & 5'-GGAACCCAATGAATGACTTACCCTT-3' \\
\hline ATG-scf-L/S-MO & 5'-GGTAGCTTGTCTATTATCCCCTTAG-3' \\
\hline$s c f-L-e 2 i 2 s p l-\mathrm{MO}$ & 5'-TGGCTAGGTAAAACTCTTACCAGTT-3' \\
\hline
\end{tabular}

\section{Morpholinos}

Morpholinos (MOs) were purchased from GENE TOOLS®. ATG-kit-L-MO and ATG-kit-S-MO were injected together at $10 \mathrm{ng} / \mathrm{MO} /$ blastomere. Kit-S-e2i2spl-MO and Kit-L-e2i2spl-MO were injected together at $15 \mathrm{ng} / \mathrm{MO} /$ blastomere. ATG-Scf-L/S-MO was injected at $10 \mathrm{ng} / \mathrm{blastomere}$ and $S c f-L-e 2 i 2 s p l-\mathrm{MO}$ at $20 \mathrm{ng} /$ blastomere. The blue-tagged version of ATG-Scf-L/S-MO was injected at $20 \mathrm{ng} / \mathrm{blastomere}$.

\section{Pharmacological inhibitors}

Stock solutions of pharmacological inhibitors (all from Sigma-Aldrich ${ }^{\circledR}$ ) were prepared in DMSO and added to embryos cultured in MBS $0,1 \mathrm{X}$ at the stages and concentrations and for the times shown in the following table. Cycloheximide treatment was perform by incubating embryos at stage 14 in MBS $0,1 \mathrm{X}$ containing $300 \mu \mathrm{M}$ cycloheximide two hours before live imaging.

\begin{tabular}{|c|c|c|c|c|}
\hline Drugs & Source & Reference & Concentration & Incubation time and \\
& & & in 0,1X MBS & stage \\
\hline
\end{tabular}




\begin{tabular}{|c|c|c|c|c|}
\hline Axitinib & \multirow{4}{*}{$\begin{array}{l}\text { Sigma- } \\
\text { Aldrich }\end{array}$} & AG-013736 & $50 \mu \mathrm{M}$ & \multirow[t]{3}{*}{ From stage 12} \\
\hline CK-666 & & SML0006 & $100 \mu \mathrm{M}$ & \\
\hline SMIFH2 & & S4826 & $50 \mu \mathrm{M}$ & \\
\hline Cycloheximide & & C7698 & $300 \mu \mathrm{M}$ & From stage 14 \\
\hline
\end{tabular}

1

\section{Apoptosis detection:}

The CellEvent ${ }^{\mathrm{TM}}$ Caspase-3/7 Green Detection Reagent (Invitrogen ${ }^{\mathrm{TM}}$ ) was dissolved in DMSO and added to stage 14 control or cycloheximide-treated embryos in MBS $0,1 \mathrm{x}$ at $20 \mu \mathrm{M}$ final concentration.

\section{In situ hybridization and immunostaining}

Whole-mount chromogenic In Situ Hybridization (ISH) and whole-mount Fluorescent In Situ Hybridization (FISH) were performed as previously detailed (Castillo-Briceno and Kodjabachian, 2014; Marchal et al., 2009). Embryos were fixed in MEMFA (0.1 M MOPS pH 7.4, 2 mM MgSO4, $1 \mathrm{mM}$ EGTA, $3.7 \% \mathrm{v} / \mathrm{v}$ formaldehyde) $1 \mathrm{~h} 30$ at room temperature or overnight at $4{ }^{\circ} \mathrm{C}$ then dehydrated $\mathrm{o} / \mathrm{n}$ in $100 \%$ methanol at $-20^{\circ} \mathrm{C}$. For FISH on section, embryos were fixed in MEMFA, stored in methanol o/n at $-20^{\circ} \mathrm{C}$, rehydrated in PBT (PBS + Tween $\left.0.1 \% \mathrm{v} / \mathrm{v}\right)$, treated with triethanolamine $100 \mathrm{mM}$ and acetic anhydride, incubated in increasing sucrose concentrations, embedded in OCT (VWR Chemicals), cut in $12 \mu \mathrm{m}$-thick cryosections and stored at $-80^{\circ} \mathrm{C}$. Before hybridization, embryos were rehydrated in PBT (PBS $+0.1 \%$ Tween20), treated with Proteinase K ( $8 \mathrm{~min}$ at $2 \mu \mathrm{g} \cdot \mathrm{ml}^{-1}$ ), then, for FISH, the endogenous peroxydase activity was blocked by incubation with $\mathrm{H}_{2} \mathrm{O}_{2} 3 \%$ in PBS for 20 min. For single staining, RNA probes were labelled with digoxigenin-dUTP (Roche). For double staining, various combinations of digoxigenin/fluoresceinlabeled riboprobes were used. For each hybridization, the following amounts of riboprobes were used: $\alpha$-tubulin 1ng; vla $40 \mathrm{ng}$; foxj1 100ng; kit 200ng; scf-L 100ng to 300ng for embryos at stage 
11-16 and stage 19-20, respectively. Hybridization was performed overnight at $60^{\circ} \mathrm{C}$. After hybridization, the embryos were washed at increasing stringency in SSC/0.1\% CHAPS, rinsed extensively in MABX (Maleic Acid Buffered solution $+0.1 \%$ Triton X), then the digoxigeninlabelled probe was revealed through incubation with a sheep anti-DIG antibody conjugated to HRP

5 (POD) (Roche, 1:500). On the third day, embryos were extensively washed in MABX, then staining was revealed using Tyramide Signal Amplification-TSA TM Plus Cyanine 3/5/Fluorescein System (PerkinElmer ${ }^{\circledR}$ ). This reaction was then blocked in a bath of $2 \% \mathrm{H} 2 \mathrm{O} 2$ for $820 \mathrm{~min}$.

9 Following FISH labeling, immunostaining was performed by incubating the embryos in MABX10 BR2 $\%, 15 \%$ FBS with the antibodies listed in the following table. After extensive washes in 11 MABX, embryos were flattened by cutting along the antero-posterior axis and mounted in Mowiol 12 (Sigma-Aldrich) for confocal imaging.

\begin{tabular}{|l|l|l|l|}
\hline \multicolumn{1}{|c|}{ Antibody } & \multicolumn{1}{|c|}{ Species } & \multicolumn{1}{|c|}{ Source } & Dilution \\
\hline ZO1-TJP1 & Mouse IgG1 & Thermo & $1: 200$ \\
& & Scientific & \\
\hline GFP & Chicken & Aves Labs & $1: 500$ \\
\hline RFP & Rat monoclonal & Chromotek & $1: 500$ \\
\hline Alexa Fluor 568 anti-rat & IgG2a & & \\
\cline { 1 - 2 } Alexa Fluor 488 anti-chicken & Goat & Moat & $1: 500$ \\
\cline { 1 - 2 } Alexa Fluor 647 anti-mouse & goat & & $1: 500$ \\
\cline { 1 - 2 } IgG1 & & & $1: 800$ \\
\hline
\end{tabular}


To limit the movement of the embryo during live imaging, control or treated stage 14-15 embryos were embedded in $0,8 \%$ LMP agar in $0,1 \mathrm{X}$ MBS within a small chamber made with two coverslips and silicon grease. Time-lapse imaging was done at $23^{\circ} \mathrm{C}$ starting at stage $14-15$ and for a duration of 2 to 8 hours on a Nikon Roper Spinning-disk Eclipse Ti inverted microscope using a 20X_objective (for apoptosis recording), or 40X/60X_1.25 N.A water-immersion objective (for all the other experiments). Image acquisition was performed with the Meta-Morph software. $20 \mu \mathrm{m}$ deep $Z$ sections were acquired every 0,5 to $2 \mathrm{~min}$ in 0,5 to $0,7 \mu \mathrm{m}$ steps, averaging 2 . To compensate for the fluorescence intensity variations across different injected embryos, the laser power was adjusted for each experiment. Excitation wavelengths were 488nm, $561 \mathrm{~nm}$ and $445 \mathrm{~nm}$ for GFPand RFP- tagged protein and for blue-tagged MO, respectively.

Fixed whole-embryos and sections were examined on Zeiss LSM 510 and 880 confocal microscopes. Four-color confocal Z-series images were acquired using sequential laser excitation, converted into single plane projection and analysed using ImageJ/FIJI software (see image analysis section).

For Scanning Electron Microscopy (SEM), stage 27 control or treated embryos were fixed for $4 \mathrm{~h}$ in $3 \%$ glutaraldehyde in $0.1 \mathrm{M}$ phosphate buffer $\mathrm{pH} 7.4(19 \mathrm{~mL}$ monosodium phosphate $0.2 \mathrm{M}$ and $81 \mathrm{~mL}$ disodium phosphate $0.2 \mathrm{M}$ ), washed in phosphate buffer and filtered bi-distilled water, progressively dehydrated in ethanol at $25 \%, 50 \%$, and $70 \%$ for 30 min each time, then stored in fresh ethanol $70 \%$ at $4{ }^{\circ} \mathrm{C}$ o/n. Embryos were further dehydrated with vigorous agitation once in $90 \%$ ethanol, and twice in $100 \%$ ethanol, for 30 min each, then subjected to $\mathrm{CO}_{2}$ critical point desiccation (CPD030, Balzers) at $40{ }^{\circ} \mathrm{C}$ and $75-80$ bars. Finally, samples were sputter-coated with gold (vacuum $1 \times 10-12$ Torr, beam energy $3-4 \mathrm{keV}$ ) and stuck on the support with conductive glue for immediate SEM digital imaging (FEI TENEO) of the skin epidermis.

\section{Image analysis}

All images were processed with the imageJ/Fiji free software. 
1 Z-stacks from confocal video microscopy were converted into single plane projection by maximum

2 intensity or sum intensity projection. Each channel was processed separately with the optimized z-

3 section corresponding to the outer-/inner-layer cell staining. All visible MCCs were manually

4 tracked with the 'manual tracking' plugin.

5 To overcome the loss of focus, artefactual movements and tissue deformation events, which made

6 the detailed analysis of cell migration and their path tracking impossible, a semi-automatic video

7 refocusing and stabilization procedure was implemented.

8 For each video and for each frame, one user-defined junction was tracked manually using the

9 "Manual Tracking" Fiji plugin, resulting in a set of coordinates of the tracked junction throughout

10 the whole video. This was then passed to a homemade Python script

11 (https://github.com/fabda/mcc paper), which first corrected the coordinates through a 1-

12 dimensional Gaussian filter with sigma=10 to avoid manual tracking artifacts and then quantified

13 the horizontal and vertical drifts between two consecutive frames, using this value to refocus the

14 image by shifting it in the opposite horizontal and vertical direction. A stabilization procedure was

15 then applied to the refocused videos by using the Lucas-Kanade algorithm (Lucas, 1981)

16 implemented into the "Image Stabilizer" $\quad$ Fiji $\quad$ plugin

17 (http://www.cs.cmu.edu/ kangli/code/Image_Stabilizer.html) used with default parameters (see

18 also movie S9).

19 For each refocused and stabilized video, MCC centroids were manually tracked using the "Manual

20 Tracking" Fiji plugin and their position corrected using a one-dimensional Gaussian filter 21 (sigma=10). A set of "behavioral descriptors" were then calculated to help quantify individual cell 22 movements and their interactions throughout a whole movie using a homemade Python script

23 (https://github.com/fabda/mcc paper).

24 For every MCC and at every frame the euclidean distance of the centroids between two 25 consecutive frames was calculated as well as the cumulative euclidean distance from the first 26 frame. The instant speed was defined as the difference of the cumulative sum of the euclidean 
distances between the centroid positions at two consecutive time points divided by the elapsed time.

These values were converted from pixel/frame to $\mu \mathrm{m} / \mathrm{s}$ using the corresponding video acquisition parameter. We also calculated the orientation (in degrees) of the vector made by the MCC centroid and the origin of the coordinate system as the arctangent of the centroid position converted in degrees; the orientation in degree of the vector made by the MCC centroids every five consecutive frames and the origin of the coordinate system as the arctangent of the difference between centroid positions converted into degrees and cardinal directions.

Cell contact events were estimated by counting the time between the first and the last nonambiguous contact time points. Cell contacts already established at the beginning of filming or persisting beyond its end were excluded from quantification.

To characterize the migratory path of MCCs relative to outer-layer cell junctions the following criteria were applied: MCCs emit protrusions in correspondence of outer-layer cells junctions; overlap between MCC shape and outer-layer cells junctions; correlation between direction of MCC migration and orientation of the outer-layer cells junctions. Cells already engaged in radial intercalation with permanent contact with vertices and ambiguous cases were removed from quantification.

Analysis of the apical-basal global cell shape was done through maximum intensity projections of resliced z-stacks following $\mathrm{X}, \mathrm{Z}$ and $\mathrm{Y}, \mathrm{Z}$ orientations with $0,5 \mu \mathrm{m}$ interval from images at the indicated time point. Analysis of Scf-GFP/ZO-1RFP signal localization was done on resliced zstacks from z-series at $0.2 \mu \mathrm{m}$ intervals, taken along chosen intercellular junctions. 3D-projection of intercalating MCCs probing junctions and vertices was made using the 'clear volume' plugin.

To check for the depletion of Scf-GFP protein by a blue-fluorescent tagged version of ATG-scf$L / S$-MO in live we proceeded as follows: mRNAs coding for Scf-GFP and mRFP were co-injected in the two animal ventral blastomeres at stage 8-cell stage, then blue-tagged ATG-scf-L/S-MO was 
injected in two of the four animal ventral blastomeres at 16-cell stage. GFP, RFP and blue fluorescence were recorded at stage 16 .

Images from fixed whole embryos and sections obtained with 510 and 880 LSM confocal microscopes were converted into single plane projection by maximum intensity projections. To calculate MCC and ionocyte mutual contacts and order indexes, as well as the relation of MCCs to vertices and their intercalation, the outlines of individual $\alpha$-tubulin- or vla-positive cells were

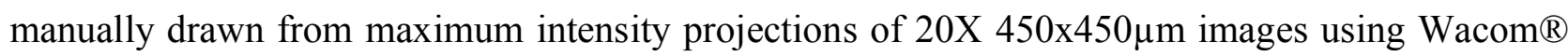
Intuos Pro graphic tablet and FIJI 'ROI manager' tool. Mutual contacts and overlapping to ZO-1positive vertices were manually defined. MCCs were considered intercalated when their apical surface was clearly outlined by a ZO-1 signal. Ambiguous cases were classified as 'non defined' (nd). To calculate the Order Index, cell areas and centroids were defined and the data processed as described in the section 'Mathematical Modelling'.

\section{Western blots}

Animal caps were obtained by manual dissection from stage 10 embryo in 1X MBS and kept in 0,5X MBS until matched control embryos reached stage 17-18, then snap-frozen in liquid nitrogen and stored at $-80^{\circ} \mathrm{C}$ or immediately lysed by boiling for $5 \mathrm{~min}$ in $50 \mathrm{mM}$ Tris- $\mathrm{HCl}(\mathrm{pH} 7.5), 2 \% \mathrm{w} / \mathrm{v}$ SDS, $1 \mathrm{mM}$ DTT. To detect the ScfECDw/oEx6-FLAG protein, 20 injected animal caps were incubated o/n in $400 \mu 10,5 \mathrm{xMBS}$. After recovery, the supernatant was concentrated to $40 \mu \mathrm{l}$ with the Pierce Concentrator PES 10k MWCO system, then stored at $-80^{\circ} \mathrm{C}$. After addition of $5 \mathrm{x}$ Bromophenol blue/Glycerol, samples were loaded on Acrylamide PAGE gels and run using the Hoefer ${ }^{\mathrm{TM}}$ Mighty Small ${ }^{\mathrm{TM}}$ II Mini system with Tris/Glycine/SDS buffer. Transfer to nitrocellulose membranes was performed in Tris/Glycine/Ethanol buffer using the Hoefer ${ }^{\mathrm{TM}}$ TE22 Mini Tank Blotting Unit. Membranes were rinsed with water, stained with Ponceau Red, washed three times for 10min each time in TBS-T (20mM Tris- $\mathrm{HCl}, 150 \mathrm{mM} \mathrm{NaCl}, \mathrm{pH} 7.5,0.05 \% \mathrm{v} / \mathrm{v})$, blocked for $1 \mathrm{~h}$ in TBS-T $+5 \%(w / v)$ non-fat dry milk, then incubated with a rabbit anti-GFP antibody (Torrey 
Pines Biolabs, TP401, 1:1000 in TBS-T + non-fat dry milk) or a rat anti-FLAG antibody (Sigma-Aldrich, clone 6F7, 1:1000 in TBS-T + non-fat dry milk) overnight at $4^{\circ} \mathrm{C}$. After 4 washes $(15 \mathrm{~min}$ each) in TBS-T, membranes were incubated for $1 \mathrm{~h}$ at room temperature with a goat anti-rabbit-HRP-conjugated antibody or a donkey anti-goat-HRP-conjugated antibody (Invitrogen, 1:5000 in TBS-T + non-fat dry milk) then washed four times (15min each) in TBS-T. The signal obtained from enhanced chemiluminescence (Western lightning ECL Pro, Perkin Elmer) was detected with MyECL Imager (Life Technologies).

\section{PCR}

Whole embryos or animal caps were snap frozen at different stages and stored at $-80{ }^{\circ} \mathrm{C}$. Total RNAs were purified with the Qiagen RNeasy kit (Qiagen). RT reactions were carried out using iScript $^{\mathrm{TM}}$ Reverse Transcription Supermix (BIO-RAD). PCRs were carried out with GoTaq ${ }^{\circledR}$ G2 Flexi DNA Polymerase (Promega). Amplification of exon 6 was performed using the primers scfL-ex6for: 5'-GGACCTTGTACCATGCCTGC-3 and scf-L-ex6rev : 5'ATTCCTCTGCCAGGTCTGGA-3 for scf-L; scf-S-ex6for: 5'-TCGGCCTCTTCTTTGTATCG3' and scf-S-ex6rev: 5'-GTACAGTACAGTGTTAATAG-3' for scf-S. To check for the efficiency of kit-S-e2i2spl-MO, the following primers were used: check-splMOKitS1for: 5'ACCATGAACTGGACTTACCTGTGG-3' and check-splMOKitLSrev: 5'CTTCAATGTCCCACTCTGATTTCC-3'. To check for the efficiency of kit-L-e2i2spl-MO the following primers were used: check-splMOKitL1for: 5'-TCCTTTACGATCACAATGAGCTGG3' and check-splMOKitL1rev: 5'-TTACATGAATAGAGAATGTGCTGC-3'.

\section{Statistical analysis}

Graphs were done with Graphpad Prism8 software as well as statistical analysis when indicated using student t-test. Time of contact among MCCs was compared using unpaired parametric 

student t-test and junction following behaviour by Fisher test. The number of samples analysed in 2 all graphs is presented in Table S1. 


\section{$1 \quad$ Mathematical modelling}

2 Geometry

3 The outer layer, considered as a static canvas that can influence the behaviour of two-dimensional $N_{\text {cells }}$

4 MCCs (red disks), is extracted from the segmented image of a ZO-1 IF of a stage-14 control embryo. The

5 number of MCCs injected into simulations was kept constant and was within the range of MCC numbers

6 recorded at stage 14 . The system dynamics was modelled using the effective energies associated with the

7 two mechanisms of MCC mutual repulsion and affinity for outer layer intercellular junctions. The evolution

8 from a random initial state to the final state was guided by an algorithm imposing a progressive decrease of

9 the system total energy. Mathematical expressions for the repulsion and affinity energies were chosen in

10 correspondence with the qualitative behaviours observed in experiments.

12 Energies

13 To account for mutual repulsion, a pair-repulsion energy with an exponential decay on a typical length scale

14 equal to the MCC radius $r_{\text {cell }}$ was chosen. To prevent the overlap among neighbouring MCCs, the repulsion

15 energy was supplemented with a hard-core corresponding to the minimal allowed distance between two

16 cells. Based on experimental observations showing that MCC overlap does not exceed half their size, the

17 hard core was defined as $r_{\text {cell }}$. To ensure that two nearby MCCs undergo a local energy gradient and to

18 prevent aberrant configurations where two MCCs remain stuck with a distance smaller than $r_{c e l l}$, the hard-

19 core energy decreases between $r_{i j}=0$ and $r_{\text {cell }}$.

20 Thus, the pair-repulsion energy reads $E_{r e p}\left(r_{i j}\right)=\left\{\begin{array}{c}k_{r e p} e^{-r_{i j} / r_{c e l l}} \text { if } r_{i j} \geq r_{\text {cell }} \\ A\left(2-r_{i j} / r_{\text {cell }}\right) \text { if } r_{i j}<r_{\text {cell }}\end{array}\right\}$, where $r_{i j}$ is the distance

21 between the centers of MCCs $i$ and $j, r_{c e l l}$ is the MCC radius and $k_{r e p}$ the repulsion constant (positive). The

22 constant $A$ of the hard-core energy is chosen large $(A=100)$, so that the probability for an MCC to be at a

23 distance lower than $r_{\text {cell }}$ from another MCC is very small $\left(e^{-100} \simeq 10^{-44}\right)$. 
1 Affinity for junctions was accounted for with a negative energy term proportional to the overlap between

2 MCCs and outer-layer junctions, calculated as the total pixel intensity of the junction network covered by

3 MCCs. The affinity energy of a cell $i$ is then $E_{a f f}(i)=-k_{a f f} \sum_{(x, y) \in \Omega_{i}} \sigma_{x, y}$ where $k_{a f f}$ is the affinity

4 constant (positive), $\Omega_{i}$ is the set of pixel positions covered by the MCC $i$ and $\sigma_{x, y}$ is the value of the pixel

5 at the position $(x, y)$ of the epithelial canvas. The minus sign ensures that the energy decreases when the

6 total pixel intensity covered by the MCC $i$ increases.

7 To build the epithelial canvas used to calculate the affinity energy, a Gaussian blur of a size equal to a MCC

8 diameter was applied to the segmented epithelium matrix. This considerably fastens the simulations by

9 directing MCCs towards junctions thanks to an energy gradient, without changing the final state. Note that

10 this could also reflect that regions lining junctions are mechanically more favourable, as the height of the

11 epithelial cells is minimal at junctions.

\section{Dynamics}

14 The total energy of the system is the sum of the repulsion and affinity energies of all the MCCs $E_{\text {tot }}=$ $15 \sum_{i} E_{a f f}(i)+\sum_{i \neq j} E_{r e p}\left(r_{i j}\right)$ where $i$ and $j$ refer to MCCs. Monte-Carlo simulations of the system were 16 performed with a Metropolis algorithm to impose the energy minimization constraint. At each time step of 17 the simulation a random displacement was computed and attributed to each MCC. This displacement 18 corresponds to a diffusive motion with a diffusion constant yielding typical jumps of one-tenth of a cell19 radius distance. Moves were accepted or rejected following a Metropolis algorithm depending on the value 20 of $\Delta E_{\text {tot }}=E_{\text {tot }}($ after $)-E_{\text {tot }}$ (before), the difference between the system total energy after and before 21 each move. If the total energy has decreased $\left(\Delta E_{t o t}<0\right)$ the move is accepted. If the total energy has 22 increased $\left(\Delta E_{\text {tot }}>0\right)$ the move is rejected in most cases but has a nonzero probability to be accepted if the 23 increase is of the order of the thermal energy $k_{B} T\left(k_{B}\right.$ is the Boltzmann constant and $T$ the temperature, 24 both set to 1 in our simulations), with a probability given by $\exp \left(-\Delta E_{\text {tot }} /\left(k_{B} T\right)\right)$. The virtual-time of the 
1 simulation is increased by one after $N_{\text {cells }}$ such dynamical steps (one Monte-Carlo time step). The

2 simulation was run for a Monte-Carlo time sufficient for the system to reach a stationary final state.

3 Depending on the respective weight of the energy constants $k_{r e p}$ and $k_{a f f}$ final states with different

4 qualitative patterning of the MCCs were generated. Let alpha be the ratio between energy constants, $\alpha=$

$5 k_{\text {rep }} / k_{\text {aff }}$. When $\alpha \gtrsim 10^{7}$, the total energy of the system $E_{\text {tot }}$ is dominated by the repulsion energy. When

$6 \alpha \lesssim 10^{3}$, the total energy of the system is dominated by the affinity energy.

8 Quantification

9 In order to have a more quantitative description of the system state, two indices were defined, to quantify

10 the MCC spatial order and their localization at junctions, respectively.

11 The order index $I_{o}$ was computed from Delaunay tessellation of MCC positions. The position of each MCC

12 is computed as a single point, located at its centroid. Systems with different MCC densities $\rho_{\text {cells }}$ (defined

13 as the ratio between the total area occupied by MCCs and the available area of the epithelium) were

14 distinguished, since the number of possible final MCC organisations increases with the available space.

15 The order index $I_{o}$ is a measure of the narrowness of the distribution of areas $A_{D e l}$ of Delaunay triangles

16 while accounting for the available space via $\rho_{\text {cells }}: I_{o}=\left(1-\rho_{\text {cells }}\right)\left(1-\frac{\operatorname{sd}\left(A_{D e l}\right)}{\operatorname{mean}\left(A_{D e l}\right)}\right)$.

$17 I_{o}$ is minimal when the standard deviation of Delaunay areas is of the order of its mean and is large when

18 the standard deviation is small compared to the mean. Therefore $I_{o}$ is maximal when MCCs are perfectly

19 ordered and minimal when they are most disordered. Note that this definition of order was used both for

20 simulations and experimental data.

21 The covering index $I_{c}$, representing the extent of overlapping between outer-layer junctions and MCCs, was

22 computed as the total pixel intensity of the epithelium covered by MCCs, $\sum_{i} \sum_{(x, y) \in \Omega_{i}} \sigma_{x, y}$, minus a noise

23 term. This noise term, corresponding to the background value of covered pixels in a purely random

24 configuration of MCCs, was computed by averaging the pixels $\sigma_{x, y}$ of the whole epithelium and multiplying

25 this value by the cell surface $i_{c}^{\text {noise }}=\operatorname{mean}\left(\sigma_{x, y}\right) \pi r_{c e l l}^{2}$. The covering index was normalized by a term 
1 accounting for the maximal possible extent of overlapping of junctions by one MCC. This was obtained by

2 finding the maximal possible overlapping value for a cell $C$ scanning among all possible positions $\left(x_{C}, y_{C}\right)$

3 of its center $i_{c}^{\max }=\max _{\left(x_{C}, y_{C}\right)} \sum_{(x, y) \in \Omega_{C}} \sigma_{x, y}$.

4 Thus the covering index is $I_{c}=\frac{N_{c e l l s}^{-1} \sum_{i} \sum_{(x, y) \in \Omega_{i}} \sigma_{x, y}-i_{c}^{\text {noise }}}{i_{c}^{\text {max }}-i_{c}^{\text {noise }}}$.

5 The index is thus minimal when the overlap of MCCs with junctions does not exceed the overlap obtained

6 from a random scattering of MCCs. It is maximal when the overlap is the highest possible.

7
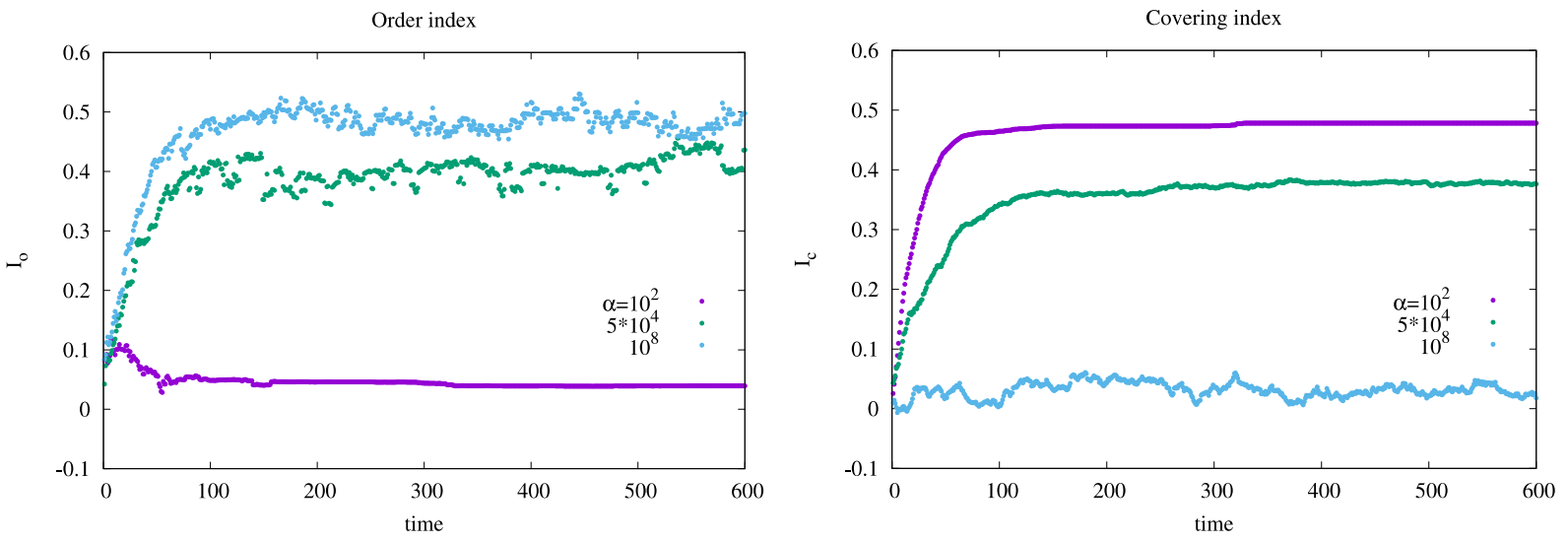

8

9 Order and covering index evolution with time for different values of $\alpha=k_{r e p} / k_{a f f}$.

10 In the repulsion dominated regime $\left(\alpha=10^{8}\right)$ MCCs evolve toward a strict ordering regardless of

11 junctions hence the order index is large while the affinity index is small. In the affinity-dominated

12 regime $\left(\alpha=10^{2}\right)$, MCCs finally localize under junctions/vertices regardless of other MCCs, yielding

13 a large affinity index while the order index remains small. In the intermediate regime $\left(\alpha=5 \times 10^{4}\right)$

14 the two indices take intermediate values. 
Order and covering indexes

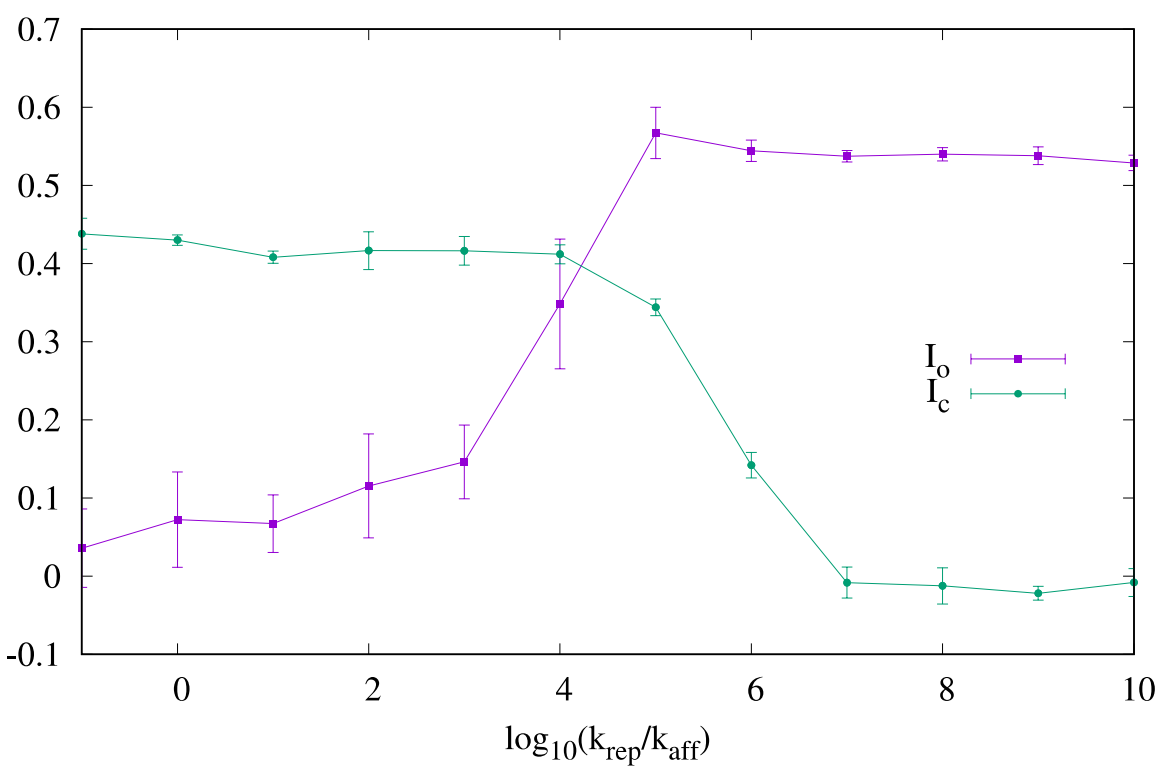

1

2 Final values of order and affinity indices for different simulation conditions.

3 The points represent mean values computed over five simulations and the error-bars represent the

4 standard deviation.

5

6 Clonal simulations

7 In clonal simulations, where patches of the epithelium lack either or both repulsion and affinity, the set of

8 clone regions was called $Z$, and the new interaction constants $k_{r e p}^{Z}$ and $k_{a f f}^{Z}$. The energies depending on the

9 position of the MCCs (inside or outside $Z$ ) were thus modified.

10 The repulsion energy reads

11

$$
E_{r e p}\left(r_{i j}\right)=\left\{\begin{array}{c}
k_{\text {rep }} \exp \left(-r_{i j} / r_{\text {cell }}\right) \text { if }(i, j) \notin Z \\
k_{\text {rep }}^{Z} \exp \left(-r_{i j} / r_{\text {cell }}\right) \text { if }(i, j) \in Z \\
\sqrt{k_{r e p} k_{r e p}^{Z}} \exp \left(-r_{i j} / r_{\text {cell }}\right) \text { if }(i \in Z \text { and } j \notin Z) \text { or }(j \in Z \text { and } i \notin Z)
\end{array}\right\}
$$

12 for $r_{i j} \geqslant r_{\text {cell }}$ (the hard-core energy of $E_{r e p}\left(r_{i j}\right)$ in section Energies is unchanged when $r_{i j}<r_{c e l l}$ ), and the

13 affinity energy reads $E_{a f f}(i)=-\sum_{(x, y) \in \Omega_{i}} k_{a f f}(x, y) \sigma_{x, y}$ with $k_{a f f}(x, y)=\left\{\begin{array}{l}k_{a f f} \text { outside } Z \\ k_{a f f}^{Z} \text { inside } Z\end{array}\right\}$ 
2 Movie 1: MCCs move actively within the plane of the ectoderm inner layer.

3 Movie 2: Dynamic behaviour of the actin cytoskeleton in immature MCCs.

4 Movie 3: In a simulation dominated by MCC affinity for outer-layer junctions, MCCs home to 5 vertices, but do not disperse properly.

6 Movie 4: In a simulation dominated by MCC mutual repulsion, MCCs disperse properly, but do not 7 home to vertices.

8 Movie 5: In a simulation where MCC mutual repulsion and affinity for outer-layer junctions are 9 balanced, MCCs disperse and home correctly to vertices.

10 Movie 6: When MCCs come into contact via lamellipodia-like structures, they withdraw from each other. $\alpha$-tub::GFP transgenic MCCs within the inner epidermal layer are tracked relative to mRFPinjected outer-layer cells. Red and dark blue dots and lines show the position and migration path of cell $n^{\circ} 1$ and $n^{\circ} 2$, respectively (see Figure $3 A$ ). Arrows point contact between cell $n^{\circ} 1$ and $n^{\circ} 2$ (white) or cell $n^{\circ} 1$ and $n^{\circ} 3$ (red), which are followed by changes in the direction of cell migration.

Movie 7: MCCs preferentially move along outer-layer intercellular junctions.

Movie 8: MCC mutual repulsion is strongly decreased in axitinib-treated embryos.

Movie 9: MCC mutual repulsion is strongly decreased in kit MO-injected embryos.

Movie 10: MCC mutual repulsion is strongly decreased in scf MO-injected embryos.

Movie 11: In $s c f$ mosaic morphants, MCCs leave Scf-depleted regions without following outerlayer intercellular junctions. The centroid of the tracked MCC is marked 1, the initial position within the clone is marked 2. The mark 3 points an MCC, which remains associated to the border but never enters the Scf-depleted region.

Movie 12: Mathematical modelling predicts that MCCs tend to accumulate at boundaries between regions devoid of both repulsion and affinity, and in normal regions.

Movie 13: When only repulsion is suppressed in clones surrounded by normal regions, MCCs remain within the clones but do not disperse homogeneously. 
$1 \quad$ Movie 14: When only affinity is suppressed in clones surrounded by normal regions, MCCs disperse

2 homogeneously within the clones but do not preferentially associate to vertices.

3 Movie 15: MCCs overexpressing Scf-GFP form stable aggregates.

$4 \quad$ Movie S1: Fluorescent labelling of activated caspases 3 and 7 reveals no apoptotic cell death within 5 the ectoderm.

6 Movie S2: When MCCs come into contact via large cell protrusions, they withdraw from each other.

7 Movie S3: Tilted 3D projection from movie 7 showing MCC apically-directed protrusions 8 occurring in correspondence of outer-layer intercellular junctions.

9 Movie S4: MCC mutual repulsion is strongly decreased in CK-666-treated embryos.

10 Movie S5: CK-666-treated embryos strongly decreased MCC mutual repulsion and increase the 11 occurrence of spiky filopodia-like structures over broader, lamellipodia-like ones.

12 Movie S6: The colliding MCCs of control embryos separate through mutual repulsion. A large cohort of LifeActGFP-positive MCCs with low mosaicism (left panel) was tracked for two hours (right panel) showing that colliding MCCs move away from each other and change their direction of migration. This behaviour was visible for 30 out of 36 contacts among 18 cells over a period of

162 hours.

17 Movie S7: MCCs move as pairs or groups in axitinib-treated embryos. A large cohort of 18 LifeActGFP-positive MCCs with low mosaicism (left panel) was tracked for two hours (right panel), showing that Kit-deficient MCCs failed to separate and move as pairs or groups. Only 10 events of repulsion were visible out of 22 observed contacts among 18 cells over a period of 2 21 hours.

Movie S8: MCC mutual repulsion was strongly decreased in dn-Kit-injected embryos.

Movie S9: Example of a movie subjected to our refocusing and stabilisation procedures (see STAR Methods). 
Figure S1. Cell death and cell fate reversal are unlikely to play a major role in establishing the MCC regular pattern.

A: Stage 15 mRFP-injected embryos were incubated with the CellEvent ${ }^{\mathrm{TM}}$ Caspase-3/7 Green Detection Reagent to detect activation of the executioner caspases 3 and 7 in presence of $300 \mu \mathrm{M}$ cycloheximide as an apoptosis inducer (Trindade et al., Development, 2003) or in DMSO as a control and filmed for 3 hrs (right). Apoptotic cells (green) are found in the epidermis of cycloheximide-treated embryos (left), but not in control ones, even after prolonged observation (right). Embryos were injected with mRFP (red) to allow a better visualisation of cell outlines. Scale bar is $20 \mu \mathrm{m}$. B: $\alpha$-tub::RFP transgenic embryos were fixed at different developmental stages and subjected to IF with an anti-RFP antibody (red, top row) and FISH against $\alpha$-tubulin (green, middle row). Merging the two channels (bottom row) shows few $\alpha$-tub-positive/RFPnegative cells (white arrowheads), but fails to uncover any RFP-positive/ $\alpha$-tub-negative cells. DAPI staining (blue, bottom row) allows the identification of nuclei. Scale bar is $20 \mu \mathrm{m}$. C: Venn diagrams showing the overlap of the GFP- and RFP-positive cell populations at different developmental stages.

\section{Figure S2. Role of actin cytoskeleton in MCC patterning.}

A: Frames from movie S2 show LifeActGFP-expressing MCCs establishing a transient contact via lamellipodia-like structures (red arrowheads) that are subsequently retracted. The yellow arrowhead points to an example of thinner, filopodia-like structures. Scale bar is $20 \mu \mathrm{m}$. B: A tilted 3D projection from movie S3 showing that apically directed protrusion (white arrowheads) correspond to outer-layer cell junctions. C: Quantification of MCC intercalation at stage 19 and stage 25 in control embryos and embryos treated with CK-666 or SMIFH2. D: Graph showing the instant speed of 40 LifeActGFP-expressing MCCs from CK-666 treated embryos, recorded over a maximum of 4 hours. Dotted light-grey lines: instant speeds of individual MCCs. Black line: mean instant speed of the $40 \mathrm{MCCs}$; the dark grey area represents the standard error. E: Initial and 
final frame from movie S4 showing three LifeActGFP-expressing MCCs, which fail to separate over the course of two hours in a CK-666-treated embryo. Scale bar is $20 \mu \mathrm{m}$. F: Graph showing the instant speed of two MCCs (1 in light blue, and 2 in red) from movie S4. G: Graph plotting the cumulative path distances covered by MCCs from control and CK-666-treated embryos. H: Graph plotting the displacements (based on initial and final positions) of MCCs from control and CK666-treated embryos. I: Frames from movie S5 show that MCCs in CK-666 treated embryos show decreased mutual repulsion; I': A close-up on the outlined area from I, to highlight filopodia-like structures (white arrowheads).

\section{Figure S3. Expression of kit and scf in Xenopus epidermis.}

A: Whole-mount ISH with a probe against kit- $L$ and kit-S shows expression in scattered nonneural ectoderm cells at stages 10 to 26 . Anterior is to the top for stages 10 and 14 , to the left for stages 18 and 26. B: Whole-mount ISH with a probe against $s c f-L$ reveals expression throughout the ectoderm from stages 14 to 22 . Anterior to the left. B': FISH on a sectioned embryo at stage 25 shows stronger $s c f-L$ expression (red) in the ectoderm outer layer. DAPI (blue) stains the nuclei. C: RT-PCR on total RNA from animal caps at different developmental stages with primers encompassing the exon6 region of $s c f-L$ (left) or $s c f-S$ (right) amplifies a $141 \mathrm{bp}$ band, only for $s c f-$ $L$, which is present at all developmental stages analysed. Plasmids containing the full-length cDNA of either $s c f-L$ (pCMV-Sport6-SCF-L) or $s c f-S$ (pCMV-Sport6-SCF-S) were used as positive controls for amplification. Molecular weight marker is GeneRuler 100bp Plus DNA Ladder.

\section{Figure S4. Inhibition of Kit signalling by axitinib treatment does not affect MCC motility. A:} Graph showing the instant speed of 67 LifeActGFP-expressing MCCs recorded over a maximum of 4 hours in axitinib-treated embryos. Dotted light-grey lines: instant speeds of individual MCCs. Black line: mean instant speed of the 67 MCCs; the dark grey area represents 
the standard error. B: Graph showing the instant speed of two MCCs from movie S7. C: Frames from movie S6 showing LifeActGFP-expressing MCCs from a control embryo. D: Tracking of MCCs from movie S6, showing their paths over a duration of 2 hours. E: Graph showing the initial and the final positions of the tracked MCCs from movie S6. F: Frames from movie S7 showing LifeActGFP-expressing MCCs from an axitinib-treated embryo. G: Tracking of MCCs from movie S7, showing their paths over a duration of 2 hours. H: Graph showing the initial and the final positions of the tracked MCCs from movie S7. The thick coloured arrows correspond to MCCs that did not separate during their displacement. I: Graph plotting the cumulative path distances covered by MCCs from control and axitinib-treated embryos. J: Graph plotting the displacements (based on initial and final positions) of MCCs from control and axitinib-treated embryos.

\section{Figure S5. Expression of dominant-negative Kit affects MCC distribution.}

A, B: Whole-mount ISH with a probe against $\alpha$-tubulin shows a normal MCC distribution in stage 27 control embryos (A) and patterning irregularities in age-matched $d n$-kit-injected embryos (B). A' and B' are close-ups of A and B respectively. C, D: Whole-mount FISH with a probe against $\alpha$-tubulin (white) in control (C) and $d n$-kit-injected (D) stage 27 embryos. mRNA coding for GFP (green) is used as a tracer for $d n$-kit mRNA, DAPI (blue) stains nuclei. Scale bar is $200 \mu \mathrm{m}$. C' and D' are close-ups of $\mathbf{C}$ and $\mathbf{D}$, respectively, showing the irregular clustering of MCCs in $d n-k i t-$ injected embryos. Scale bar is $20 \mu \mathrm{m}$. E, F: Scanning electron microscopy images of control (E) and $d n$-kit-injected (F) stage 27 embryos. E' and $\mathbf{D}^{\prime}$ are close-ups of $\mathbf{E}$ and $\mathbf{D}$, respectively, showing that MCCs in $d n$-kit-injected embryos are irregularly clustered but do produce cilia. G: Frames from movie S8, showing that LifeActGFP-positive MCCs fail to separate properly in $d n$ kit-injected embryos. mRNA coding for RFP (red) serve as tracer for $d n$-kit mRNA. Scale bar is $20 \mu \mathrm{m}$. 
A: Morpholino Oligos against kit-L. 1) Large font size underlined: kit- $L$ exon 1; small font size: 5'UTR; small font size italic: intron 1; in red the stretch targeted by MO-ATG-kit-L. 2) Large font size underlined: kit-L exon 2; small font size italic: intron 2 ; in red the stretch targeted by MOsplice-kit-L. B: Morpholino Oligos against kit-S. 1) Large font size underlined: kit-S exon 1; small font size: 5'UTR; small font size italic: intron 1; in red the stretch targeted by MO-ATG-kit-S. The asterisk/arrowheads point to the seven bases mutated to generate MOres-kit-S. 2) Large font size underlined: kit-S exon 2; small font size italic: intron 2; in red the stretch targeted by MO-splice-

kit-S. C: Lysates from animal caps injected with mRNA coding for GFP and mRNA coding for a C-terminally GFP-tagged dominant-negative form of kit-S alone or together with MO-ATG-kit$L+S$ were subjected to Western blotting with an anti-GFP antibody. The red arrowhead points to a GFP-positive band corresponding to the intracellular fragment generated by proteolytic cleavage of the dn-Kit-GFP protein, which is lost in embryos co-injected with MO-ATG-kit-L+S. D: RTPCR on kit-L and kit-S to test the action of the splice-blocking MOs; a.c.: animal caps; w.e.: whole embryos; $0.8 \%$ agarose/TAE gel. The injection of MO-splice-kit-S or MO-splice-kit- $L$ results in a decrease of the levels of kit-S and kit-L mRNAs, respectively. E: $\alpha$-tub FISH (green) and RFP IF (red) on stage 25 embryos show, from left to right, normal MCC distribution in control embryos and in embryos injected with mRNA coding for morpholino-resistant form MOres-Kit-S alone, abnormal MCC clustering in embryos injected with MO-ATG-kit- $L+S$, and recovery of normal MCC distribution in embryos co-injected with MO-ATG-kit- $L+S$ and MOres-kit-S mRNA. In all cases, mRNA for RFP was used as an injection tracer. Scale bar is $20 \mu \mathrm{m} . \mathrm{F}: \alpha$-tub FISH (green) and ZO-1 IF (white) on control embryos (left panel) and embryos injected with either MO-ATGkit $L+S$ (middle panel) or MO-splice-kit- $L+S$ (right panel) shows that both ATG- and splicetargeting MOs lead to the same phenotype of irregularly distributed and clustered MCCs at stage 25. mRFP mRNA (red) was used as MO tracer. Scale bar is $20 \mu \mathrm{m}$. The graph in F' shows a quantification of the abnormal MCC clustering in MO-ATG-kit- $L+S$ and MO-splice-kit- $L+S$ 
injected embryos. G: Graph showing the instant speed of 40 LifeActGFP-expressing MCCs in MO-ATG-kit- $L+S$-injected embryos, recorded over a maximum of 8 hours. Dotted light-grey lines: instant speeds of individual MCCs. Black line: mean instant speed of the 40 MCCs; the dark grey area represents the standard error.

\section{Figure S7. Characterisation of Morpholino Oligos against $s c f-L$ and $s c f-S$.}

A: Morpholino Oligos against $s c f-L$. 1) Large font size underlined: $s c f-L$ exon 1; small font size: 5'UTR; small font size italic: intron 1; in red the stretch targeted by MO-ATG-scf-L/S. 2) Large font size underlined: $s c f-L$ exon 2; small font size italic: intron 2 ; in red the stretch targeted by MO-splice-scf-L. B: Morpholino Oligos against $s c f-S$. 1) Large font size underlined: $s c f-S$ exon 1; small font size: 5'UTR; in red the stretch targeted by MO-ATG-scf-L/S. C: En face view of live outer-layer cells from a stage 18 embryo co-injected with mRNAs coding for Scf-GFP (green) and RFP (red), as well as a blue-tagged version of MO-ATG-scf-L/S. The Scf-GFP signal is lost from the MO-receiving cells, while the RFP signal is unchanged. Scale bar is $20 \mu \mathrm{m}$. D: Lysates from animal caps injected with mRNA coding for GFP, Scf-GFP and Scf-GFP together with MO-ATG$s c f-L / S$ were subjected to Western blotting with an anti-GFP antibody. Bands corresponding to the $55 \mathrm{kDa}$ full-length Scf-GFP and to the $37 \mathrm{kDa}$ exon 6 proteolytic cleavage product (arrowheads) are absent from MO-ATG-scf-L/S injected embryos. E: Quantification of the MCC intercalation in control and MO-ATG-scf-L/S-injected embryos. F: $\alpha$-tub FISH (green) and RFP (red) IF on control embryos (left panel) and embryos injected with either MO-ATG-scf-L/S (middle panel) or MO-splice-scf-L (right panel) shows that both ATG- and splice-targeting MOs lead to the same phenotype of irregularly distributed and clustered MCCs at stage 25. mRFP mRNA (red) was used as MO tracer. Scale bar is $20 \mu \mathrm{m}$. F' shows a quantification of the abnormal MCC clustering in MO-splice-scf-L/S- and MO-ATG-scf-L/S-injected embryos. G: Graph showing the instant speed of 61 LifeActGFP-expressing MCCs in MO-ATG-scf-L/S-injected embryos, recorded over a maximum of 4 hours. Dotted light-grey lines: instant speeds of individual MCCs. Black line: 


\section{References}

Belin, B.J., Goins, L.M., and Mullins, R.D. (2014). Comparative analysis of tools for live cell imaging of actin network architecture. Bioarchitecture 4, 189-202.

Benito-Gutierrez, E., Nake, C., Llovera, M., Comella, J.X., and GarciaFernandez, J. (2005). The single Amphitrk receptor highlights increased complexity of neurotrophin signalling in vertebrates and suggests an early role in developing sensory neuroepidermal cells. Development 132, 2191-2202.

Brannan, C.I., Lyman, S.D., Williams, D.E., Eisenman, J., Anderson, D.M., Cosman, D., Bedell, M.A., Jenkins, N.A., and Copeland, N.G. (1991). SteelDickie mutation encodes a c-kit ligand lacking transmembrane and cytoplasmic domains. Proc Natl Acad Sci U S A 88, 4671-4674.

Briggs, J.A., Weinreb, C., Wagner, D.E., Megason, S., Peshkin, L., Kirschner, M.W., and Klein, A.M. (2018). The dynamics of gene expression in vertebrate embryogenesis at single-cell resolution. Science 360.

Briscoe, J., and Small, S. (2015). Morphogen rules: design principles of gradient-mediated embryo patterning. Development 142, 3996-4009.

Castillo-Briceno, P., and Kodjabachian, L. (2014). Xenopus embryonic epidermis as a mucociliary cellular ecosystem to assess the effect of sex hormones in a non-reproductive context. Front Zool 11, 9.

Chung, M.I., Kwon, T., Tu, F., Brooks, E.R., Gupta, R., Meyer, M., Baker, J.C., Marcotte, E.M., and Wallingford, J.B. (2014). Coordinated genomic control of ciliogenesis and cell movement by RFX2. Elife 3, e01439.

Cibois, M., Luxardi, G., Chevalier, B., Thome, V., Mercey, O., Zaragosi, L.E., Barbry, P., Pasini, A., Marcet, B., and Kodjabachian, L. (2015). BMP signalling controls the construction of vertebrate mucociliary epithelia. Development 142, $2352-2363$.

Cibois, M., Scerbo, P., Thomé, V., Pasini, A. and Kodjabachian, L. (2014). Induction and Differentiation of the Xenopus Ciliated Embryonic Epidermis. In Xenopus Development, M.K.a.J.Z. Kubiak, ed. (Oxford: John Wiley \& Sons, Inc) .

Davidson, E.H. (2010). Emerging properties of animal gene regulatory networks. Nature 468, 911-920.

Davis, J.R., Huang, C.Y., Zanet, J., Harrison, S., Rosten, E., Cox, S., Soong, D.Y., Dunn, G.A., and Stramer, B.M. (2012). Emergence of embryonic pattern through contact inhibition of locomotion. Development 139, 4555-4560.

Deblandre, G.A., Wettstein, D.A., Koyano-Nakagawa, N., and Kintner, C. (1999) . A two-step mechanism generates the spacing pattern of the ciliated cells in the skin of Xenopus embryos. Development 126, 4715-4728.

Flanagan, J.G., Chan, D.C., and Leder, P. (1991). Transmembrane form of the kit ligand growth factor is determined by alternative splicing and is missing in the sld mutant. Cell 64, 1025-1035.

Fuerst, P.G., Koizumi, A., Masland, R.H., and Burgess, R.W. (2008). Neurite arborization and mosaic spacing in the mouse retina require DSCAM. Nature 451, $470-474$. 

dependent interactions position homotypic neurones in regular monolayered arrays during retinal development. Development 129, 3803-3814.

Goldman, D.C., Berg, L.K., Heinrich, M.C., and Christian, J.L. (2006) . Ectodermally derived steel/stem cell factor functions non-cell autonomously during primitive erythropoiesis in Xenopus. Blood 107, 3114-3121.

Gommerman, J.L., Sittaro, D., Klebasz, N.Z., Williams, D.A., and Berger, S.A. (2000). Differential stimulation of c-Kit mutants by membrane-bound and soluble Steel Factor correlates with leukemic potential. Blood 96, 3734-3742.

Gu, Y., Runyan, C., Shoemaker, A., Surani, A., and Wylie, C. (2009). Steel factor controls primordial germ cell survival and motility from the time of their specification in the allantois, and provides a continuous niche throughout their migration. Development 136, 1295-1303.

Gu, Y., Runyan, C., Shoemaker, A., Surani, M.A., and Wylie, C. (2011). Membrane-bound steel factor maintains a high local concentration for mouse primordial germ cell motility, and defines the region of their migration. PLoS One 6, e25984.

Haas, M., Gomez Vazquez, J.L., Sun, D.I., Tran, H.T., Brislinger, M., Tasca, A., Shomroni, O., Vleminckx, K., and Walentek, P. (2019). DeltaN-Tp63 Mediates Wnt/beta-Catenin-Induced Inhibition of Differentiation in Basal Stem Cells of Mucociliary Epithelia. Cell Rep 28, 3338-3352 e3336.

Hayes, J.M., Kim, S.K., Abitua, P.B., Park, T.J., Herrington, E.R., Kitayama, A., Grow, M.W., Ueno, N., and Wallingford, J.B. (2007). Identification of novel ciliogenesis factors using a new in vivo model for mucociliary epithelial development. Dev Biol 312, 115-130.

Heissig, B., Hattori, K., Dias, S., Friedrich, M., Ferris, B., Hackett, N.R., Crystal, R.G., Besmer, P., Lyden, D., Moore, M.A., et al. (2002). Recruitment of stem and progenitor cells from the bone marrow niche requires MMP-9 mediated release of kit-ligand. Cell 109, 625-637.

Hetrick, B., Han, M.S., Helgeson, L.A., and Nolen, B.J. (2013). Small molecules CK-666 and CK-869 inhibit actin-related protein 2/3 complex by blocking an activating conformational change. Chem Biol 20, 701-712.

Higashi, T., Arnold, T.R., Stephenson, R.E., Dinshaw, K.M., and Miller, A.L. (2016). Maintenance of the Epithelial Barrier and Remodeling of Cell-Cell Junctions during Cytokinesis. Curr Biol 26, 1829-1842.

Hu-Lowe, D.D., Zou, H.Y., Grazzini, M.L., Hallin, M.E., Wickman, G.R., Amundson, K., Chen, J.H., Rewolinski, D.A., Yamazaki, S., Wu, E.Y., et al. (2008). Nonclinical antiangiogenesis and antitumor activities of axitinib (AG013736), an oral, potent, and selective inhibitor of vascular endothelial growth factor receptor tyrosine kinases 1, 2, 3. Clin Cancer Res 14, 7272-7283.

Huang, Y.L., and Niehrs, C. (2014). Polarized Wnt signaling regulates ectodermal cell fate in Xenopus. Dev Cell 29, 250-257.

Hultman, K.A., Bahary, N., Zon, L.I., and Johnson, S.L. (2007). Gene Duplication of the zebrafish kit ligand and partitioning of melanocyte development functions to kit ligand a. PLoS Genet 3, el7.

Ing-Esteves, S., Kostadinov, D., Marocha, J., Sing, A.D., Joseph, K.S., Laboulaye, M.A., Sanes, J.R., and Lefebvre, J.L. (2018). Combinatorial Effects of Alpha- and Gamma-Protocadherins on Neuronal Survival and Dendritic SelfAvoidance. J Neurosci 38, 2713-2729.

Irion, U., Frohnhofer, H.G., Krauss, J., Colak Champollion, T., Maischein, H.M., Geiger-Rudolph, S., Weiler, C., and Nusslein-Volhard, C. (2014). Gap junctions composed of connexins 41.8 and 39.4 are essential for colour pattern formation in zebrafish. Elife 3, e05125.

Iwashita, M., Watanabe, M., Ishii, M., Chen, T., Johnson, S.L., Kurachi, Y., Okada, N., and Kondo, S. (2006). Pigment pattern in jaguar/obelix zebrafish is caused by a Kir7.1 mutation: implications for the regulation of melanosome movement. PLoS Genet 2, e197. 
Kaltenbach, S.L., Yu, J.K., and Holland, N.D. (2009). The origin and migration of the earliest-developing sensory neurons in the peripheral nervous system of amphioxus. Evol Dev 11, 142-151.

Kim, K., Lake, B.B., Haremaki, T., Weinstein, D.C., and Sokol, S.Y. (2012). Rab11 regulates planar polarity and migratory behavior of multiciliated cells in Xenopus embryonic epidermis. Dev Dyn 241, 1385-1395.

Kim, S., Ma, L., Shokhirev, M.N., Quigley, I., and Kintner, C. (2018). Multicilin and activated E2f4 induce multiciliated cell differentiation in primary fibroblasts. Sci Rep 8, 12369.

Lennartsson, J., and Ronnstrand, L. (2012). Stem cell factor receptor/c-Kit: from basic science to clinical implications. Physiol Rev 92, 1619-1649.

Lucas, B.D.K., T. (1981). An iterative image registration technique with application to stereo vision. Proceedings of Imaging Understanding Workshop.

Ma, L., Quigley, I., Omran, H., and Kintner, C. (2014). Multicilin drives centriole biogenesis via E2f proteins. Genes Dev 28, 1461-1471.

Mani, M., Venkatasubrahmanyam, S., Sanyal, M., Levy, S., Butte, A., Weinberg, K., and Jahn, T. (2009). Wiskott-Aldrich syndrome protein is an effector of Kit signaling. Blood 114, 2900-2908.

Marchal, L., Luxardi, G., Thome, V., and Kodjabachian, L. (2009). BMP inhibition initiates neural induction via FGF signaling and Zic genes. Proc Natl Acad Sci U S A 106, 17437-17442.

Martin, B.L., and Harland, R.M. (2004). The developmental expression of two Xenopus laevis steel homologues, Xsl-1 and Xsl-2. Gene Expr Patterns 5, 239243.

Meininger, C.J., Yano, H., Rottapel, R., Bernstein, A., Zsebo, K.M., and zetter, B.R. (1992). The c-kit receptor ligand functions as a mast cell chemoattractant. Blood 79, 958-963.

Miyazawa, K., Williams, D.A., Gotoh, A., Nishimaki, J., Broxmeyer, H.E., and Toyama, K. (1995). Membrane-bound Steel factor induces more persistent tyrosine kinase activation and longer life span of c-kit gene-encoded protein than its soluble form. Blood 85, 641-649.

Quigley, I.K., Stubbs, J.L., and Kintner, C. (2011). Specification of ion transport cells in the Xenopus larval skin. Development 138, 705-714.

Rizvi, S.A., Neidt, E.M., Cui, J., Feiger, Z., Skau, C.T., Gardel, M.L., Kozmin, S.A., and Kovar, D.R. (2009). Identification and characterization of a small molecule inhibitor of formin-mediated actin assembly. Chem Biol 16, 11581168 .

Runyan, C., Schaible, K., Molyneaux, K., Wang, Z., Levin, L., and Wylie, C. (2006). Steel factor controls midline cell death of primordial germ cells and is essential for their normal proliferation and migration. Development 133, $4861-4869$.

Samayawardhena, L.A., Kapur, R., and Craig, A.W. (2007). Involvement of Fyn kinase in Kit and integrin-mediated Rac activation, cytoskeletal reorganization, and chemotaxis of mast cells. Blood 109, 3679-3686.

Sedzinski, J., Hannezo, E., Tu, F., Biro, M., and Wallingford, J.B. (2016) . Emergence of an Apical Epithelial Cell Surface In Vivo. Dev Cell 36, $24-35$.

Sedzinski, J., Hannezo, E., Tu, F., Biro, M., and Wallingford, J.B. (2017) . RhoA regulates actin network dynamics during apical surface emergence in multiciliated epithelial cells. J Cell Sci 130, 420-428.

Sirour, C., Hidalgo, M., Bello, V., Buisson, N., Darribere, T., and Moreau, N. (2011). Dystroglycan is involved in skin morphogenesis downstream of the Notch signaling pathway. Mol Biol Cell 22, 2957-2969. 
Stramer, B., and Mayor, R. (2016). Mechanisms and in vivo functions of contact inhibition of locomotion. Nat Rev Mol Cell Biol.

Stubbs, J.L., Davidson, L., Keller, R., and Kintner, C. (2006). Radial intercalation of ciliated cells during Xenopus skin development. Development 133, 2507-2515.

Stubbs, J.L., Vladar, E.K., Axelrod, J.D., and Kintner, C. (2012). Multicilin promotes centriole assembly and ciliogenesis during multiciliate cell differentiation. Nat Cell Biol 14, 140-147.

Suraneni, P., Fogelson, B., Rubinstein, B., Noguera, P., Volkmann, N., Hanein, D., Mogilner, A., and Li, R. (2015). A mechanism of leading-edge protrusion in the absence of Arp2/3 complex. Mol Biol Cell 26, 901-912.

Suraneni, P., Rubinstein, B., Unruh, J.R., Durnin, M., Hanein, D., and Li, R. (2012). The Arp2/3 complex is required for lamellipodia extension and directional fibroblast cell migration. J Cell Biol 197, 239-251.

Tabone-Eglinger, S., Calderin-Sollet, Z., Pinon, P., Aebischer, N., WehrleHaller, M., Jacquier, M.C., Boettiger, D., and Wehrle-Haller, B. (2014). Niche anchorage and signaling through membrane-bound Kit-ligand/c-kit receptor are kinase independent and imatinib insensitive. FASEB J 28, 4441-4456.

Tabone-Eglinger, S., Wehrle-Haller, M., Aebischer, N., Jacquier, M.C., and Wehrle-Haller, B. (2012). Membrane-bound Kit ligand regulates melanocyte adhesion and survival, providing physical interaction with an intraepithelial niche. FASEB J 26, 3738-3753.

Tajima, Y., Moore, M.A., Soares, V., Ono, M., Kissel, H., and Besmer, P. (1998). Consequences of exclusive expression in vivo of Kit-ligand lacking the major proteolytic cleavage site. Proc Natl Acad Sci U S A 95, 11903-11908.

Trindade, M., Messenger, N., Papin, C., Grimmer, D., Fairclough, L., Tada, M., and Smith, J.C. (2003). Regulation of apoptosis in thexenopus embryo by Bix3. Development 130, 4611-4622.

Villar-Cervino, V., Molano-Mazon, M., Catchpole, T., Valdeolmillos, M., Henkemeyer, M., Martinez, L.M., Borrell, V., and Marin, O. (2013). Contact repulsion controls the dispersion and final distribution of Cajal-Retzius cells. Neuron 77, 457-471.

Walderich, B., Singh, A.P., Mahalwar, P., and Nusslein-Volhard, C. (2016). Homotypic cell competition regulates proliferation and tiling of zebrafish pigment cells during colour pattern formation. Nat Commun 7, 11462.

Wehrle-Haller, B., Meller, M., and Weston, J.A. (2001). Analysis of melanocyte precursors in Nfl mutants reveals that MGF/KIT signaling promotes directed cell migration independent of its function in cell survival. Dev Biol 232, $471-483$.

Wehrle-Haller, B., and Weston, J.A. (1995). Soluble and cell-bound forms of steel factor activity play distinct roles in melanocyte precursor dispersal and survival on the lateral neural crest migration pathway. Development 121, $731-$ 742 .

Werner, M.E., and Mitchell, B.J. (2012). Understanding ciliated epithelia: the power of Xenopus. Genesis 50, 176-185.

Werner, M.E., Mitchell, J.W., Putzbach, W., Bacon, E., Kim, S.K., and Mitchell, B.J. (2014). Radial intercalation is regulated by the Par complex and the microtubule-stabilizing protein CLAMP/Spef1. J Cell Biol 206, 367-376.

Zuo, K., Kuang, D., Wang, Y., Xia, Y., Tong, W., Wang, X., Chen, Y., Duan, Y., and Wang, G. (2016). SCF/C-kit transactivates CXCR4-serine 339 phosphorylation through G protein-coupled receptor kinase 6 and regulates cardiac stem cell migration. Sci Rep 6, 26812. 

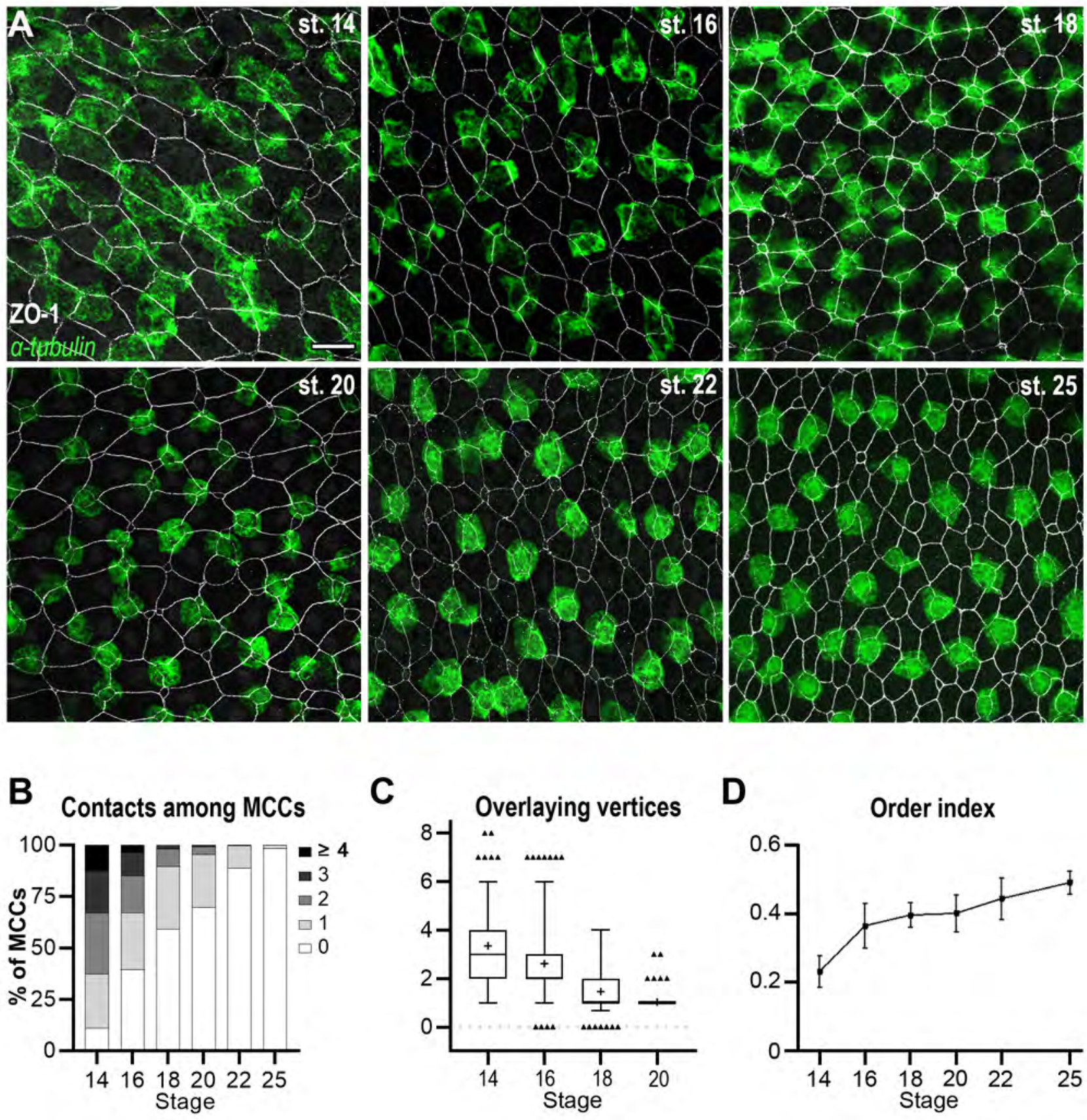

\section{Figure 1}



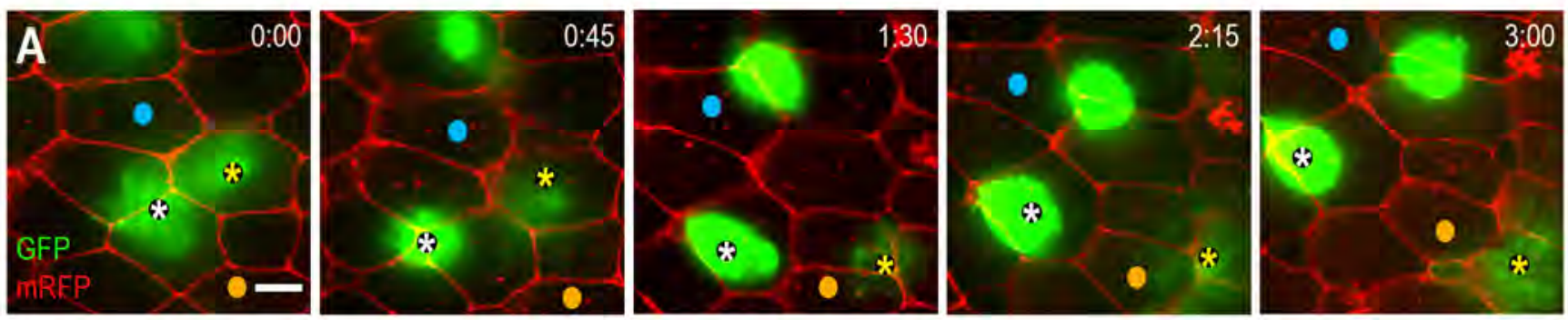

B
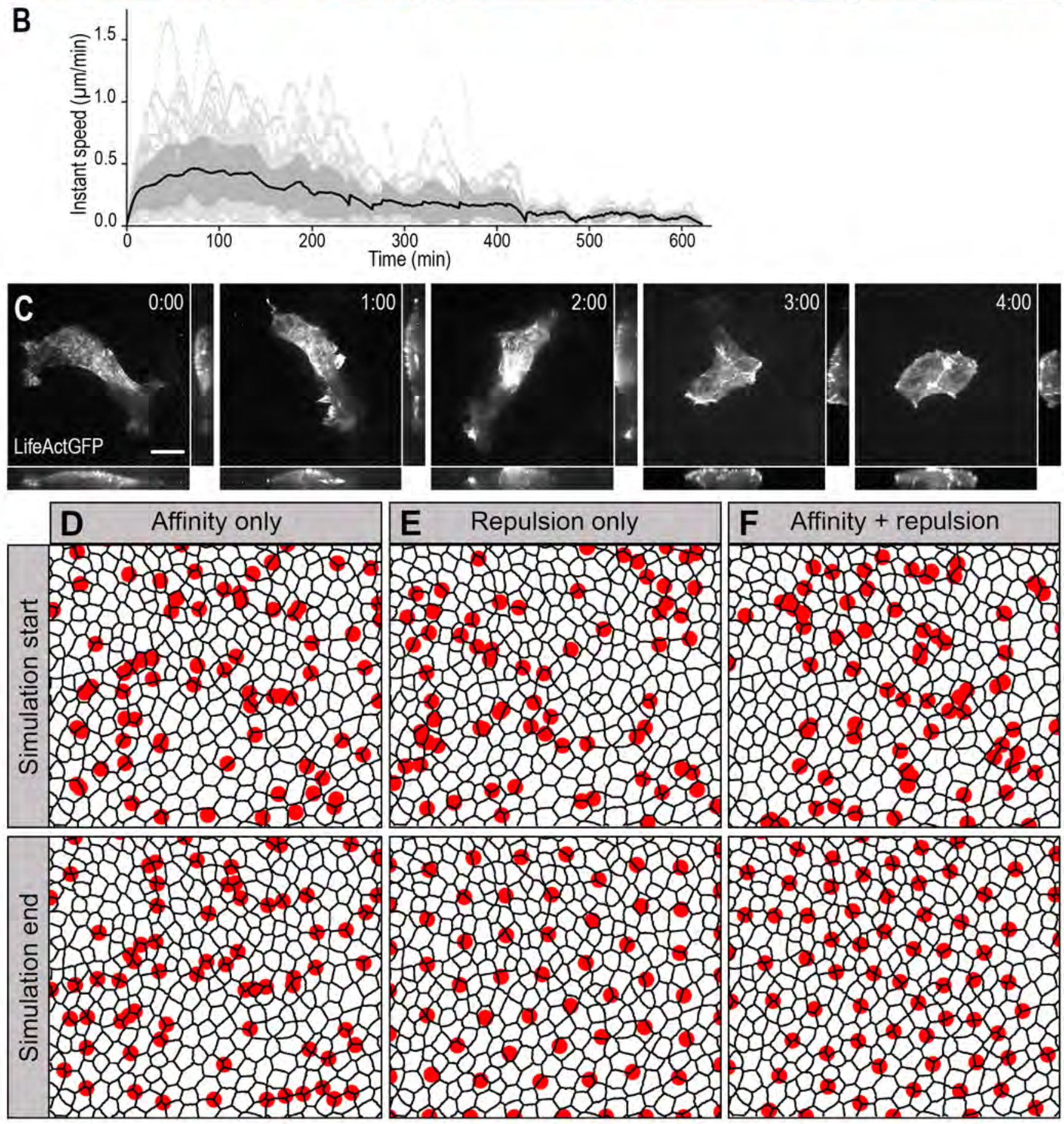

Figure 2 

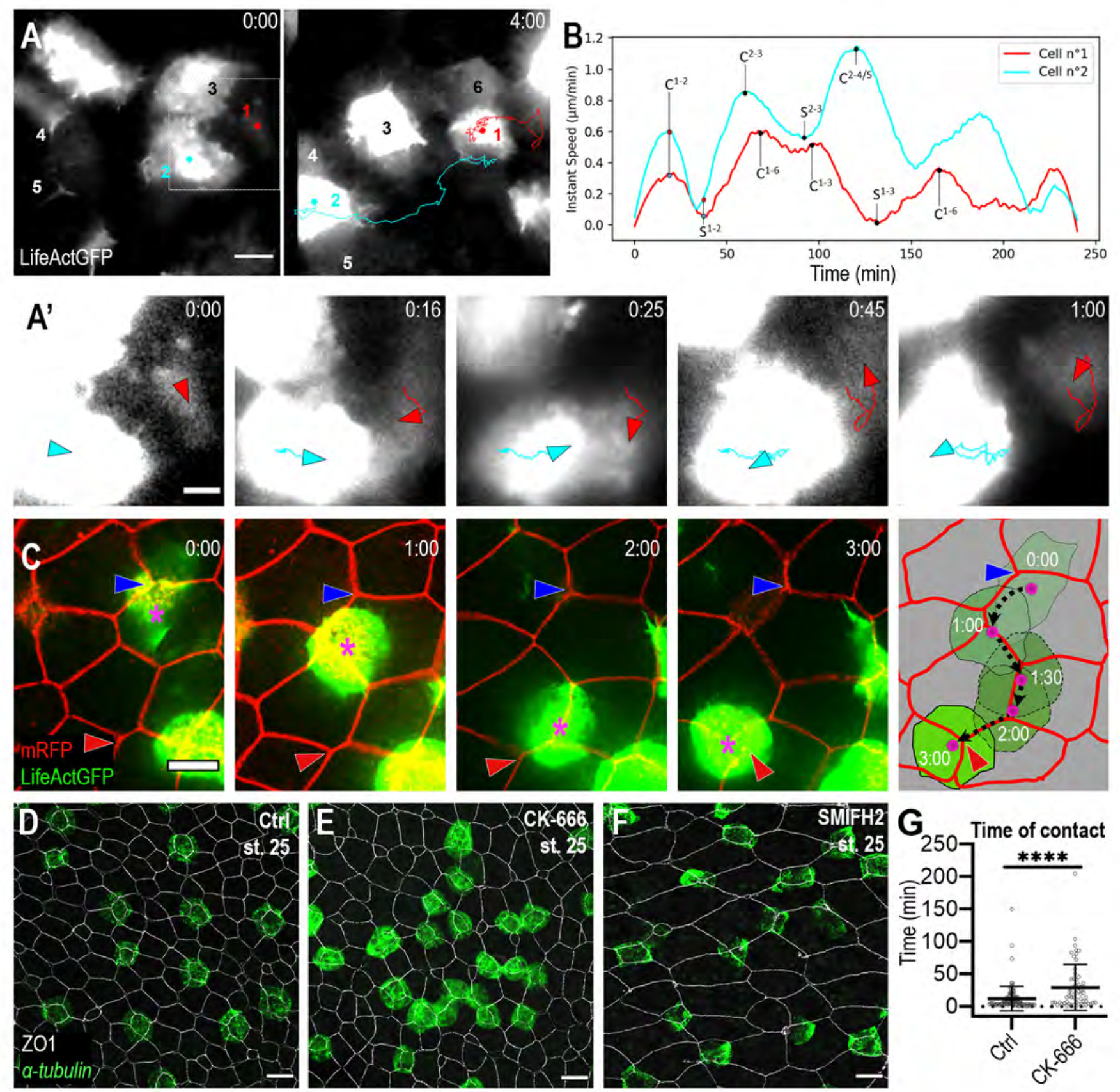

H

MCCs following junctions

Order index
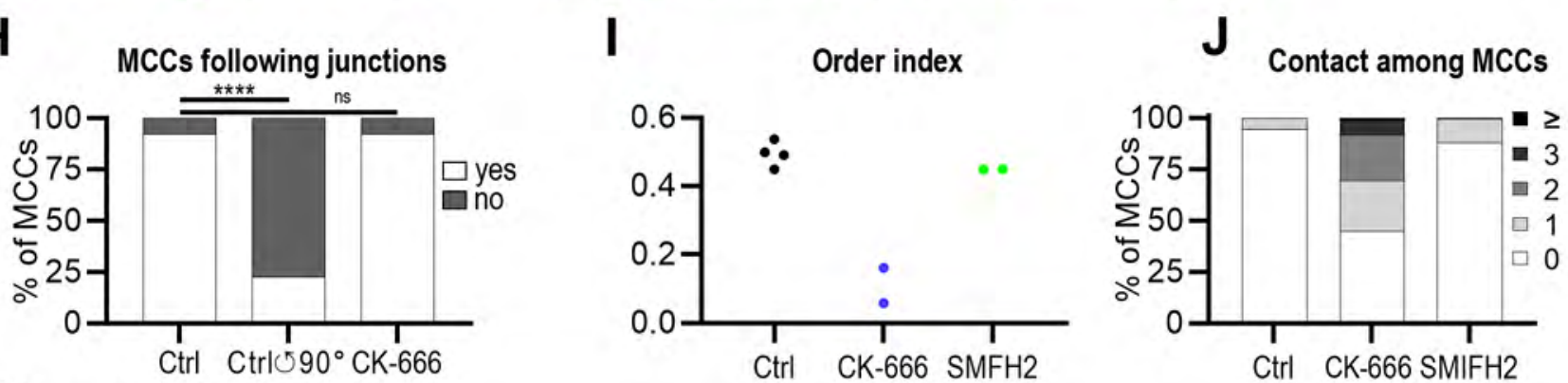

Figure 3
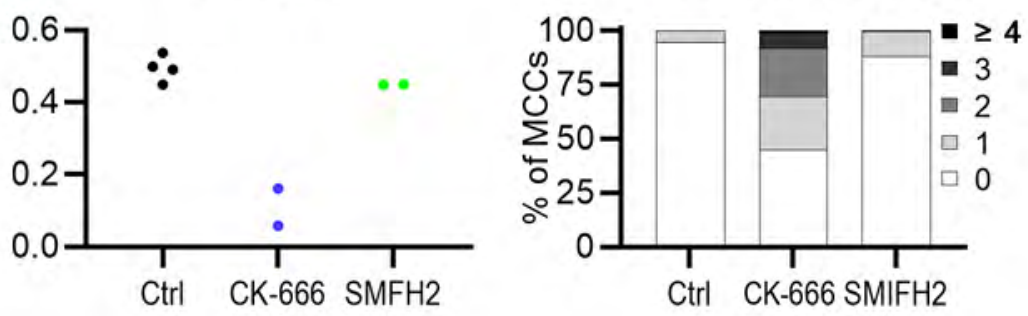

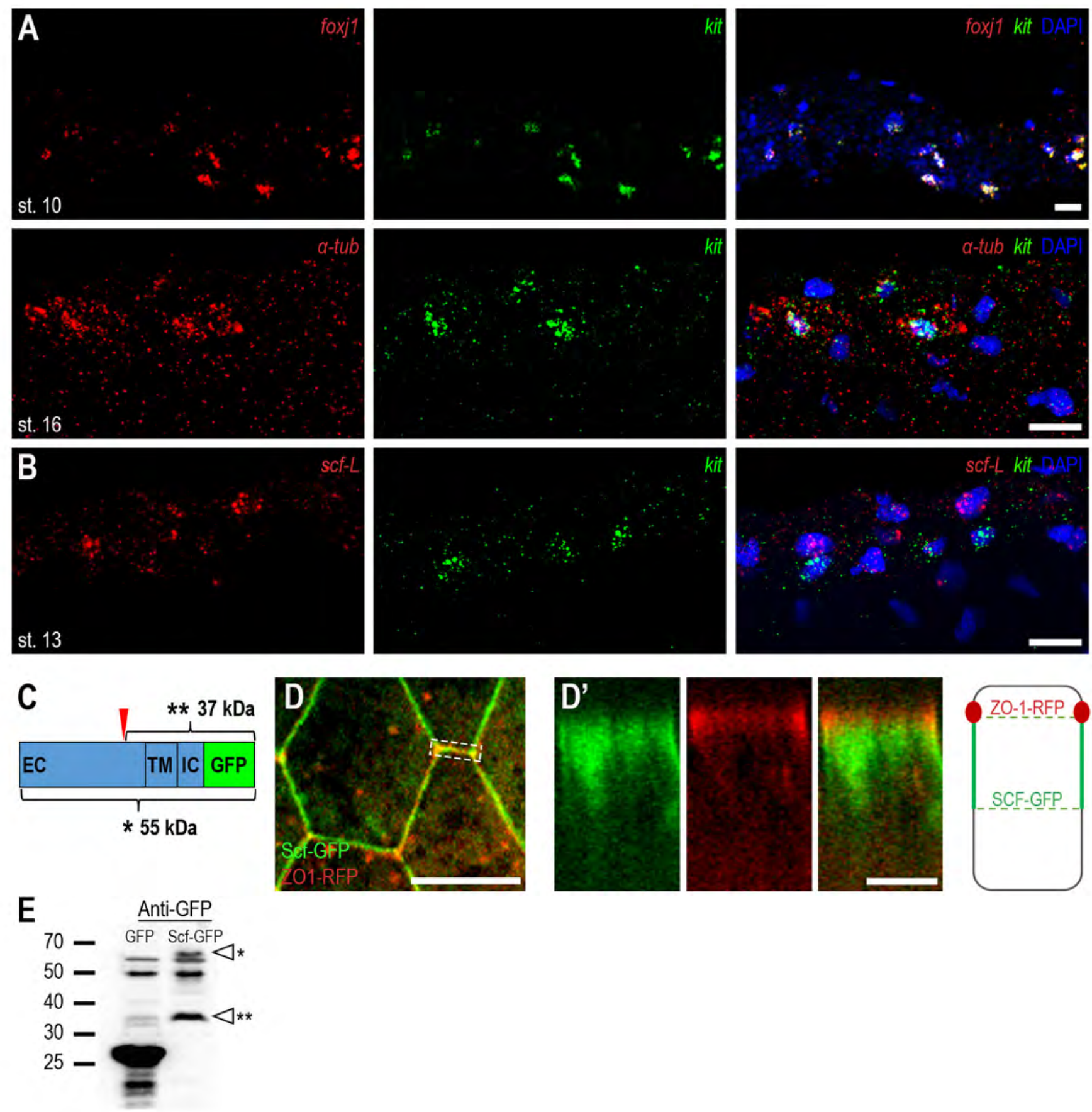

Figure 4 

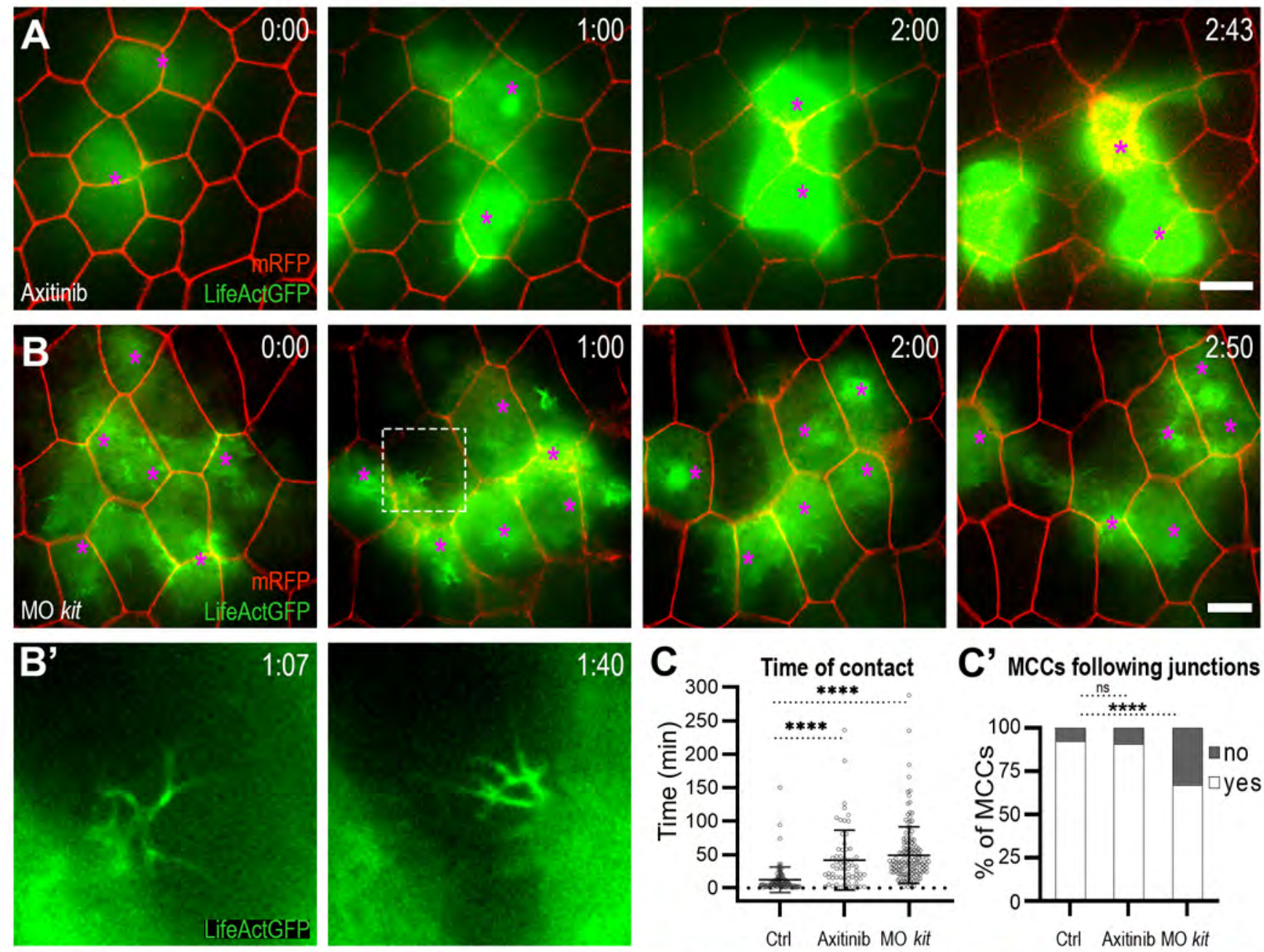

C' MCCs following junctions

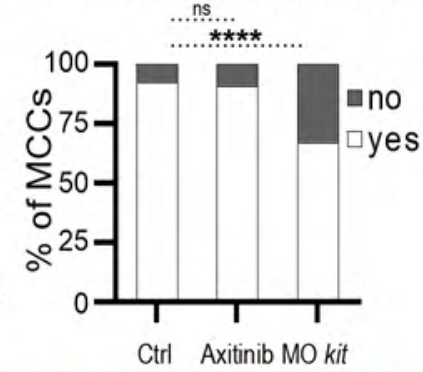

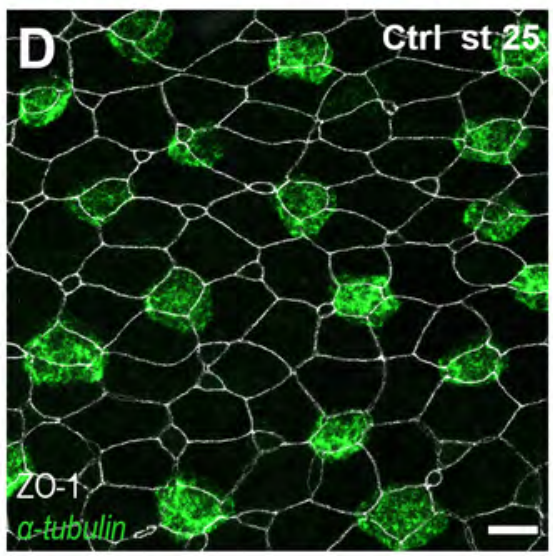

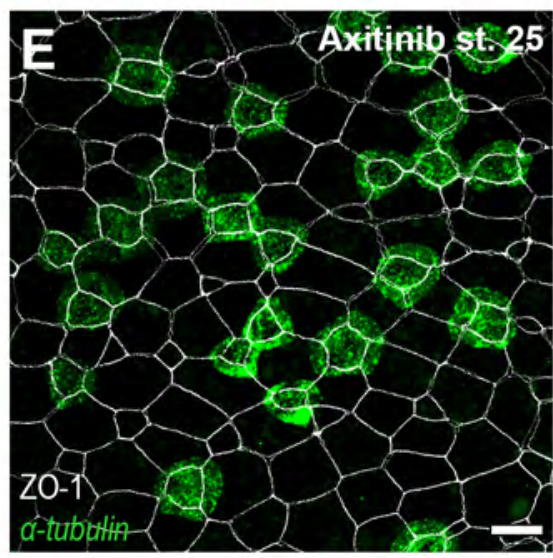

H

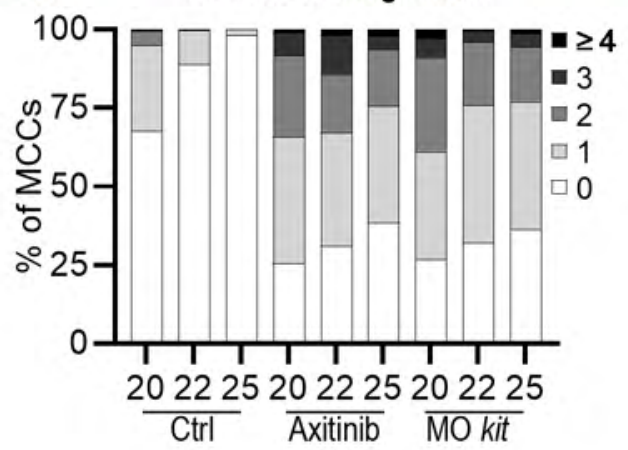

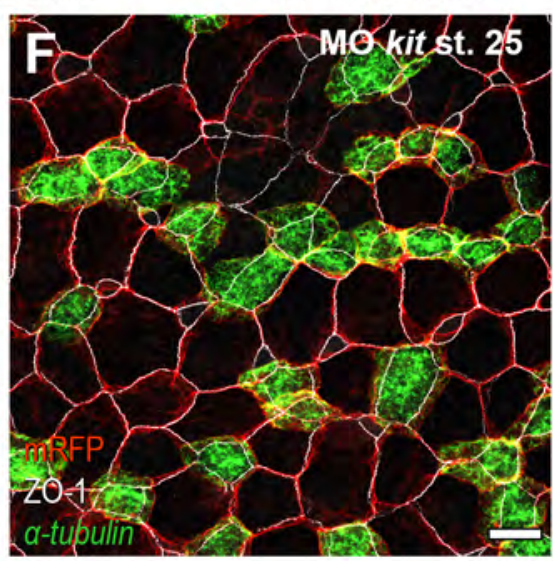

Order index

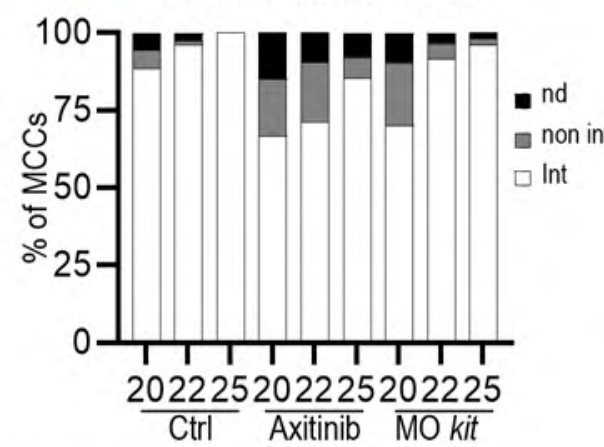

Figure 5 

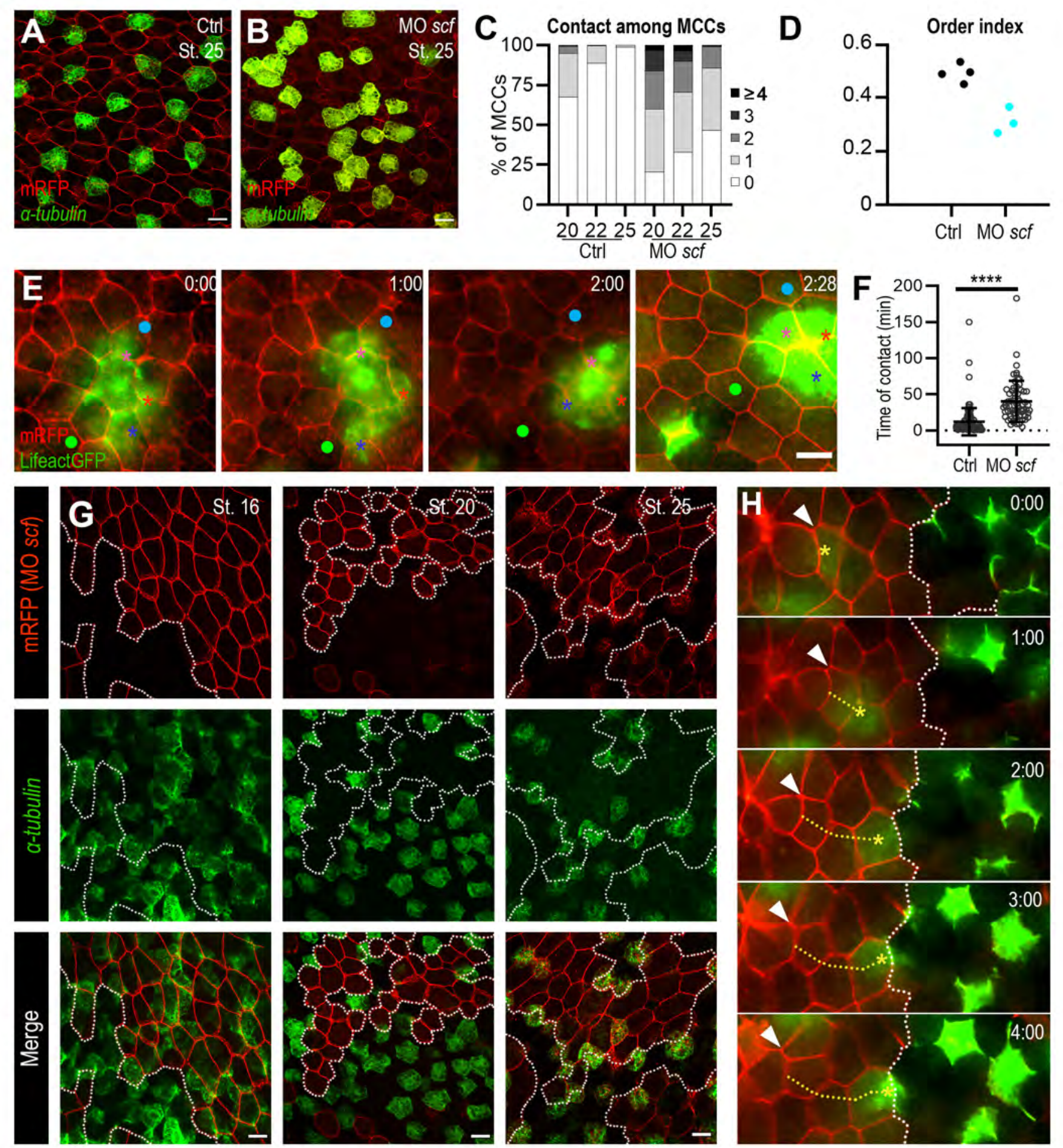

I MCC following Junctions
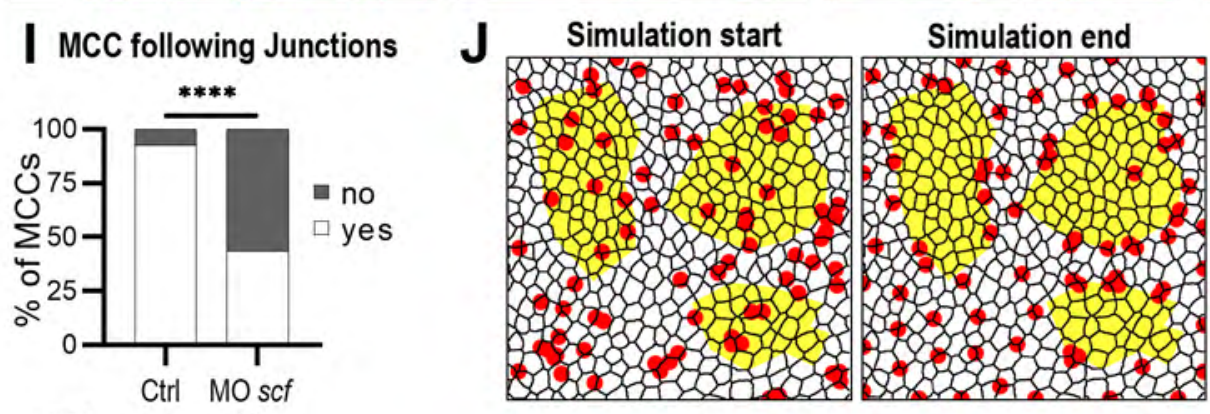

- MCCs

Low afffinity

+ low repulsion zones

Figure 6 

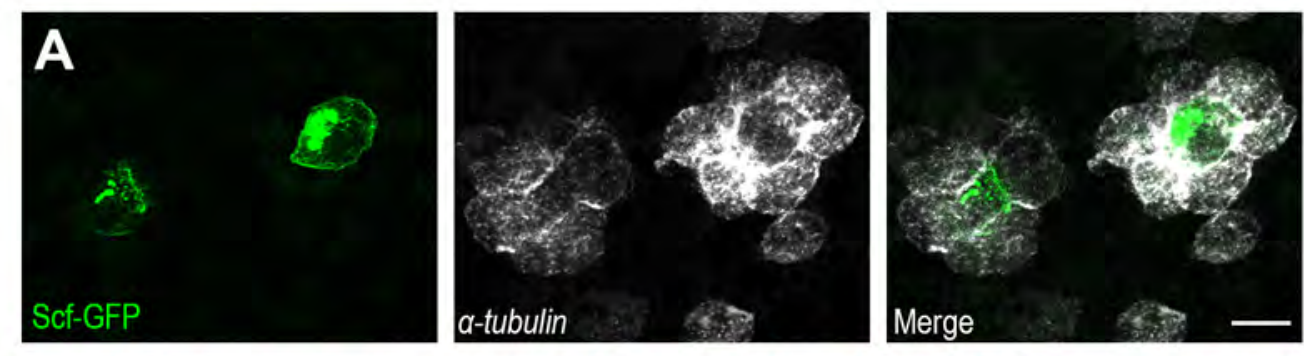

B Contact among MCCs
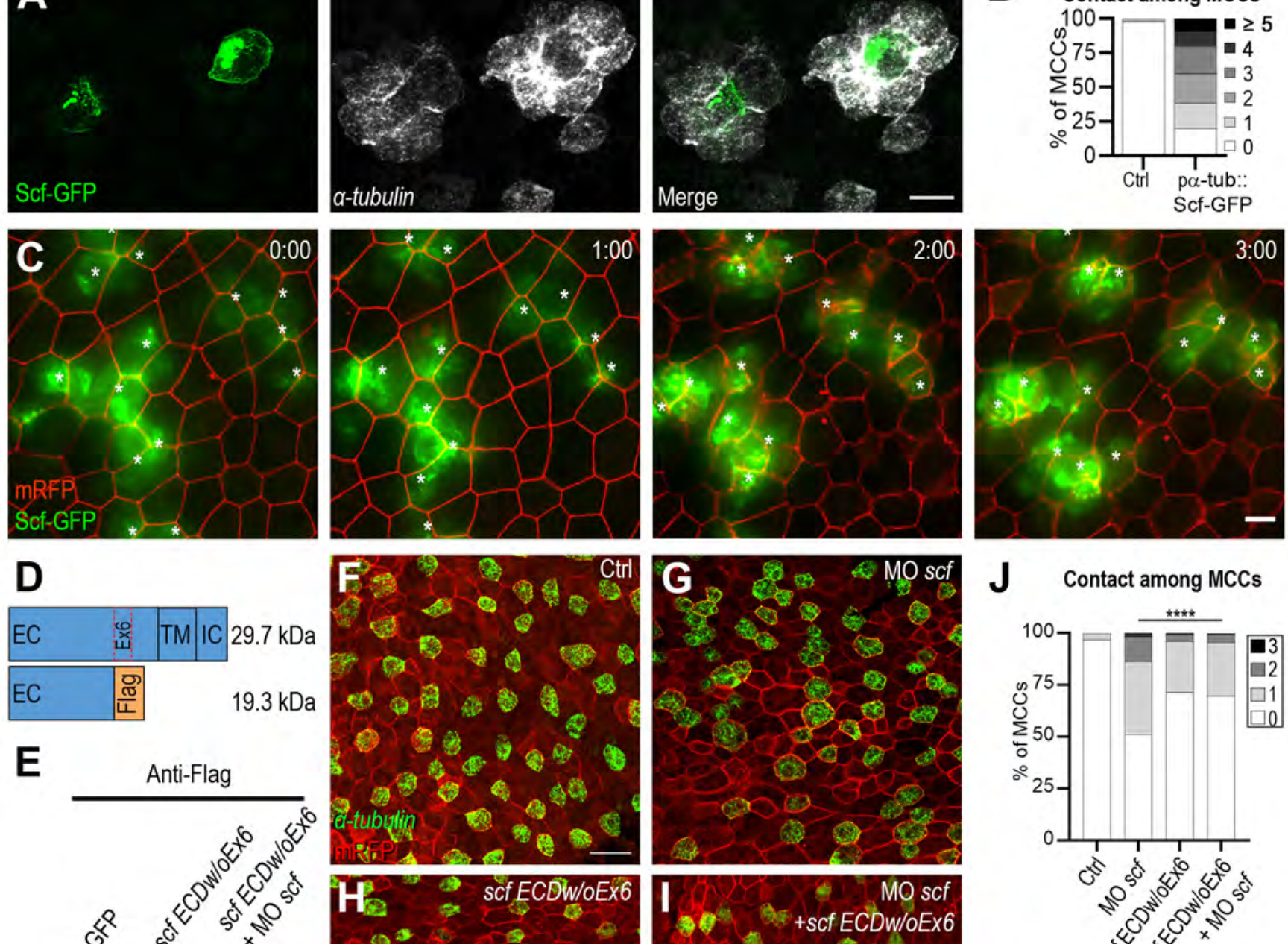

J Contact among MCCs
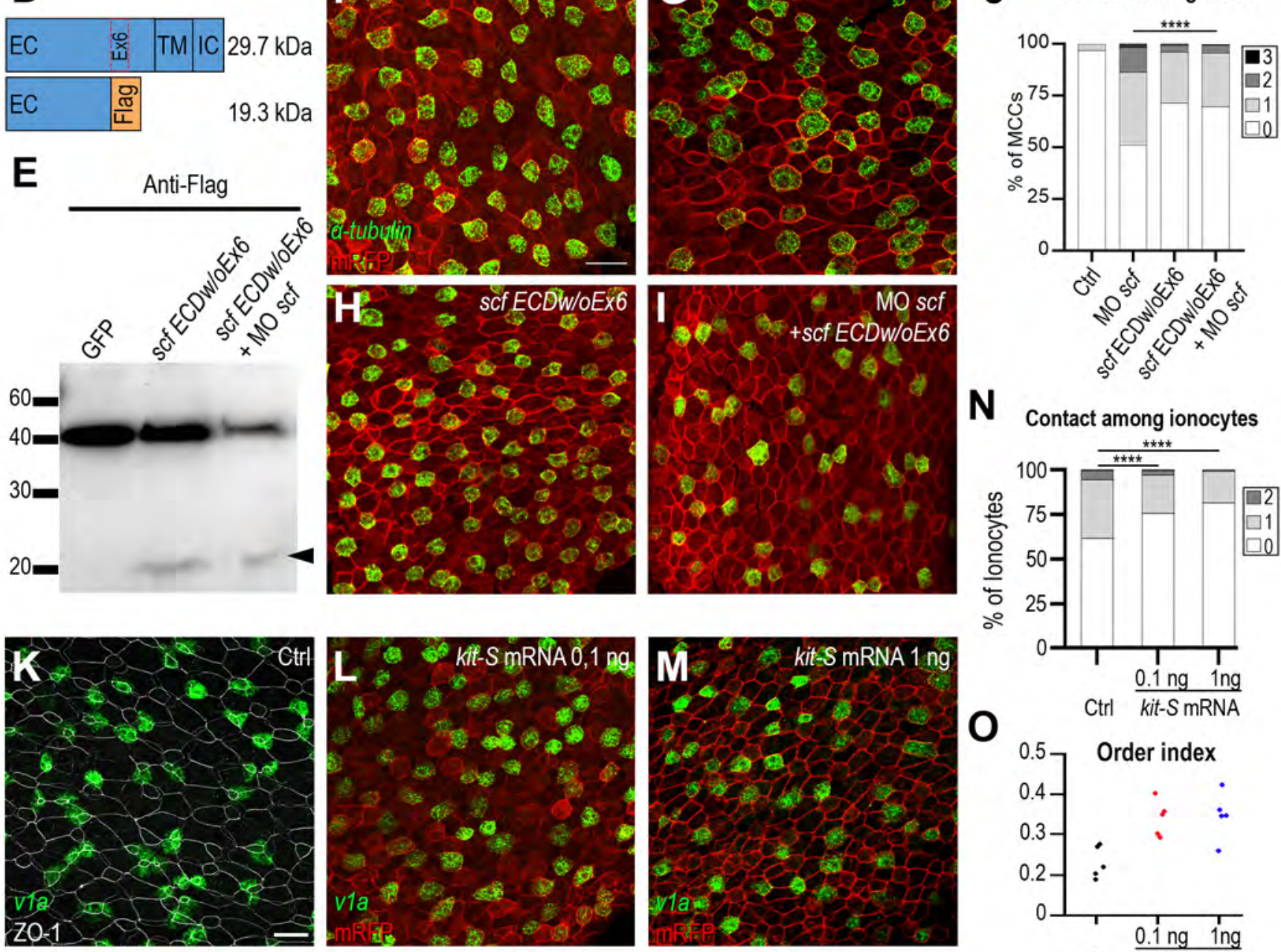

N Contact among ionocytes

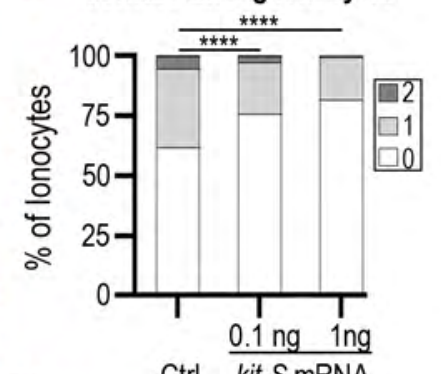

0

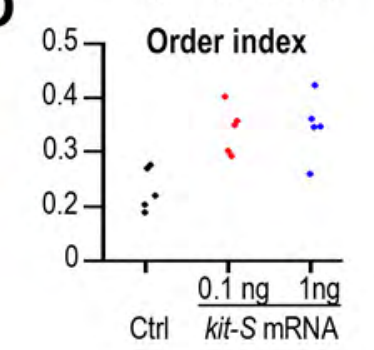

Figure 7 

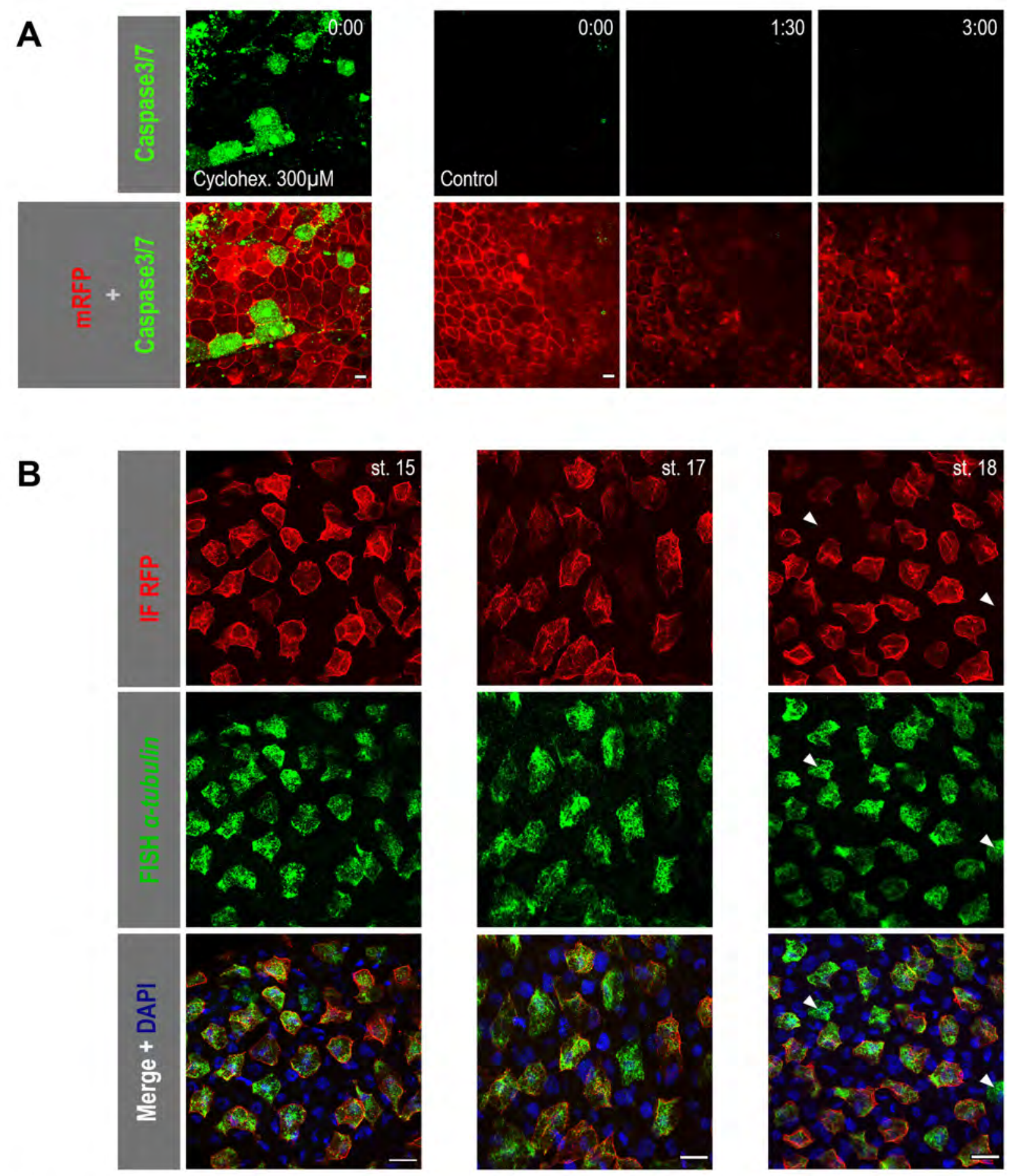

C

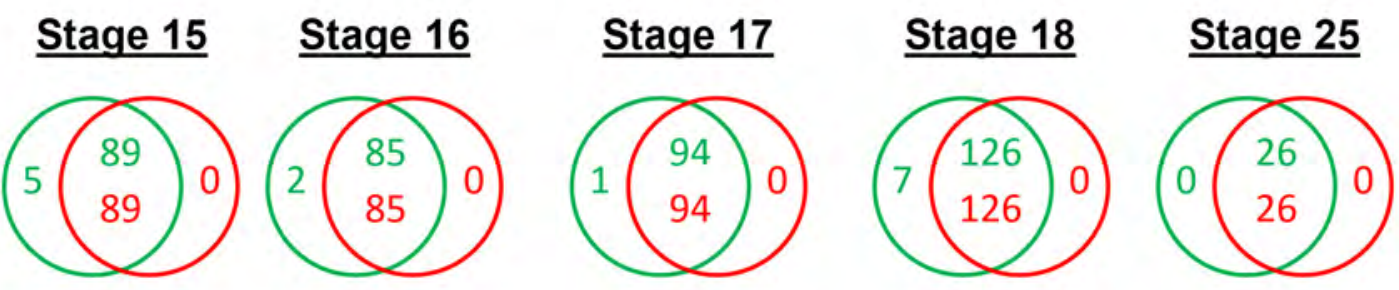

Figure S1 

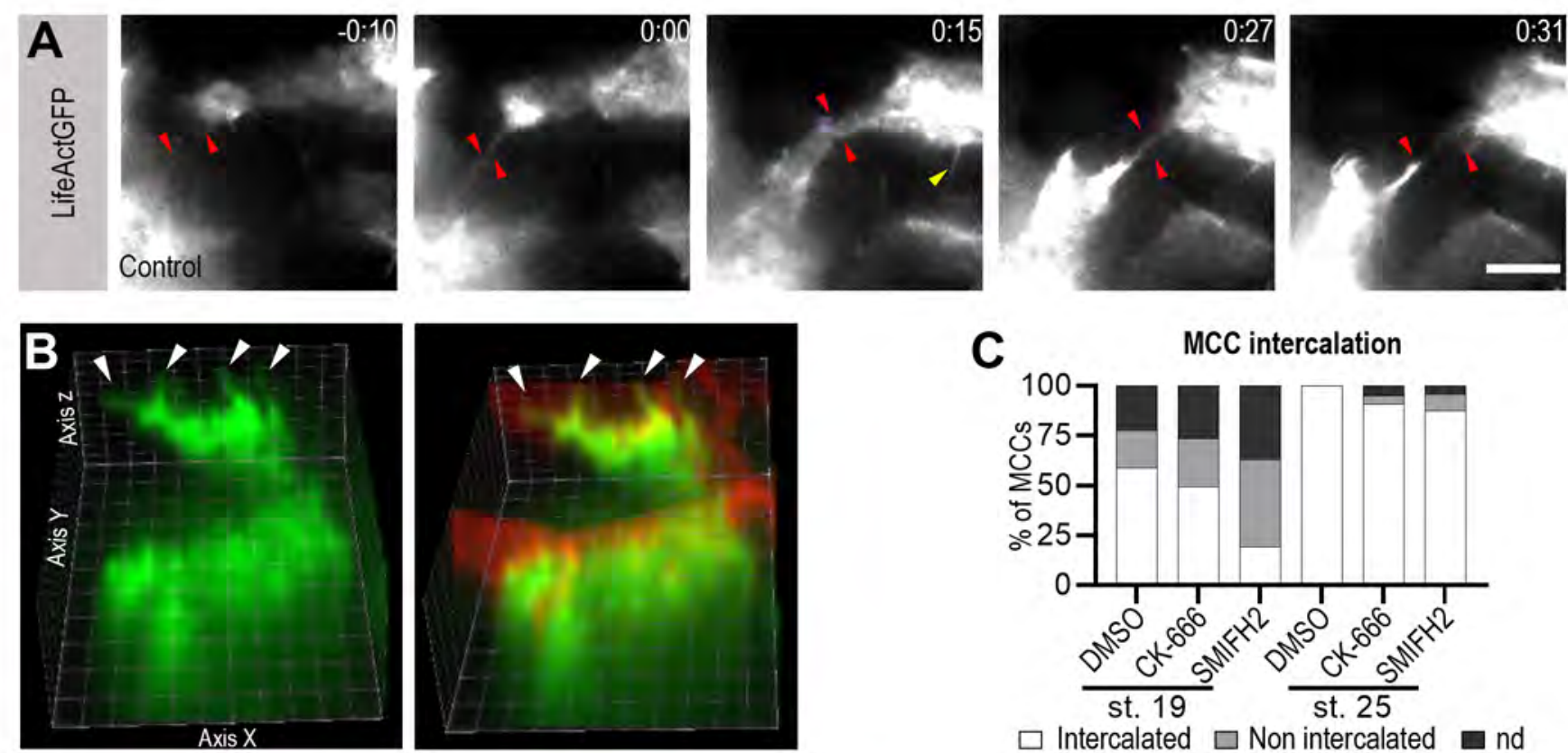

C
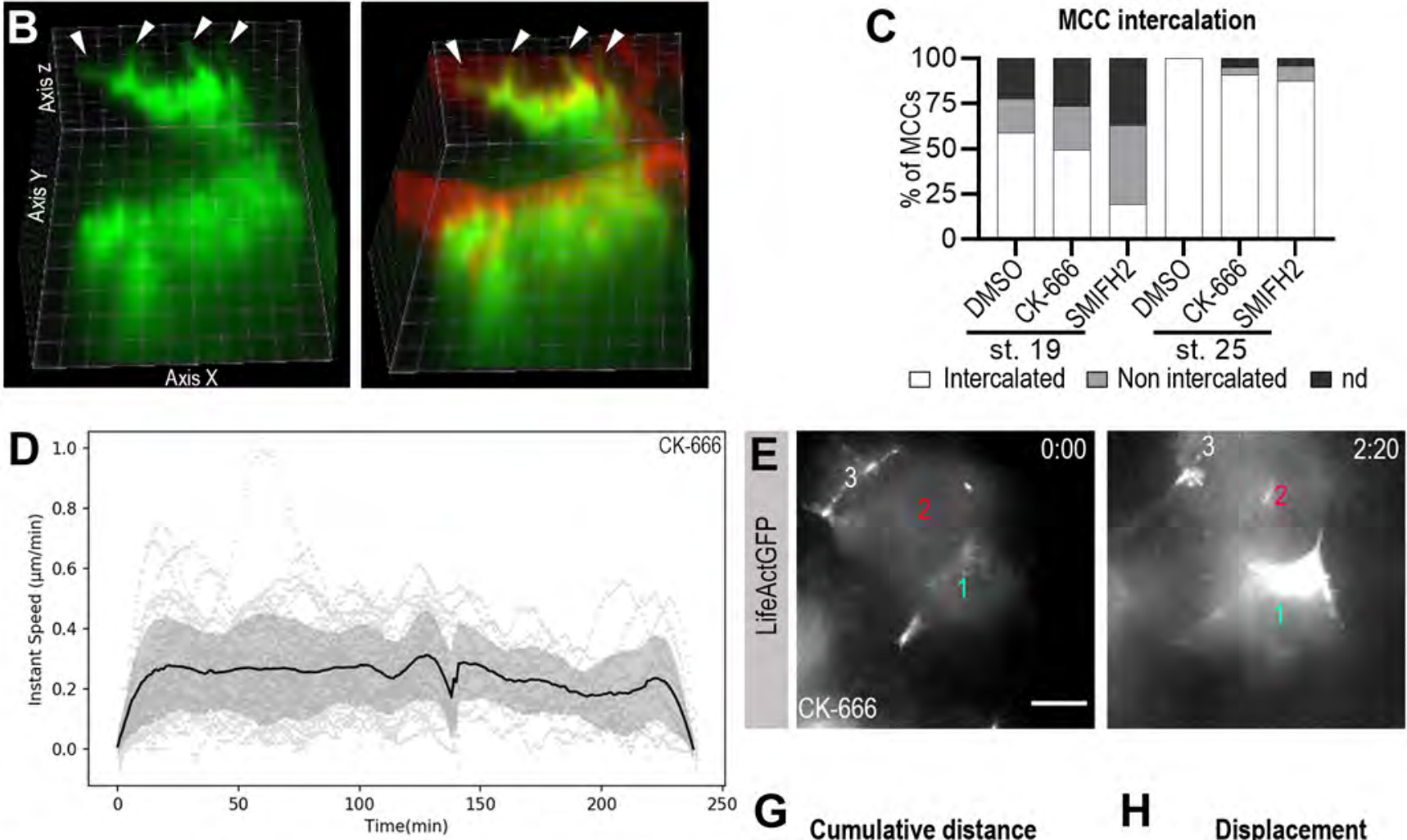

F
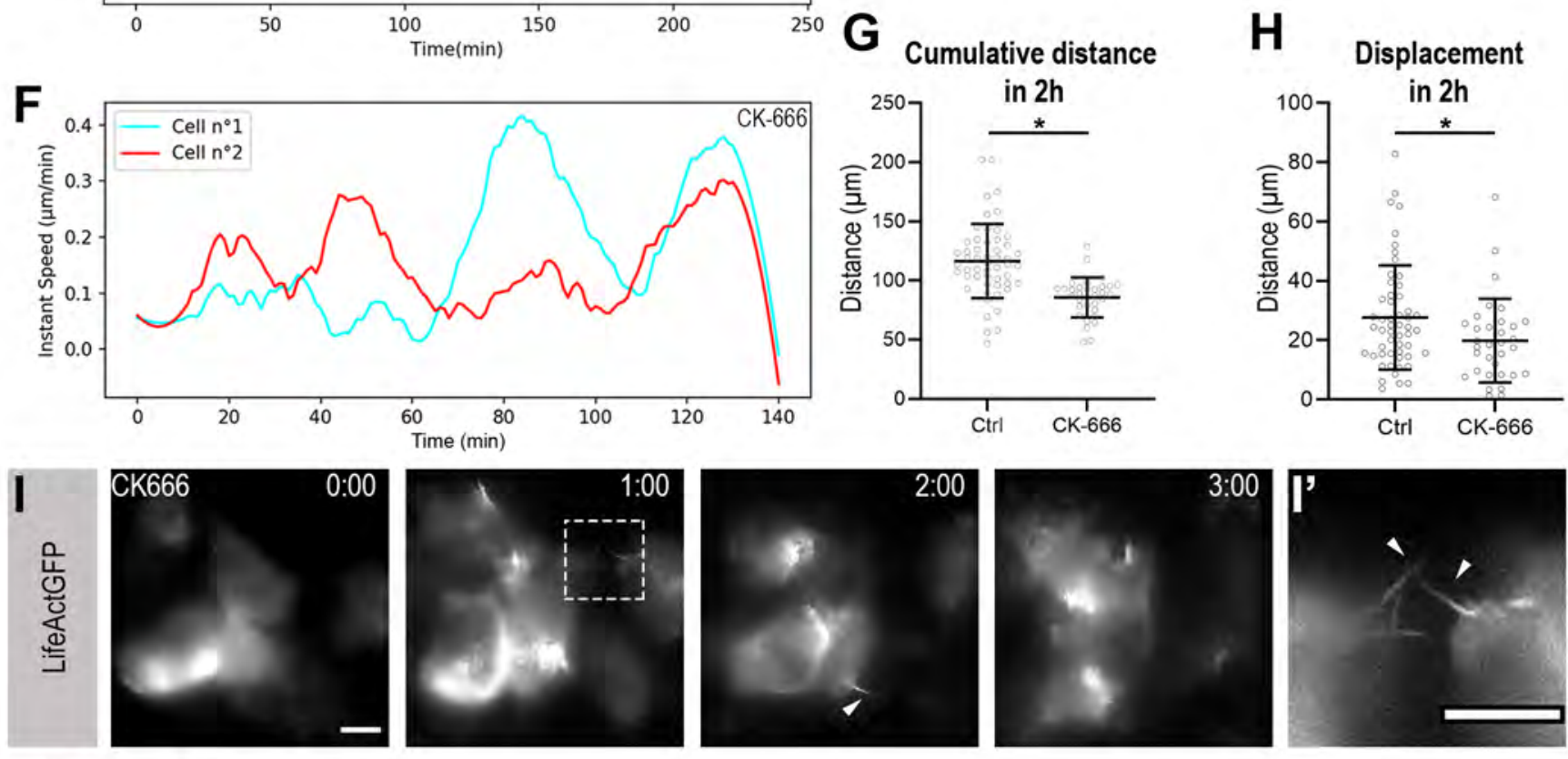

Figure S2 

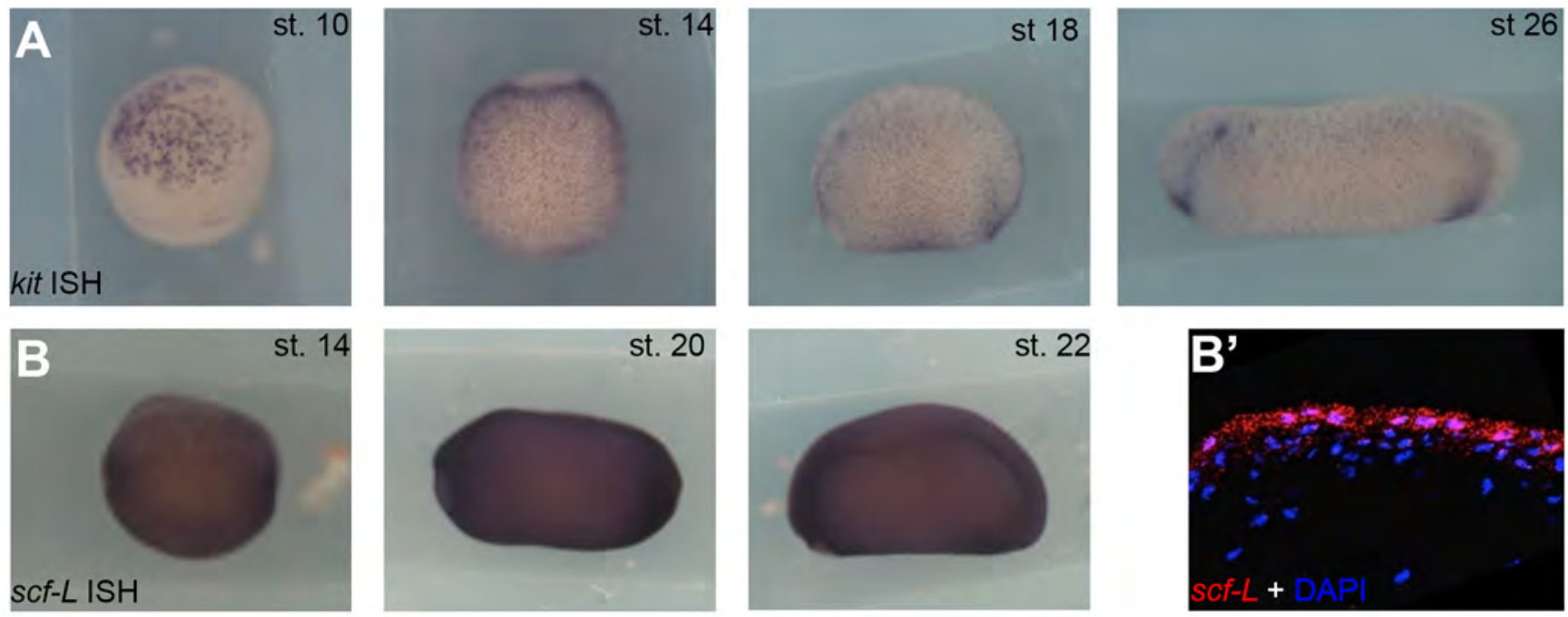

C

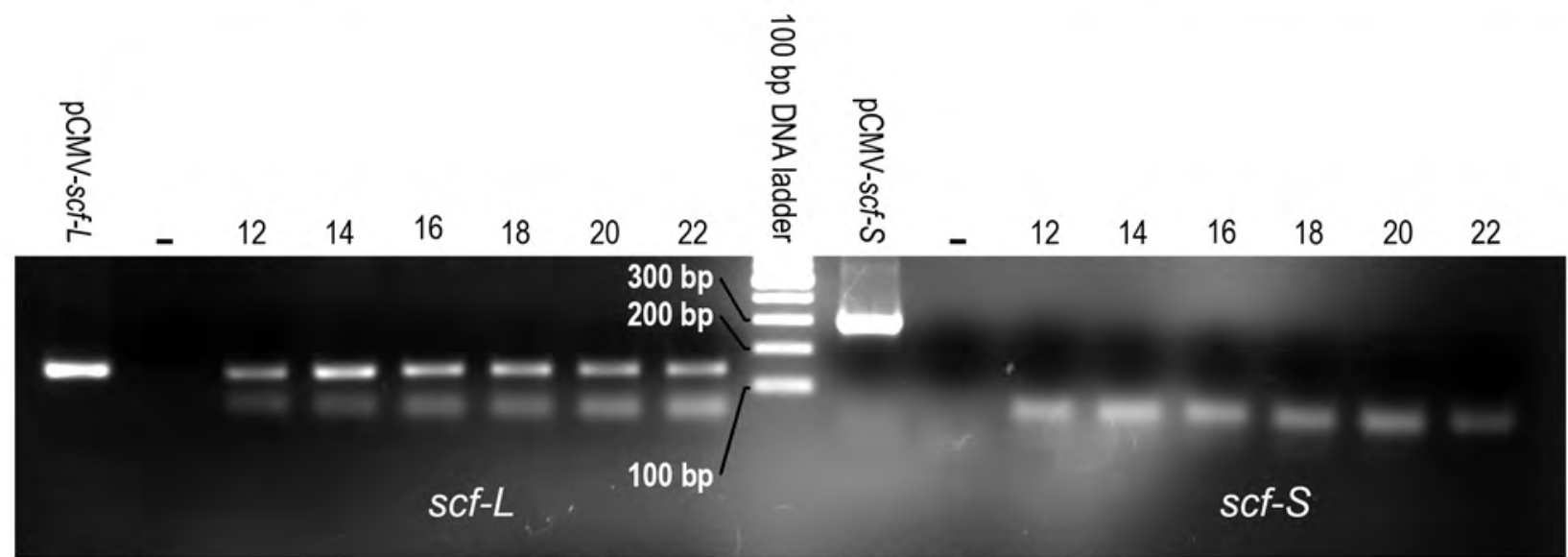

Figure S3

$2 \%(w / v)$ agarose gel in TAE, EtBr staining 
A

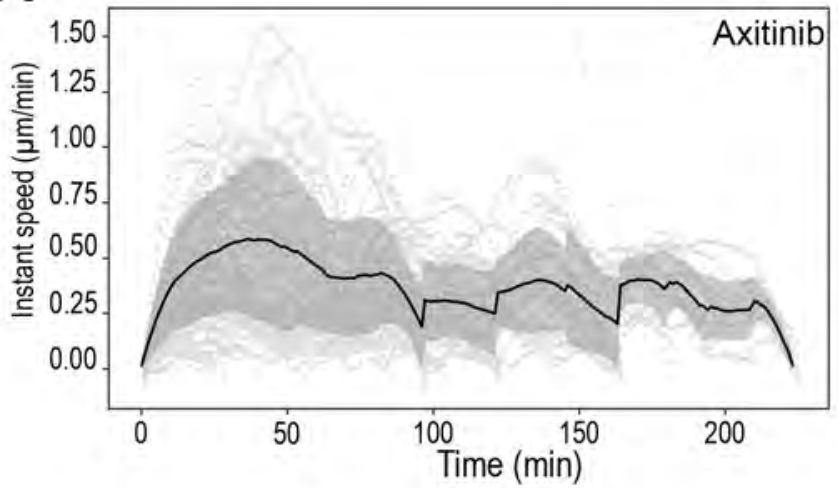

B

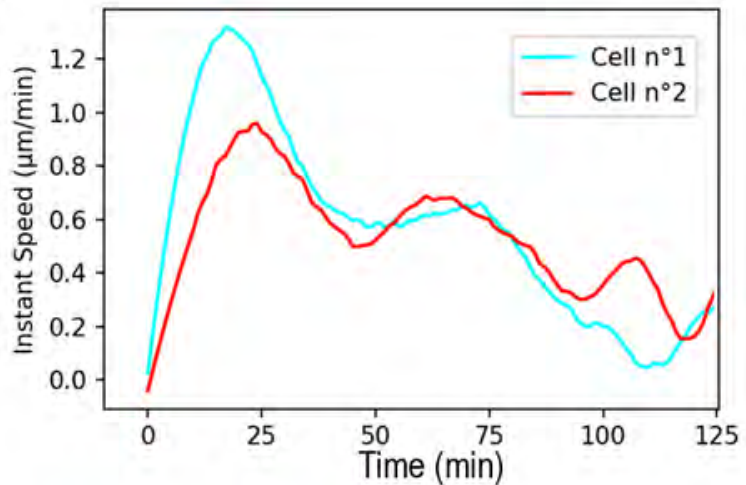

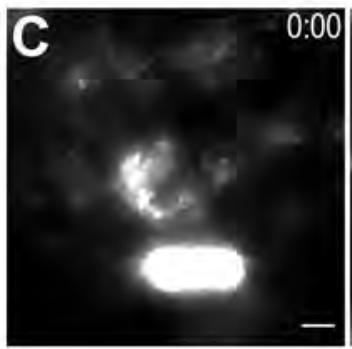
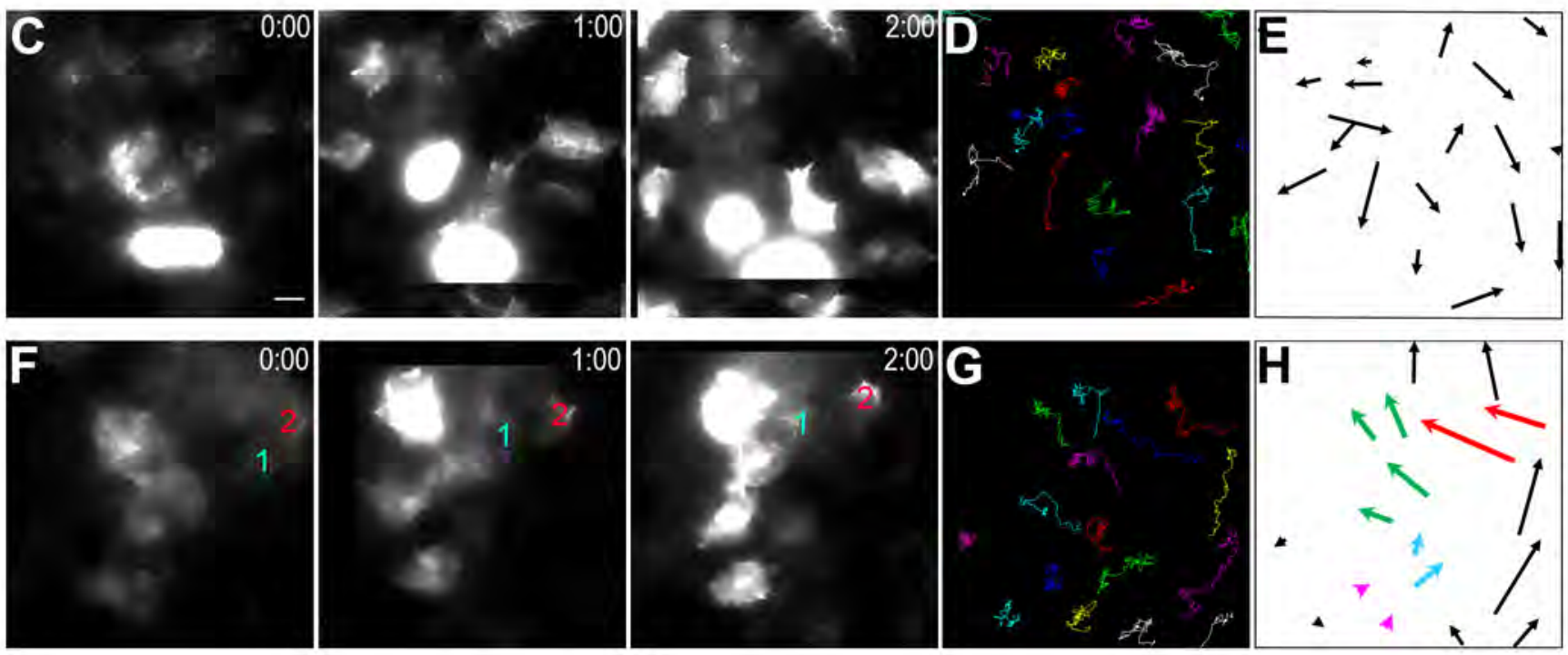

I

Cumulative distance in 2 hours
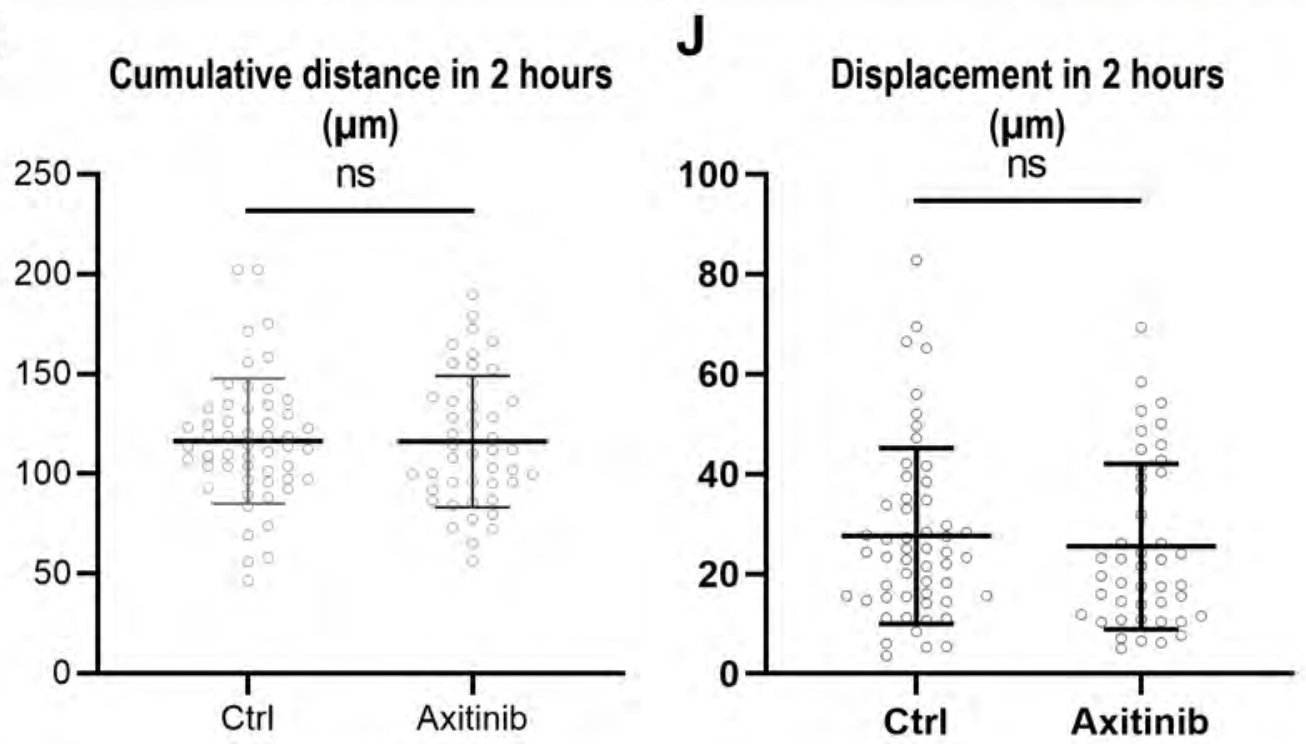

Figure S4 

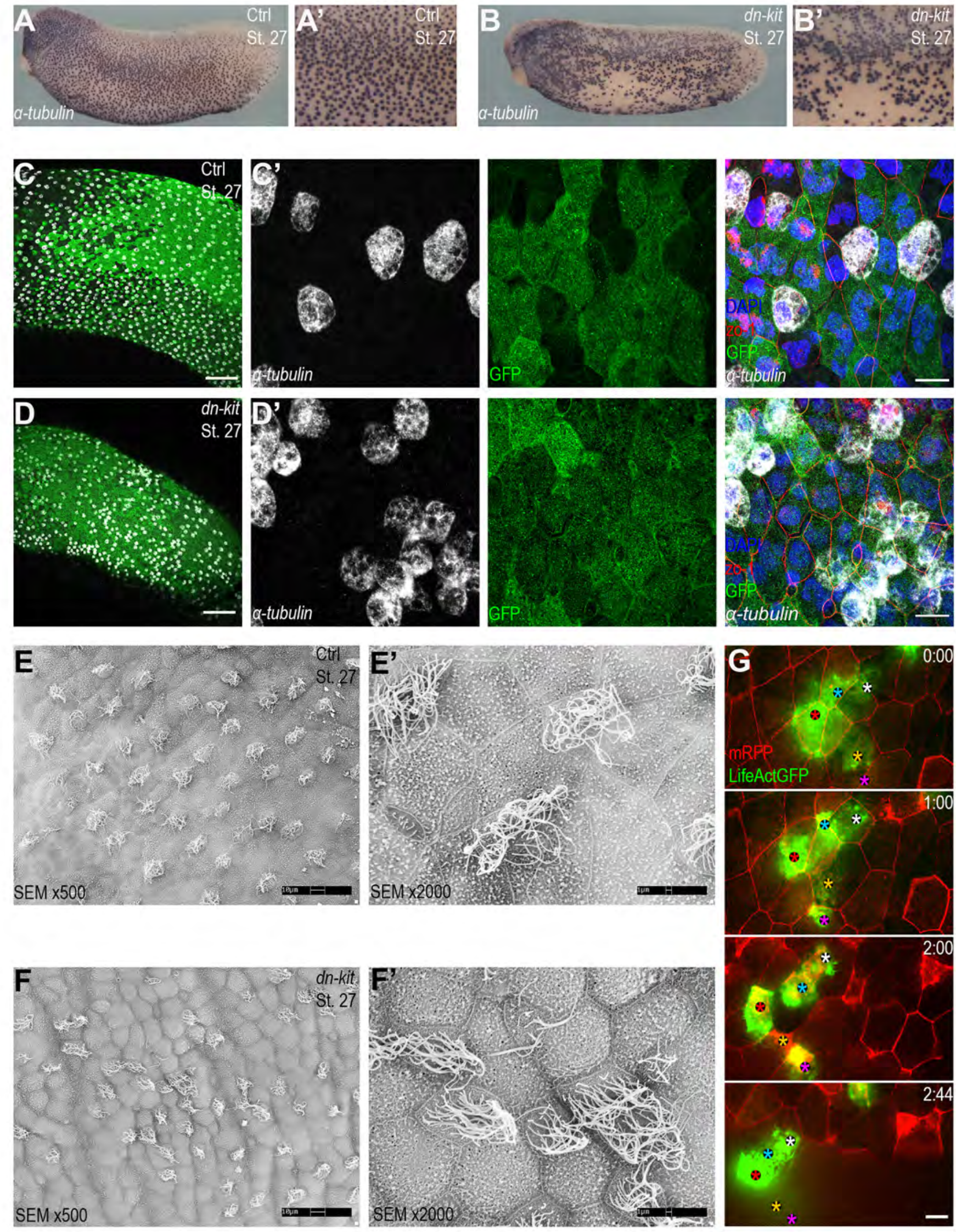

Figue S5 


\section{A 1) MO-ATG-kit-L}

kit-L

GAGGCTACAGCGCGGAGCTGCGCTACAATGAGCCACTTTCCATCCTTTACGATCACAATGTCACAATGTTACCTGTTGATGCTGCTTTTCCTTC ITCCTTACACAGGGGATGCAGTTCCT

2) MO-splice-kit-L

GGGATGCAGTTCCTAAAATTAATGACGGGGAAGACAGAGTCACTGTGAATGTTGGTGATAAGGTTTCGTTGGATGCAGAGATGTTCAG ITAGTTAAATTGGCTTTTCAGAAGTTGGGCCCTCTGAAGAAACCTCGAGATTTGAAAAGCCGTTCATATAACAAAAGCAAAACTGATCA GATCATAGTCATTGCTAAAGCAGATCTCAAGCACGTCGGAAGATATATATGCACAAATACAGAGACGCAAGAAAATACCTCAGTCTCTCT CTTTGTGAAGG GTAAGTCATTCATTGGGTTCCATGTCAAAGATCA

\section{B 1) MO-ATG-kit-S kit-S}

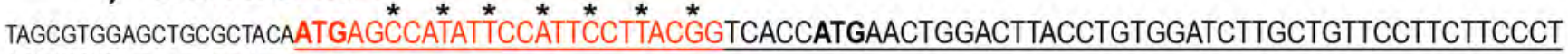
ACACAGGTCAGACAAGGGGCTGGT

\section{2) MO-splice-kit-S}

GAGAGGCCGTTCCTAAAATCAATAACGGAGACGACAGAGTCACTGTTAATGTTGGTGAGCGATTTTCATTGGTATGCAGAGATGTTCA GTTAGTTAAATTGGCTTTTCAGAAGTTGGGCCCTCTGAAGAAACCTCGAGATTTGAAAAGCCGTTCATATAACAAAAGCAAAACTGATC AGATCATAGTCATTGCTAAAGCAGATCTCAAGCACGTCGGAAGATATATATGCACGAATACAGAGACACACGAAAATACCTCTGTCTCT CTCTTTGTAAAGGGTAAGTCATTCATAAGGTTACACCTCTAACCCT

\section{C}

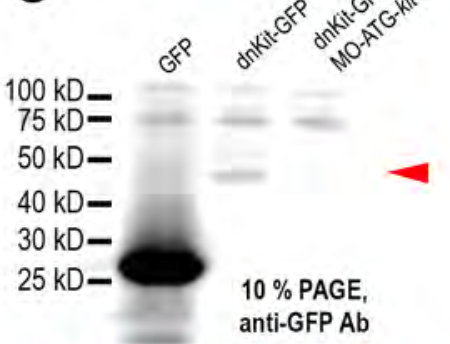

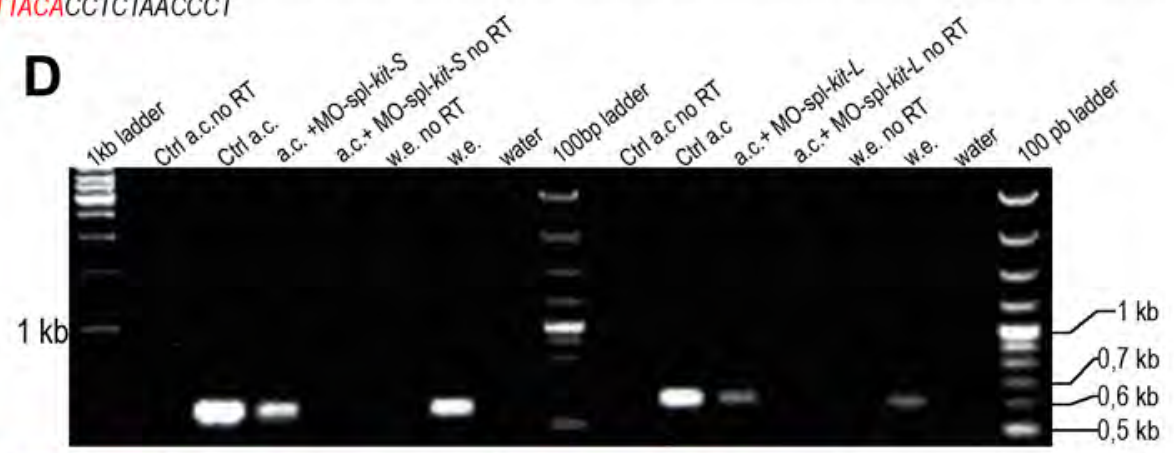
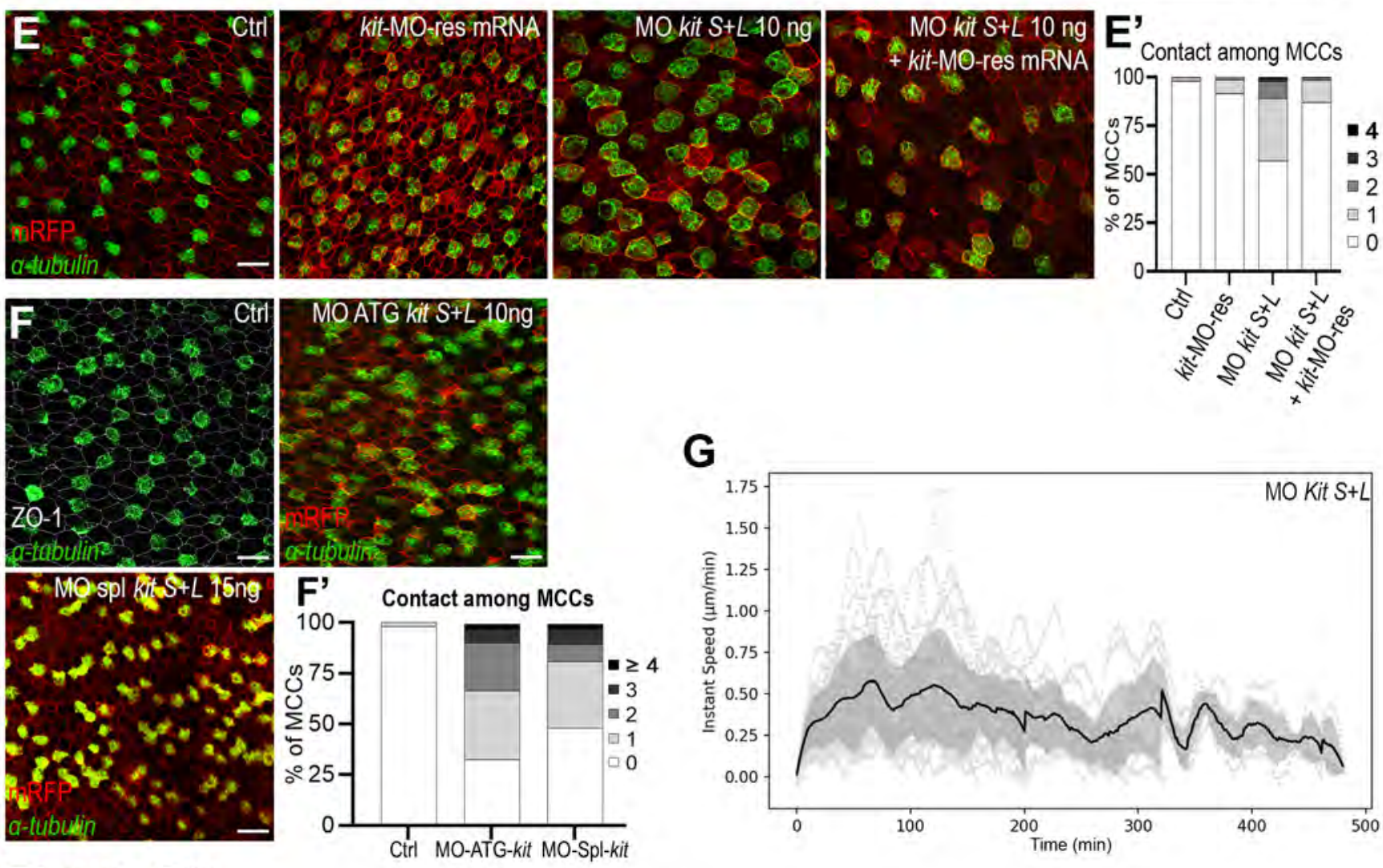

\section{Figure $\mathrm{S6}$}




\section{A scf-L}

1) GAAATGCACGGATTAGTTGAAATATGCTGGGGCTCCAGAGCAGCTAAGGGGATAATAGACAAGCTACCTAAATTGGATTGCTTTATCTTTTCCTTATGAAGAAGAC AAAAGTAAGTAAGATGGAATATTGTATGTCTCAAAAGAT

2) ACTTGGATTATCATATGCATTTATCTTCAACTGTTTCTCCATTGCTTTGGGAATCCATGTGGAAATCCAATCACTGATGCTGTAAATG ACATTCAGAAACTGGTAAGAGTTTTACCTAGCCAACCATTT

\section{B scf-S}

tTAGCTGAAATATGCTGGGGCTCCAGAGCAGCTAAGgGGATAATAGACAAGCTACCTGAATTGGATTGCTTTATCTTTTCCTTATGAAGAAGACAAAAA
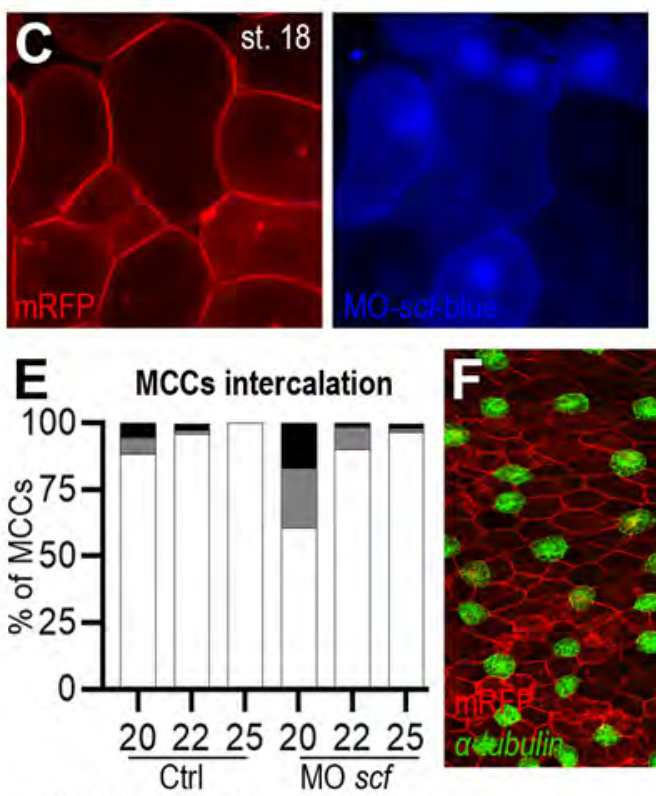

$\because$ nd $\square$ Non intercalated $\square$ Intercalated

$\mathbf{F}^{\prime}$

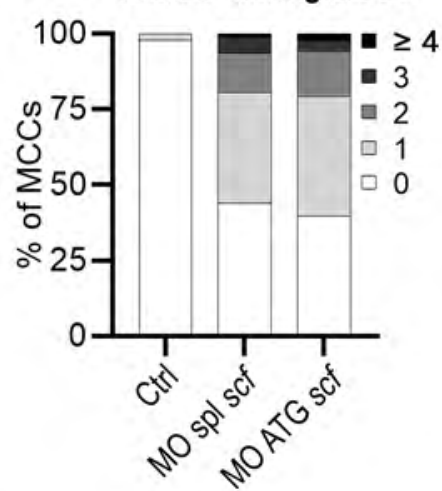

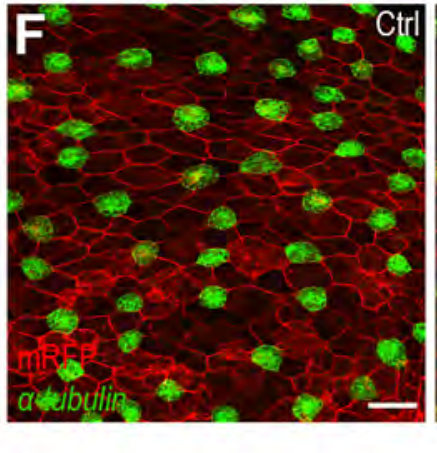
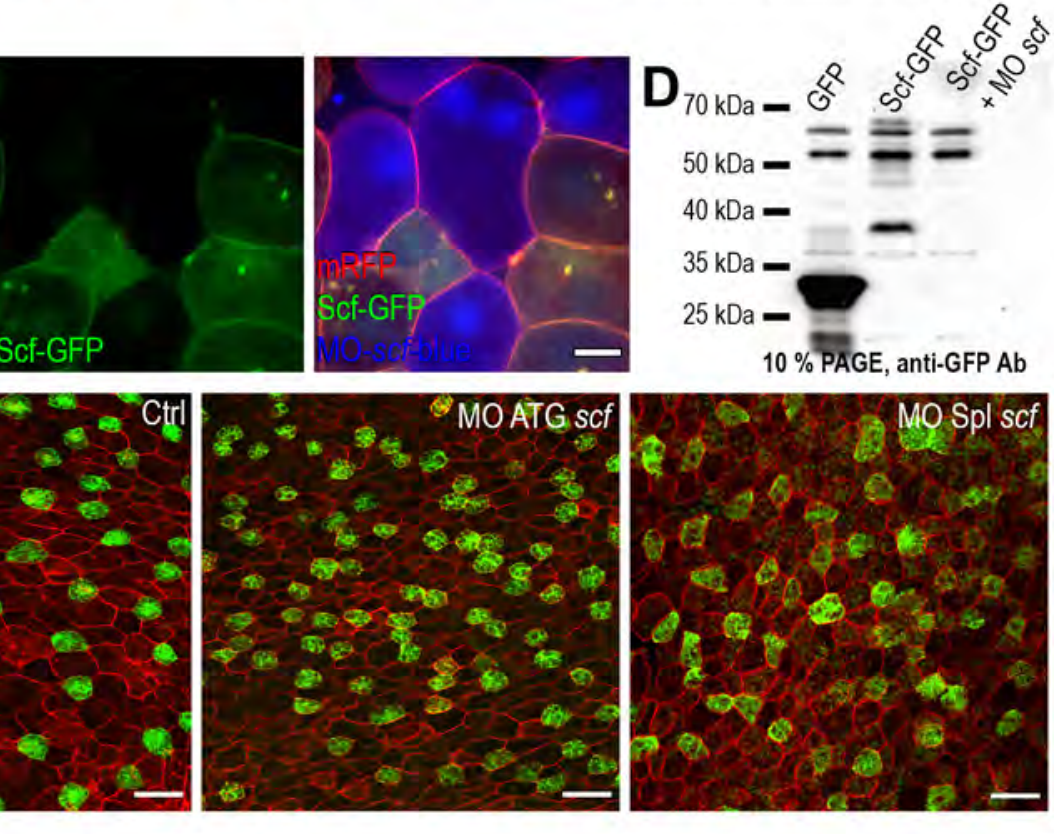

H
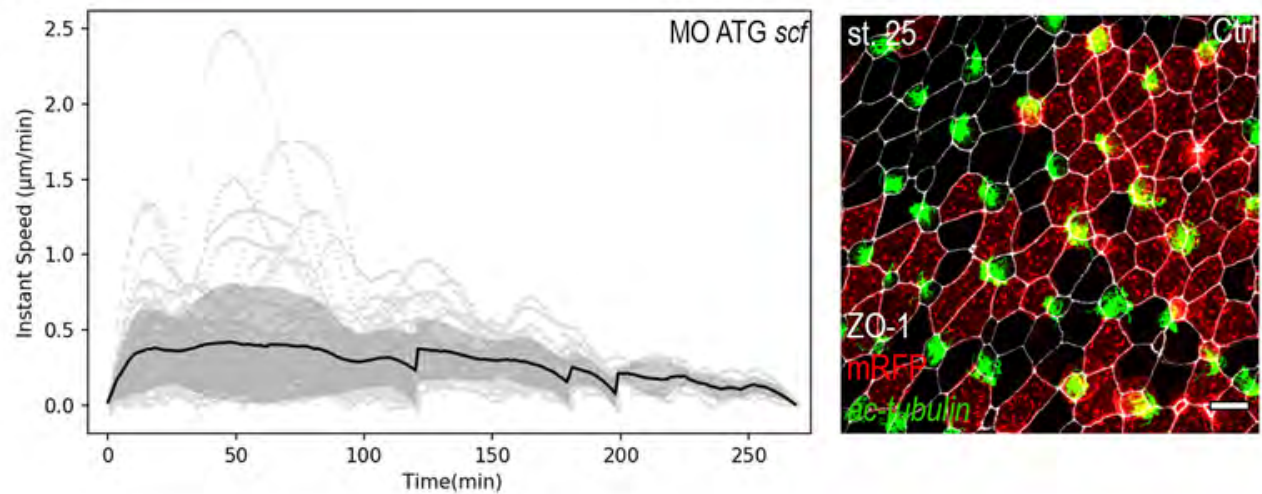

Figure S7 


\begin{tabular}{|c|c|c|c|c|}
\hline Figure & Stage/ condition & n cells & n embryos & n experiments \\
\hline \multirow{6}{*}{$1 \mathrm{~B}$} & 14 & 456 & 4 & 3 \\
\hline & 16 & 591 & 5 & 3 \\
\hline & 18 & 408 & 4 & 3 \\
\hline & 20 & 761 & 6 & 3 \\
\hline & 22 & 572 & 6 & 3 \\
\hline & 25 & 450 & 6 & 3 \\
\hline \multirow{4}{*}{$1 \mathrm{C}$} & 14 & 422 & 3 & 2 \\
\hline & 16 & 587 & 4 & 2 \\
\hline & 18 & 306 & 3 & 2 \\
\hline & 20 & 331 & 2 & 1 \\
\hline \multirow{6}{*}{$1 \mathrm{D}$} & 14 & 440 & 3 & 2 \\
\hline & 16 & 490 & 4 & 3 \\
\hline & 18 & 306 & 3 & 2 \\
\hline & 20 & 331 & 2 & 1 \\
\hline & 22 & 384 & 4 & 2 \\
\hline & 25 & 227 & 4 & 2 \\
\hline $2 B$ & Ctrl & 55 & 6 & 6 \\
\hline \multirow{2}{*}{$3 \mathrm{G}$} & Ctrl & 108 & 4 & 4 \\
\hline & CK-666 & 62 & 5 & 5 \\
\hline \multirow{3}{*}{$3 \mathrm{H}$} & Ctrl & 94 & 10 & 10 \\
\hline & Ctrl $\cup 90^{\circ}$ & 93 & 10 & 10 \\
\hline & CK-666 & 26 & 3 & 3 \\
\hline \multirow{3}{*}{31} & Ctrl & 227 & 4 & 4 \\
\hline & CK-666 & 209 & 2 & 2 \\
\hline & SMIFH2 & 144 & 2 & 2 \\
\hline \multirow{3}{*}{ 3J } & Ctrl & 248 & 4 & 1 \\
\hline & CK-666 & 491 & 6 & 1 \\
\hline & SMIFH2 & 457 & 6 & 1 \\
\hline \multirow{3}{*}{$5 \mathrm{C}$} & Ctrl & 108 & 4 & 4 \\
\hline & Axtitinib & 64 & 3 & 3 \\
\hline & MO kit & 147 & 5 & 5 \\
\hline \multirow{3}{*}{$5 C^{\prime}$} & Ctrl & 94 & 10 & 10 \\
\hline & Axtitinib & 56 & 9 & 9 \\
\hline & MO kit & 70 & 5 & 5 \\
\hline \multirow{9}{*}{$5 \mathrm{G}$} & Ctrl st 20 & 683 & 6 & 2 \\
\hline & Ctrl st 22 & 267 & 4 & 2 \\
\hline & Ctrl st 25 & 377 & 6 & 4 \\
\hline & Axtitinib st 20 & 441 & 5 & 2 \\
\hline & Axtitinib st 22 & 625 & 4 & 2 \\
\hline & Axtitinib st 25 & 724 & 6 & 3 \\
\hline & MO kit st 20 & 248 & 2 & 1 \\
\hline & MO kit st 22 & 370 & 3 & 1 \\
\hline & MO kit st 25 & 411 & 4 & 2 \\
\hline \multirow{9}{*}{$5 \mathrm{H}$} & Ctrl st 20 & 761 & 6 & 3 \\
\hline & Ctrl st 22 & 572 & 6 & 3 \\
\hline & Ctrl st 25 & 663 & 12 & 4 \\
\hline & Axtitinib st 20 & 830 & 8 & 3 \\
\hline & Axtitinib st 22 & 753 & 5 & 2 \\
\hline & Axtitinib st 25 & 735 & 8 & 2 \\
\hline & MO kit st 20 & 860 & 5 & 2 \\
\hline & MO kit st 22 & 998 & 8 & 2 \\
\hline & MO kit st 25 & 857 & 9 & 3 \\
\hline \multirow{3}{*}{51} & Ctrl & 227 & 4 & 4 \\
\hline & Axtitinib & 231 & 2 & 2 \\
\hline & MO kit & 219 & 2 & 2 \\
\hline
\end{tabular}

\begin{tabular}{|c|c|c|c|c|}
\hline Figure & Stage/ condition & $n$ cells & n embryos & n experiments \\
\hline \multirow{6}{*}{$6 \mathrm{C}$} & Ctrl st 20 & 761 & 6 & 3 \\
\hline & Ctrl st 22 & 572 & 6 & 3 \\
\hline & Ctrl st 25 & 518 & 8 & 4 \\
\hline & MO scf st 20 & 678 & 7 & 4 \\
\hline & MO scf st 22 & 545 & 6 & 1 \\
\hline & MO scf st 25 & 768 & 8 & 4 \\
\hline \multirow{2}{*}{$6 \mathrm{D}$} & Ctrl & 227 & 4 & 4 \\
\hline & MO scf & 220 & 3 & 3 \\
\hline \multirow{2}{*}{$6 \mathrm{~F}$} & Ctrl & 108 & 4 & 4 \\
\hline & MO scf & 64 & 5 & 5 \\
\hline \multirow{2}{*}{61} & Ctrl & 94 & 10 & 10 \\
\hline & MO scf & 62 & 5 & 5 \\
\hline \multirow{2}{*}{$7 \mathrm{~B}$} & Ctrl & 506 & 8 & 2 \\
\hline & paTub::Scf-GFP & 515 & 8 & 2 \\
\hline \multirow{4}{*}{ 7J } & Ctrl & 1589 & 16 & 2 \\
\hline & MO scf & 1031 & 8 & 2 \\
\hline & scf ECDw/oEx6 & 1261 & 10 & 2 \\
\hline & $s c f E C D w / o E x 6+\mathrm{MO} s c f$ & 1315 & 13 & 2 \\
\hline \multirow{3}{*}{$7 \mathrm{~N}$} & Ctrl & 1707 & 16 & 2 \\
\hline & kit mRNA 0,1 ng & 1526 & 15 & 2 \\
\hline & kit mRNA $1 \mathrm{ng}$ & 1704 & 17 & 2 \\
\hline \multirow{3}{*}{70} & Ctrl & 272 & 5 & 2 \\
\hline & kit mRNA 0,1 ng & 301 & 5 & 2 \\
\hline & kit mRNA $1 \mathrm{ng}$ & 299 & 5 & 2 \\
\hline \multirow{6}{*}{ S2 C } & Ctrl st 19 & 373 & 3 & 1 \\
\hline & CK-666 st 19 & 288 & 3 & 1 \\
\hline & SMIFH2 st 19 & 386 & 3 & 1 \\
\hline & Ctrl st 25 & 116 & 2 & 1 \\
\hline & CK-666 st 25 & 208 & 2 & 1 \\
\hline & SMIFH2 st 25 & 144 & 2 & 1 \\
\hline S2 & Ctrl & 55 & 6 & 6 \\
\hline E-G & CK-666 & 40 & 3 & 3 \\
\hline S4 & Ctrl & 55 & 6 & 6 \\
\hline$A, I, J$ & Axitinib & 67 & 5 & 5 \\
\hline \multirow{4}{*}{$\mathrm{S} 6 \mathrm{E}^{\prime}$} & Ctrl & 954 & 14 & 2 \\
\hline & kit-MO-Res & 837 & 13 & 2 \\
\hline & MO kit & 1049 & 14 & 2 \\
\hline & MO kit + kit-MO-res & 894 & 14 & 2 \\
\hline \multirow{3}{*}{ S6 F' } & Ctrl & 558 & 8 & 2 \\
\hline & MO kit ATG & 522 & 5 & 2 \\
\hline & MO kit spl & 592 & 6 & 2 \\
\hline S6G & MO kit ATG & 40 & 5 & 5 \\
\hline \multirow{6}{*}{ S7 E } & Ctrl st 20 & 683 & 6 & 2 \\
\hline & Ctrl st 22 & 267 & 4 & 2 \\
\hline & Ctrl st 25 & 226 & 4 & 2 \\
\hline & MO scf st 20 & 720 & 7 & 3 \\
\hline & MO scf st 22 & 408 & 4 & 1 \\
\hline & MO scf st 25 & 367 & 5 & 2 \\
\hline \multirow{3}{*}{ S7 F' } & Ctrl & 602 & 12 & 2 \\
\hline & MO Spl scf $20 \mathrm{ng}$ & 624 & 10 & 2 \\
\hline & MO ATG scf 10ng & 723 & 7 & 2 \\
\hline S7 G & MO ATG scf 10ng & 61 & 5 & 5 \\
\hline
\end{tabular}

Table S1: Total number of cells, embryos and repeat experiments for all graphs in all figures 